University of Tennessee Health Science Center UTHSC Digital Commons

\title{
Mapping of Cortical Motor Reorganization in Spinal Cord Injury
}

Leonid Rozhkov

University of Tennessee Health Science Center

Follow this and additional works at: https://dc.uthsc.edu/dissertations

Part of the Investigative Techniques Commons

\section{Recommended Citation}

Rozhkov, Leonid, "Mapping of Cortical Motor Reorganization in Spinal Cord Injury" (2001). Theses and Dissertations (ETD). Paper 224. http://dx.doi.org/10.21007/etd.cghs.2001.0270.

This Thesis is brought to you for free and open access by the College of Graduate Health Sciences at UTHSC Digital Commons. It has been accepted for inclusion in Theses and Dissertations (ETD) by an authorized administrator of UTHSC Digital Commons. For more information, please contact jwelch30@uthsc.edu. 


\title{
Mapping of Cortical Motor Reorganization in Spinal Cord Injury
}

\begin{abstract}
The annual incidence of spinal cord injury (SCI), not including those who die at the scene of the accident, is approximately 10,000 new cases in the United States. SCI, in its best outcome, may partially and temporarily disconnect the spinal cord from the brain. Some neuronal pathways remain intact in most $S$ $\mathrm{Cl}$ individuals, whose recovery depends on the utilization of the surviving connections. There is a change in the control of voluntary movements of the extremities by the cerebral cortex of the brain following spinal cord injury.
\end{abstract}

The technology of high-resolution EEG co-registered with MRI was applied to non-invasively investigate the brain's movement control network in both $\mathrm{SCl}$ and normal subjects. A series of active and passive movement tests were carried out to explore the changes that o ccur in the brain's cortical motor control after SCl. The spatial location of the brain areas active during motor tasks was identified in each individual and a statistical analysis was performed. It was found that activation of the motor cortex in SC I patients originated from a posterior part of the brain compared to the normal controls. The spatial difference was found to be statistically significant in the two groups with the p-values less than 0.05 in both active and passive movement tests.

We trust this study will contribute to the understanding of how the brain reorganizes its motor pathways after SCl. The clinical goal is the maximum utilization of the surviving connections to improve patient recovery. Also, understanding the neurona I activity and its topography in the brain is important in view of recent advances in experiments on primates. EEG can serve as an interface between the brain and computer-driven prostheses.

\section{Document Type}

Thesis

Degree Name

Master of Science (MS)

Program

Biomedical Engineering

Research Advisor

Lawrence Jordan, Ph.D.

\section{Keywords}

EEG, MRI, spinal cord injury, motor potential, primary motor area, neuroplasticity, source analysis, t-test

\section{Subject Categories}

Analytical, Diagnostic and Therapeutic Techniques and Equipment | Investigative Techniques | Medicine and Health Sciences 


\title{
MAPPING OF CORTICAL MOTOR REORGANIZATION IN SPINAL CORD INJURY
}

\author{
A Thesis \\ Presented for \\ The Graduate Studies Council \\ The University of Tennessee \\ Health Science Center
}

\author{
In Partial Fulfillment \\ Of the Requirements for the Degree \\ Master of Science \\ In the Joint Graduate Program in Biomedical Engineering \\ From The University of Tennessee \\ and \\ The University of Memphis
}

By

\section{Leonid I. Rozhkov}

December 2001 
Copyright $($ Leonid Rozhkov, 2001

All rights reserved 


\section{DEDICATION}

This thesis is dedicated to my parents

Mr. Igor Rozhkov

and

Mrs. Galina Rozhkova

for their unconditional love, support, and encouragement 


\section{ACKNOWLEDGEMENTS}

I would like to thank Dr. Joseph Green for his guidance and patience throughout the period of my work on his research project. I would like to gratefully acknowledge the enthusiastic supervision of Dr. Lawrence Jordan during this work. I would like to express my appreciation to my other committee members, Dr. Jack Buchanan and Dr. Paul Herron for their critical comments and assistance. I would also like to thank Dr. Frank DiBianca for his educational encouragement and helpful discussions. My thanks also to my colleagues on the project, Nancy Garrott, Peter St. Arnold and Darren Strother, for their being a true team, which I am proud to be part of. Finally, I would like to express my deepest gratitude and appreciation to my parents, Igor and Galina Rozhkov, who have always had the strongest belief in me and gave me their deepest love and emotional support, whatever may come. 


\section{ABSTRACT}

The annual incidence of spinal cord injury (SCI), not including those who die at the scene of the accident, is approximately 10,000 new cases in the United States. SCI, in its best outcome, may partially and temporarily disconnect the spinal cord from the brain. Some neuronal pathways remain intact in most SCI individuals, whose recovery depends on the utilization of the surviving connections. There is a change in the control of voluntary movements of the extremities by the cerebral cortex of the brain following spinal cord injury.

The technology of high-resolution EEG co-registered with MRI was applied to non-invasively investigate the brain's movement control network in both SCI and normal subjects. A series of active and passive movement tests were carried out to explore the changes that occur in the brain's cortical motor control after SCI. The spatial location of the brain areas active during motor tasks was identified in each individual and a statistical analysis was performed. It was found that activation of the motor cortex in SCI patients originated from a posterior part of the brain compared to the normal controls. The spatial difference was found to be statistically significant in the two groups with the $p$-values less than 0.05 in both active and passive movement tests.

We trust this study will contribute to the understanding of how the brain reorganizes its motor pathways after SCI. The clinical goal is the maximum utilization of the surviving connections to improve patient recovery. Also, understanding the neuronal activity and its topography in the brain is important in view of recent advances in experiments on primates. EEG can serve as an interface between the brain and computer-driven prostheses. 


\section{TABLE OF CONTENTS}

CHAPTER 1. PROJECT BACKGROUND

1.1.Introduction

1.2.Spinal Cord Injury: Statement of the Problem 2

1.3.Spinal Cord Injury: Neuroplasticity 3

1.3.1. General anatomy and physiology of the spinal cord and brain 3

1.3.2. Representation of voluntary movements in the cortex 5

1.3.3. Reorganization of cortical activity after spinal cord injury 8

1.4.Prospective Use in Rehabilitation $\quad 9$

1.5.Objectives of the Project 10

CHAPTER 2. METHODS 11

2.1.Introduction 11

2.2.Evoked Potentials with Voluntary and Passive Movements 11

2.3.Methods of Data Acquisition and Analysis 22

2.3.1. Subjects 22

2.3.2. Data acquisition 22

2.3.3. Triggering in passive movement tests 25

2.3.4. Analysis of physiological data 26

2.3.5. Dipole source analysis 29

2.4.Biophysical Aspects of EEG and Source Reconstruction 29

2.5. Calculation of the Coordinates of the Sites of Activation 41

2.6. Statistical Analysis: Two-Sample $t$-Test for Independent Samples 43

2.6.1. Equal variances case 44

2.6.2. Unequal variances case 46

2.6.2.1. The $F$ distribution $\quad 46$

2.6.2.2. The $F$ test 47

2.6.2.3. Two-sample $t$-test for independent samples with unequal variances $\quad 47$

CHAPTER 3. RESULTS AND CONCLUSIONS 50

3.1.Introduction $\quad 50$

3.2. Statistical Comparison of the Sites of Cortical Activation $\quad 50$

3.2.1. Motor potential distribution: the $F$ test 61

3.2.2. Motor potential distribution: the $t$-tests 61

3.3.Source Localization Results $\quad 64$

3.3.1. Results of the $F$ test 71

3.3.2. Source localization: the $t$-test 83

3.4.Discussion and Conclusion $\quad 83$

LIST OF REFERENCES

APPENDIX $\quad 91$

VITA $\quad 96$ 


\section{LIST OF TABLES}

Table 3.1. Control subjects: Normalized coordinates and angle values

in the motor potential localization study.

Table 3.2. Paraplegic patients: Normalized coordinates and angle values in the motor potential localization study.

Table 3.3. Tetraplegic patients: Normalized coordinates and angle values in the motor potential localization study.

Table 3.4. SCI patients: Normalized coordinates and angle values in the motor potential localization study.

Table 3.5. Controls and paraplegics: $F$ test for the equality of two variances.

Table 3.6. Controls and tetraplegics: $F$ test for the equality of two variances.

Table 3.7. Controls and SCI patients: $F$ test for the equality of two variances.

Table 3.8. $F$ test for the equality of two variances: MP localization.

Table 3.9. Controls and paraplegic patients: $t$-test for the equality of means.

Table 3.10. Controls and tetraplegic patients: $t$-test for the equality of means.

Table 3.11. Controls and SCI patients: $t$-test for the equality of means.

Table 3.12. $t$-test for the equality of means: MP localization.

Table 3.13. Control subjects: Normalized coordinates and angle values in the source localization study.

Table 3.14. SCI patients: Normalized coordinates and angle values in the source localization study.

Table 3.15. Controls and SCI patients: $F$ test for the equality of two variances in source localization study.

Table 3.16. Controls and SCI patients: $t$-test for the equality of means in source localization study. 


\section{LIST OF FIGURES}

Figure 1.1. Vertebral column and spinal nerves 5

Figure 2.1. Morphology of cortical potentials associated with voluntary $\begin{array}{ll}\text { right finger movement. } & 13\end{array}$

Figure 2.2. Results from the motor task.

$\begin{array}{ll}\text { Figure 2.3. Results from the sensory task. } & 17\end{array}$

Figure 2.4. 121-electrode EEG cap layout. $\quad 24$

Figure 2.5. Back of the DC amplifier system stimulus connector. 26

Figure 2.6. An electric diagram of the triggering device for passive movement tests. $\quad 27$

Figure 2.7. The triggering device for use in passive movement tests. 28

Figure 2.8. Single cell in a volume conductor of infinite extent. 35

Figure 2.9. A head model rendered from MRI images with EEG electrodes. 42

Figure 2.10. Strategy for testing for the equality of means in two independent, normally distributed samples. 49

Figure 3.1. An averaged epoch with a motor potential map in a paraplegic patient. $\quad 51$

Figure 3.2. An averaged epoch with a motor potential map in a normal control subject. 52

Figure 3.3. A 121-electrode cap layout with averaged epochs. 54

Figure 3.4. Mapping of motor potentials in left and right finger self-paced movements. 59

Figure 3.5. Mapping of motor potentials in left and right finger passive movements. $\quad 60$

Figure 3.6. Sectional views of the source localization results in a control subject, active movement test.

Figure 3.7. Source localization results with a segmented brain model - control subject, active movement test.

Figure 3.8. Sectional views of the source localization results in a control subject, passive movement test.

Figure 3.9. Source localization results with a segmented brain model - control subject, passive movement test.

Figure 3.10. Sectional views of the source localization results in a paraplegic patient, active movement test. 
Figure 3.11. Source localization results with a segmented brain model - paraplegic patient, active movement test.

Figure 3.12. Sectional views of the source localization results in a paraplegic patient, passive movement test.

Figure 3.13. Source localization results with a segmented brain model - paraplegic patient, passive movement test.

Figure 3.14. Dipole source localization in left and right finger self-paced movements. $\quad 80$

Figure 3.15. Dipole source localization in left and right finger passive movements. 


\section{LIST OF ABBREVIATIONS}

BOLD - blood oxygenation level dependent

BP - Bereitschaftspotential

CBF - cerebral blood flow

CNS - central nervous system

CSF - cerebrospinal fluid

DSA - dipole source analysis

EEG - electroencephalography

EMG - electromyography

ERP - event-related potential

fMRI - functional magnetic resonance imaging

fpMP - frontal peak of motor potential

GND - ground electrode

isMP - initial slope of motor potential

LFPM - left finger passive movement test

LFSP - left finger self paced movement test

M1 - primary motor cortex

MEG - magnetoencephalography

$\mathrm{MP}-$ motor potential

$\mathrm{MR}$ - magnetic resonance

MRCP - movement-related cortical potentials

MRI - magnetic resonance imaging 

NS' - negative slope
PET - positron emission tomography
pNS' - peak of the negative slope
ppMP - parietal peak of motor potential
rCBF - regional cerebral blood flow
RFPM - right finger passive movement test
RFSP - right finger self paced movement test
S1 - primary sensory cortex
SCI - spinal cord injury
SMA - supplementary motor area
SNR - signal-to-noise ratio
TTL - transistor-transistor logic 


\section{CHAPTER 1. PROJECT BACKGROUND}

\subsection{Introduction}

It is estimated that the annual incidence of spinal cord injury (SCI), not including those who die at the scene of the accident, is approximately 40 cases per million population in the U.S., or approximately 10,000 new cases each year. The number of people in the United States who are alive today and who have SCI has been estimated to be between 721 and 906 per million population, which corresponds to between 183,000 and 230,000 persons [1].

More than half (55\%) of the SCI population was injured between the ages of 16 and 30; the average age of injury is 31 years. $80 \%$ of SCI individuals are male and approximately half (46.5\%) are married at time of injury. The majority (90\%) of SCI individuals survive and live near-normal life spans. Initial hospitalization (an average of 15 days in acute care, then 44 days in rehabilitation), adaptive equipment and home modification costs following injury average $\$ 140,000$. Additional lifetime costs incurred by SCI individuals average $\$ 400,000$ and can reach as high at $\$ 1.35$ million depending on the severity of injury and the age at which injury occurred [1].

By developing therapies for those who are already spinal cord injured, and preventing new injuries, the United States would save as much as $\$ 400$ billion on future direct healthcare costs and additional indirect lifetime costs [1]. This has led to extensive research in studying motor functions of the brain and developing methods and devices to be used in rehabilitation of SCI patients. 
In this thesis, evoked response potentials were used to map motor activity of the brain in SCI patients and control subjects and a statistical analysis of the data was performed. A triggering device that helps establish the exact timing in certain evoked potential tests has been developed and experimentally explored.

\subsection{Spinal Cord Injury: Statement of the Problem}

The spinal cord may be damaged by compression from a variety of causes. These include tumors within or adjacent to the spinal cord, herniated intervertebral discs, blood clots, penetrating wounds caused by projectiles. Traumatic events include motor vehicle accidents (37\%), violence $(26 \%)$, falls $(22 \%)$, diving and sports injuries $(7 \%)$, or work-related or other accidents (the remaining 8\%) [1]. Paralysis may occur, depending on the location and extent of the injury.

Paralysis is the loss of voluntary motor function resulting from damage to nervous or muscle tissue. It may be classified as: paraplegia which affects both lower limbs, and tetraplegia, involving all four limbs. Paralysis may be complete or incomplete. Persons with tetraplegia have sustained injuries in the cervical portion of the spinal cord; those with paraplegia have damage in the thoracic, lumbar, or sacral regions of the spinal cord.

Since 1990 the most frequent neurologic category in the SCI population is incomplete tetraplegia (29.5\%), followed by complete paraplegia $(27.9 \%)$, incomplete paraplegia $(21.3 \%)$, and complete tetraplegia (18.5\%) [1]. Trends over time indicate an increasing proportion of persons with incomplete paraplegia and a decreasing proportion of persons with complete tetraplegia. 


\subsection{Spinal Cord Injury: Neuroplasticity}

Before discussing the mechanism of neuroplasticity and how the brain reorganizes itself after spinal cord injury, it is important to review first the general anatomy and physiology of the spinal cord and brain.

\subsubsection{General anatomy and physiology of the spinal cord and brain ${ }^{\dagger}$}

The spinal cord is protected by the vertebral column, meninges, cerebrospinal fluid (CSF), and vertebral ligaments. The meninges are three coverings that run continuously around the spinal cord and brain: dura mater, arachnoid, and pia mater. The spinal cord begins as a continuation of the medulla oblongata and ends at about the second lumbar vertebra in an adult. It contains cervical and lumbar enlargements that serve as points of origin for nerves to the limbs. The tapered inferior portion of the spinal cord is the conus medullaris, from which arise the cauda equina and filum terminale. The spinal cord is partially divided into right and left sides by the anterior median fissure and posterior median sulcus. The gray matter in the spinal cord is divided into horns and the white matter into columns. In the center of the spinal cord is the central canal, which runs the length of the spinal cord.

Parts of the spinal cord which can be observed in transverse section are the gray commissure; central canal; anterior, posterior, and lateral gray horns; and anterior, posterior, and lateral white columns which contain ascending and descending tracts. The spinal cord conveys sensory and motor information by way of the ascending and descending tracts, respectively. A

${ }^{\dagger}$ Material presented in this subdivision was substantially derived from Tortora GJ, Grabowski SR. Principles of anatomy and physiology. $8^{\text {th }}$ ed. New York, NY: HarperCollins Publishers Inc., 1996. 
major function of the spinal cord is to propagate nerve impulses from the periphery to the brain (sensory tracts) and to conduct motor impulses from the brain to the periphery (motor tracts). Sensory information travels along two main routes: the posterior column tract and the spinothalamic tracts. Motor information travels along two main routes: direct (pyramidal) tracts and indirect (extrapyramidal) tracts. Another function of the spinal cord is to serve as an integrating center for spinal reflexes. This occurs in the gray matter. A reflex is a fast, predictable, automatic response to changes in the environment that helps maintain homeostasis. Reflexes can be divided into spinal and cranial reflexes, depending on where the integrating center is located, or into somatic and autonomic (visceral) reflexes, depending on whether they involve contraction of skeletal muscles or adjust the activities of smooth muscles, the cardiac muscle, and glands.

The 31 pairs of spinal nerves are named and numbered according to the region and level of the spinal cord from which they emerge, as seen in Figure 1.1. There are 8 pairs of cervical, 12 pairs of thoracic, 5 pairs of lumbar, 5 pairs of sacral, and 1 pair of coccygeal nerves (not shown in the figure). Spinal nerves typically are attached to the spinal cord by a posterior root and an anterior root. All spinal nerves contain both sensory and motor axons (mixed). Branches of a spinal nerve include the dorsal ramus, ventral ramus, meningeal branch, and rami communicantes.

The principal parts of the brain are the brain stem, cerebellum, diencephalons, and cerebrum. The brain is protected by cranial bones, meninges, and cerebrospinal fluid. The cranial meninges are continuous with the spinal meninges and are named dura mater, arachnoid, and pia mater. Cerebrospinal fluid is formed in the choroid plexuses and circulates through the ventricles, subarachnoid space, and central canal. Most of the fluid is absorbed by the arachnoid 
villi of the superior sagittal blood sinus. Cerebrospinal fluid provides mechanical protection, chemical protection, and circulation.

The cerebrum is the largest part of the brain. Its cortex contains gyri, fissures, and sulci. The cerebral lobes are frontal, parietal, temporal, and occipital. The white matter is deep to the cortex and consists of myelinated and unmyelinated axons extending to other regions as association, commissural, and projection fibers. The sensory and motor areas are closely lined at the central sulcus. There are primary and secondary sensory and motor areas, including the supplementary motor area (SMA), premotor, etc. Association areas are concerned with more complex integrative functions.

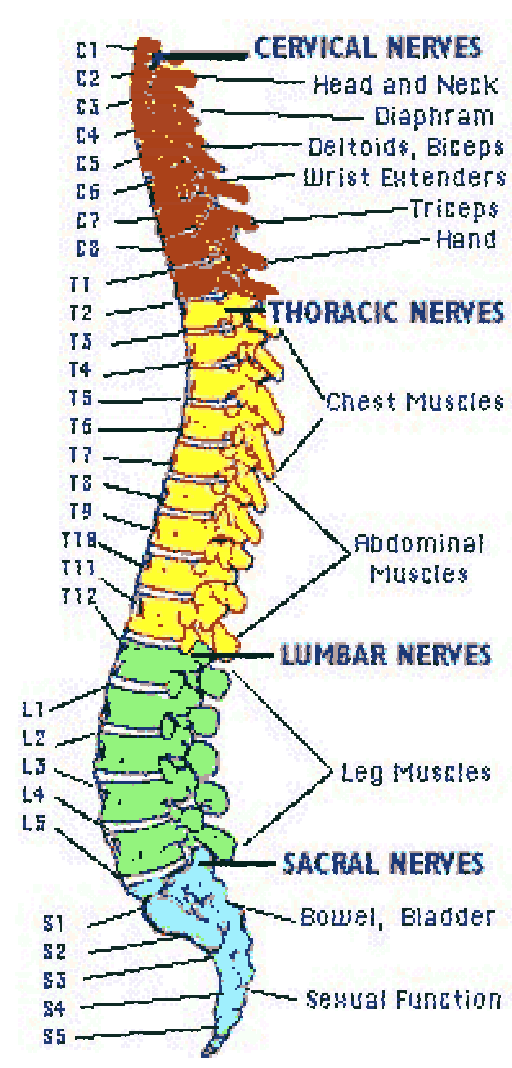

Figure 1.1. Vertebral column and spinal nerves

\subsubsection{Representation of voluntary movements in the cortex}

Sensory systems provide the input that informs the central nervous system (CNS) of changes in the external and internal environment. These are relayed to the motor network. As sensory information reaches the CNS, it becomes part of a large pool of sensory input. The incoming information is integrated with other information arriving from all other operating sensory receptors. The integration process occurs not just once but at many stations along the pathways of the CNS and at both conscious and subconscious levels. It occurs within the spinal 
cord, brain stem, cerebellum, basal ganglia, and cerebral cortex. As a result, a motor response to make a muscle contract or a gland secrete can be modified and responded to at any of these levels. The motor networks of the cerebral cortex play the major role for initiating and controlling precise, discrete muscular movements [2].

The most direct somatic motor pathways extend from the primary motor cortex (M1) into the spinal cord and out to skeletal muscles. Other pathways are less direct and include synapses in the basal ganglia, thalamus, reticular formation, and cerebellum.

The primary motor area is located in the precentral gyrus of the cerebral cortex and is the major control region for initiation of voluntary movements. The adjacent premotor area and the somatosensory area in the postcentral gyrus also contribute fibers to the descending motor pathways. As is true for the somatosensory area, different muscles are represented unequally in the primary motor area. The degree of representation is proportional to the number of motor units in a particular muscle of the body. For example, the muscles in the thumb, fingers, lips, tongue, and vocal cords have large representations while the trunk has a much smaller representation. Somatosensory and motor representations are similar but not identical for the same part of the body.

Nerve impulses for voluntary movements propagate from the motor cortex to somatic motor neurons that innervate skeletal muscles via the direct or pyramidal pathways. The simplest of these pathways consists of sets of two neurons, upper motor neurons and lower motor neurons. The axons of the upper motor neurons descend in the pyramidal tract to the spinal cord. Axons terminate in nuclei of cranial nerves or in the anterior gray horn of the spinal cord. Lower motor neurons extend from the motor nuclei of cranial nerves to skeletal muscles of the face and head and from the anterior horn of each spinal cord segment to skeletal muscle fibers of the trunk 
and limbs. Close to their termination point, most upper motor neurons synapse with an association neuron, which, in turn, synapses with a lower motor neuron. Upper motor neurons also synapse directly with lower motor neurons.

Recent developments in brain mapping have shed light on different topographic aspects of the human motor and sensory functions, especially those of the hand. Comparison of various brain mapping data, obtained from different modalities, requires a common anatomic coordinate. The central sulcus may be in many cases as such a reference structure. The central sulcus itself is a purely anatomic structure. It is a constant landmark, a generally continuous and sinuous deep sulcus on the convexity of the brain [3]. These features, however, become clear only after removal of the pia mater and vessels from a cadaver brain. Reconstructed surface-rendered MR images provide a good way to localize the central sulcus, as well as other structures, in living humans.

Nii et al. [4] performed experiments focused on studying the topography of hand areas, especially in relation to the position of the central sulcus. The goal was to evaluate the representation of the hand in the sensorimotor cortex. Hand responses were obtained in over thirty patients who underwent implantation of subdural grid electrodes for evaluation and surgical treatment. These responses were analyzed according to the presence of motor, sensory, and mixed motor and sensory responses. It has been shown that hand motor responses occurred not only in the precentral gyrus but also in the postcentral gyrus, with great variability in superior-inferior distribution. Sensory responses also occurred in both the precentral and postcentral gyri with a distribution more ventral than that of motor responses. Even in the Rolandic cortex, the same small area could contain both motor and sensory information and representations of different parts of the body. These data indicate a marked variability in the 
location of the human cortical hand area, and suggest that motor and sensory hand cortices overlap and are not divided in a simple manner by the central sulcus.

Duffau et al. [5] reported an observation of real-time intraoperative evidence of a retrocentral redistribution of motor areas with an unmasking of precentral redundant motor sites, before and after, respectively, surgical removal of a precentral arteriovenous malformation using direct electrical stimulation. That study showed large-scale plasticity of the motor function behind the central sulcus as the result of an arteriovenous malformation and the existence of redundant precentral and postcentral motor areas for the same movement, suggesting that the central sulcus does not simply divide motor and sensory functions.

\subsubsection{Reorganization of cortical activity after spinal cord injury}

Recovery from incomplete paraplegia or tetraplegia significantly depends on the preservation of neurons and the physiologic reorganization of the brain and spinal cord motor networks. The mechanism of cortical reorganization still remains unclear. It is believed that it may be possible to improve functional recovery by influencing reorganization once the underlying processes are better understood. Green et al. [6] used high-resolution electroencephalography (EEG) to investigate the effect of SCI on evoked cortical potentials associated with movements of the fingers and toes, called in the literature movement-related cortical potentials (MRCP). The motor potential (MP) of the MRCP was selected for mapping purposes because it had been localized to the primary motor area of the hemisphere contralateral to the movement [7]. Studies of Green et al. [6,8] have shown that motor potentials associated with finger and toe movements tend to relocate posteriorly in SCI patients as compared with 
those in normal control subjects. An explanation has been proposed, i.e. because of loss of axons in the primary motor area, contribution of the primary sensory area to the generation of the motor potential predominates, causing the origin of the potential to move behind the central sulcus. Since peak latencies of the motor potential are significantly longer than normal, this also supports a change in pathway. This suggestion has been based on the hypothesis that there is a relative sparing of the outflow of axons from the primary sensory area to the spinal cord after SCI. These axons have a more medial and posterior course, which may render them less vulnerable to trauma.

\subsection{Prospective Use in Rehabilitation}

Wessberg et al. [9] performed experiments on real-time prediction of hand trajectory by ensembles of cortical neurons in monkeys. He recorded the simultaneous activity of large populations of neurons, distributed in the premotor, primary motor and posterior parietal cortical areas, as two monkeys performed two distinct motor tasks. Cortically derived signals were used for the generation of one-dimensional and three-dimensional signals to control robot movements in real time. These findings support the notion that motor signals derived from ensembles of cortical neurons could be used for long-term control of prosthetic limb movements [10]. This research group has shown that chronically implanted microwires can yield reliable recordings in primates for at least twenty-four months. This suggests that a combination of denser multi-wire arrays with implantable integrated circuits, designed to handle all real-time signal processing and mathematical analysis, could one day form the basis of a brain-machine interface for allowing paralyzed patients to control voluntarily the movements of prosthetic limbs. 


\subsection{Objectives of the Project}

From the above discussion, it can be realized that an appropriate combination of functional and anatomical data acquisition methods can provide information for the estimation of spatio-temporal activity of the brain during various motor and sensory tasks.

A high-resolution electroencephalography technique is used in this project for the acquisition of functional data. A co-registration of anatomical head size along with the electrode positions on the scalp is also performed to calculate the spatial location of active areas in each individual. Magnetic resonance imaging (MRI) is used as a cooperative modality to derive putative current sources in the cortex responsible for the generation of the distribution of electrical potentials on the scalp. The source reconstruction study provides means of localizing active brain areas using individual anatomy.

The objectives of this research are as follows:

- Map motor-related cortical potentials associated with finger movements in SCI patients and control subjects.

- Carry out source localizations.

- Estimate the nature and extent of cortical motor reorganization after spinal cord injury. This project complements the study of Green [6,8]. It is aimed at obtaining results by engaging a larger population of subjects, differentiating SCI patients by their condition (paraplegic and tetraplegic patients) and performing statistical analysis of data from both motor potential and source localization studies. 


\section{CHAPTER 2. METHODS}

\subsection{Introduction}

Theories and methods for studying motor activity of the brain by using high-resolution electroencephalography are detailed in this chapter. First, the state of the art is described by analyzing methods and accomplishments of various research groups who have made contributions to our present understanding of evoked potentials with voluntary and passive movements. Then, the methods used in this project are presented, followed by a discussion of the underlying theory of the method called "dipole source analysis". At the end of the chapter, the algorithm for calculation of the coordinates of the sites of activation in motor tasks is described, along with statistical analysis of the data using two-sample $t$-test for independent samples.

\subsection{Evoked Potentials with Voluntary and Passive Movements}

Knowledge of the regions of the brain that are active in voluntary movements and of the timing of their activation with respect to the movement onset is clearly important in understanding the physiology of voluntary movements in humans. Studies using positron emission tomography (PET) and functional magnetic resonance imaging (fMRI) have identified the probable brain regions engaged in the execution of voluntary movements, but they tell us 
little about the timing of cortical activation. Movement-related cortical potentials give detailed timing information [7].

The EEG potential related to the onset of self-paced voluntary movement has several components. The most studied component is the slowly rising bilateral negativity preceding the onset of voluntary movement, originally called the Bereitschaftspotential (BP) by Kornhuber and Deecke [11]. The negativity was observed to have two phases: an early, slowly rising phase, which begins at 1500-2000 ms before the movement onset and shows a symmetrical and widespread scalp distribution and a later, more rapidly rising phase which starts at 300-500 ms before the movement onset and is larger over the centro-parietal region contralateral to the movement. Some research groups have restricted the term BP to the early phase and have used the term negative slope (NS'), for the later phase. Figure 2.1 shows a simplified version of typical EEG waveforms associated with voluntary finger movements. The motor potential complex has several components. Tarkka and Hallett [7] investigated spatial distribution of scalp-recorded motor potentials. They identified four distinct negative events in the averaged EEGs of normal subjects in self-paced voluntary finger movements. These events were the peak of the negative slope (pNS'), the initial slope of motor potential (isMP), the parietal peak of motor potential (ppMP), and the frontal peak of motor potential (fpMP). NS' had a wide distribution, covering the parietal region with slight contralateral predominance. The isMP mapped focally to the contralateral hand motor area on the scalp. The location of ppMP was similar to that of isMP. It was proposed that the ppMP might represent the terminal phase of the motor cortex firing. Topographic mapping revealed the highest negativity on the scalp localized anterior and medial to motor cortex with a contralateral preponderance and possible location over the 


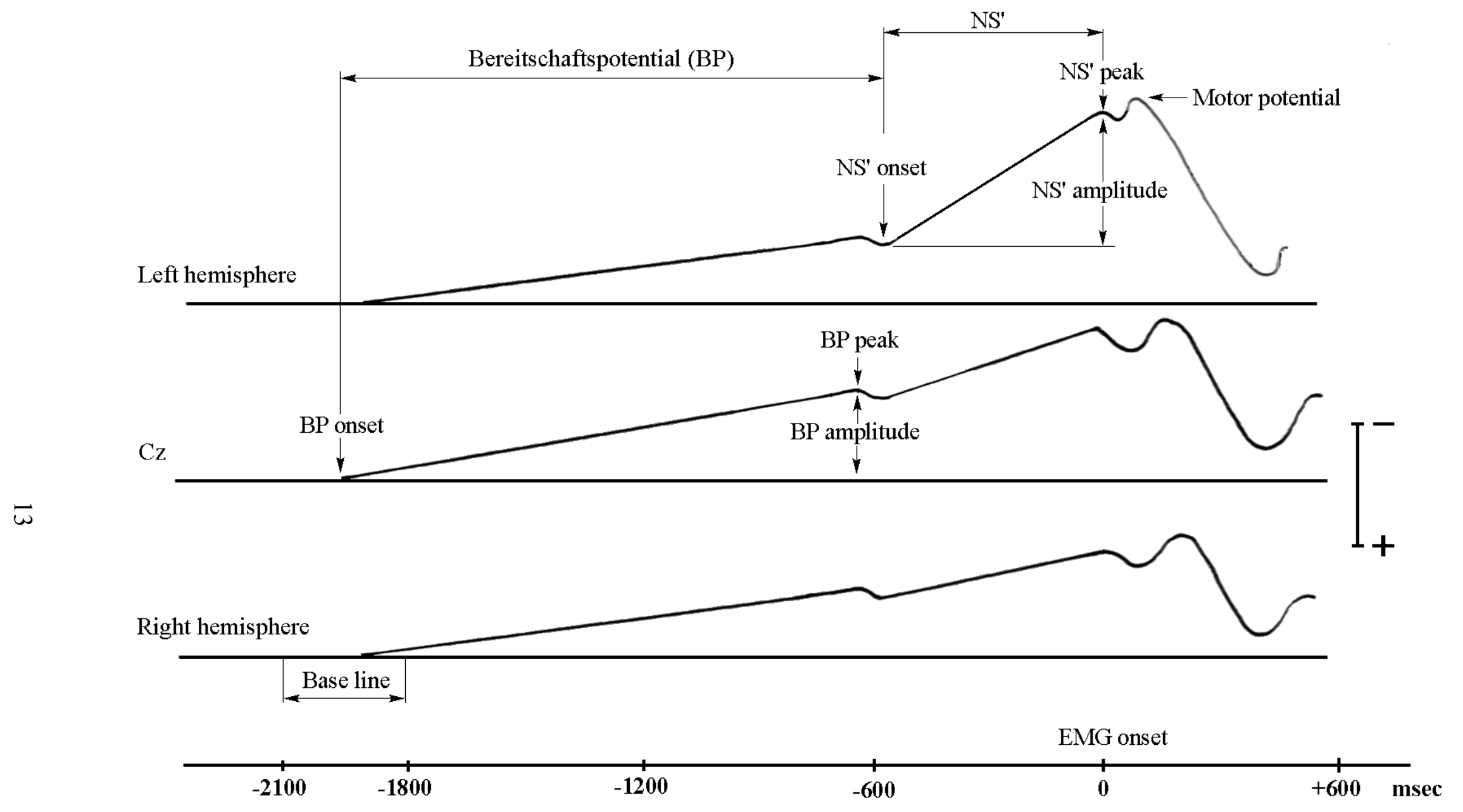

Figure 2.1. Morphology of cortical potentials associated with voluntary right finger movement.

Source: Kitamura J, Shibasaki H, Takagi A, Nabeshima H, Yamaguchi A. Enhanced negative slope of cortical potentials before sequential as compared with simultaneous extensions of two fingers. Electroencephalogr Clin Neurophysiol. 1993 Mar; 86(3):176-182. 
supplementary motor area. The final peak, the fpMP, corresponded to the peak of the motor potential in self-paced finger movement. The location of fpMP in that study showed that the peak negativity of the motor potential complex was not likely to be generated in the hand motor area, contrary to suggestions of Neshige et al. [12]. No negativity or positivity was observed preceding the movement onset in the recordings of passive finger movements [7]. After the movement onset, a sharp well-formed negativity appeared with a peak latency of about $100 \mathrm{~ms}$. The scalp topography of this potential revealed a slightly contralateral, anterior to motor cortex, peak negativity. The sensory feedback from the muscle, joint, and cutaneous afferents could be the major source of the fpMP. The localization of the fpMP in topographic mapping suggested involvement of the supplementary motor area. The termination of the entire negativity, which the fpMP represents, appeared after the onset of electromyographic (EMG) activity in both passive and voluntary movement conditions, and was located clearly in front of the primary motor cortex. These data show that sensory input from the periphery has an important role in producing the negativity after the onset of EMG activity. The SMA has been thought to be involved in the generation of the scalp-recorded fpMP.

Toro et al. [13] went forward and carried out source analysis of scalp-recorded movement-related electrical potentials. They were looking for equivalent current sources generating the movement-related cortical potentials at the instants of the peak of the negative slope and the frontal peak of the motor potential, using a spherical head model. The time interval for the analysis was $-200 \mathrm{~ms}$ to $200 \mathrm{~ms}$ with respect to the movement onset. The pNS' and the fpMP were chosen because they are the most distinct events of the movement-related cortical potentials immediately before and after the movement onset and, therefore, are believed to represent the points in time of maximal activation of the underlying cortical structures. While the 
early activity of the Bereitschaftspotential most likely reflects aspects of intention and preparation to move, components close to the movement onset are more likely related to the activation of cortical structures involved in the generation of descending corticospinal volleys and to the arrival of sensory feedback information related to the movement itself. Direct subdural recordings in humans [12] have shown that the motor potential (which is understood to have two parts with distinct topography and latency, namely the ppMP and the fpMP) has maximal amplitude over the contralateral primary sensorimotor cortex.

Recordings from the interhemispheric fissure in humans also show that the contralateral SMA generates a well-formed MP during simple, self-paced finger movements [12]. These studies suggest that the contralateral sensorimotor cortex and the SMA might contain the sources of the scalp-recorded motor potential. Dipole modeling showed that the motor potential arose from the combined activity of the contralateral primary sensorimotor region and the supplementary motor area. The activation of the SMA contributed to the origin of the motor potential in 6 out of 10 subjects studied. These results also agree in part with a magnetoencephalographic (MEG) study $[14,15]$ of the generators of activity at about $100 \mathrm{~ms}$. The magnetic counterpart to the fpMP, known as the movement-evoked magnetic field I, was modeled by a single generator in the contralateral sensorimotor cortex. MEG studies, however, have not shown evidence of SMA participation in the magnetic responses to self-paced voluntary movements, which may be due to the presence of bilateral SMA generators with exactly opposite orientation, resulting in field cancellation [14,15].

Functional MRI studies of Turner et al. [16] of motor and sensory tasks showed activations in primary motor and sensory cortex, respectively, in normal subjects and some variations in the locus of activity in SCI patients. In the motor task, both groups also showed a 
cluster of activity along the medial wall, in the supplementary motor area, Figure 2.2. It is worth noting that the sensory task was performed on the subjects by rubbing their palms with a wooden dowel, which means that no proprioceptive input was provided. Figure 2.3 demonstrates the results from the sensory task. It can be seen that no activity was detected in the SMA. Recalling the results obtained in a passive movement paradigm by Tarkka and Hallett [7] and comparing them with the results of the sensory tasks in this study, indicate that the SMA may become active in tasks with proprioceptive feedback.

Shibasaki et al. [17] compared the cortical potentials associated with a voluntary brisk finger movement with those evoked by a similar passive movement in order to clarify the functional significance of those components, especially the components following the movement. This work was one of the earliest done on passive movements and was aimed to investigate the scalp topography of the cerebral responses evoked by passive movements. To elicit passive movement, a soft hemp string was used. The string was bound around the distal portion of the middle phalanx of the middle finger and the other end of the string was pulled up briskly by the examiner. A trigger pulse was obtained using a photometer for both voluntary and passive movements. Results of the experiment on studying cortical potentials associated with passive middle finger extension showed that there was no significant consistent potential change before the movement onset in the individual records. Thus, with regard to averaged potentials before the onset of movement, there was a marked difference in the waveform between voluntary and passive movement, but after the onset of movement, certain common features were noted. It was pointed out that the absence of premotion components in passive movement confirmed that these potentials were related to voluntary movement as had been originally proposed by 

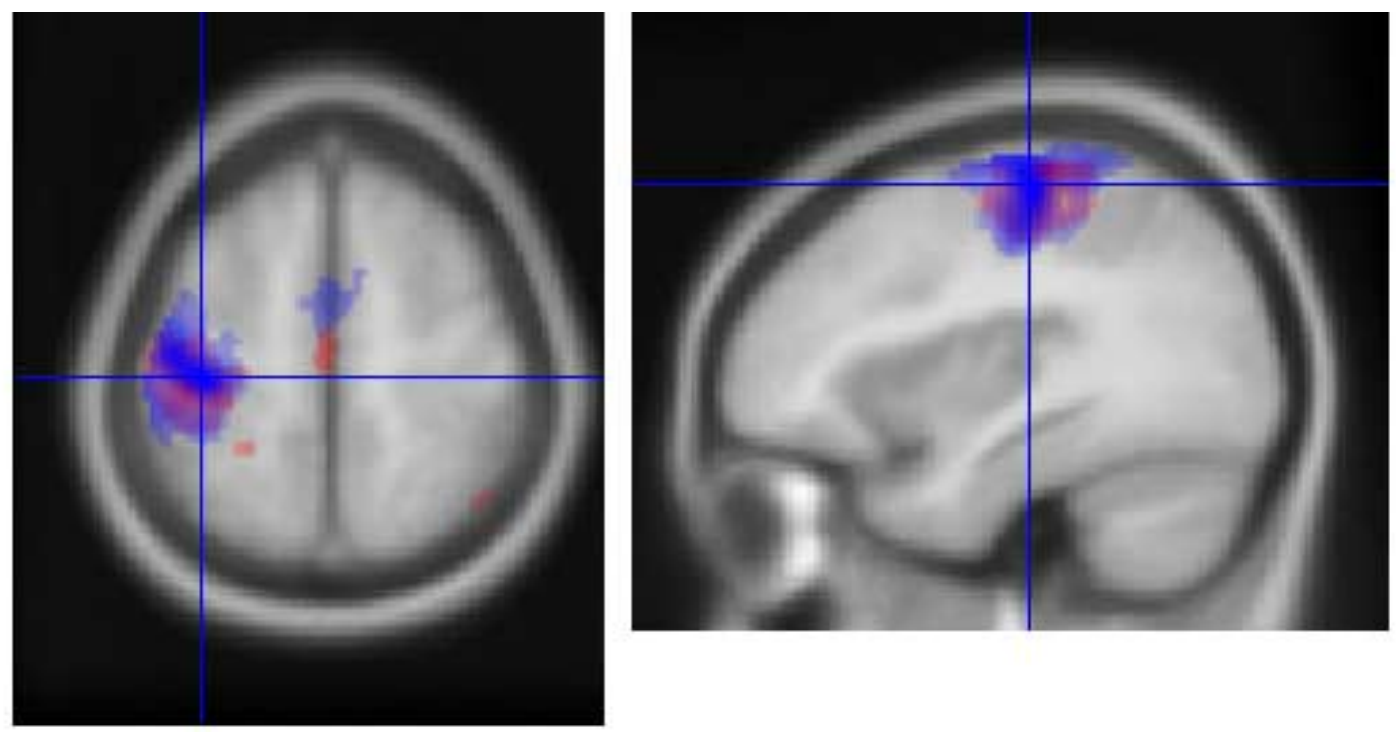

Figure 2.2. Results from the motor task. The SCI group data are in red and the control group data are in blue.
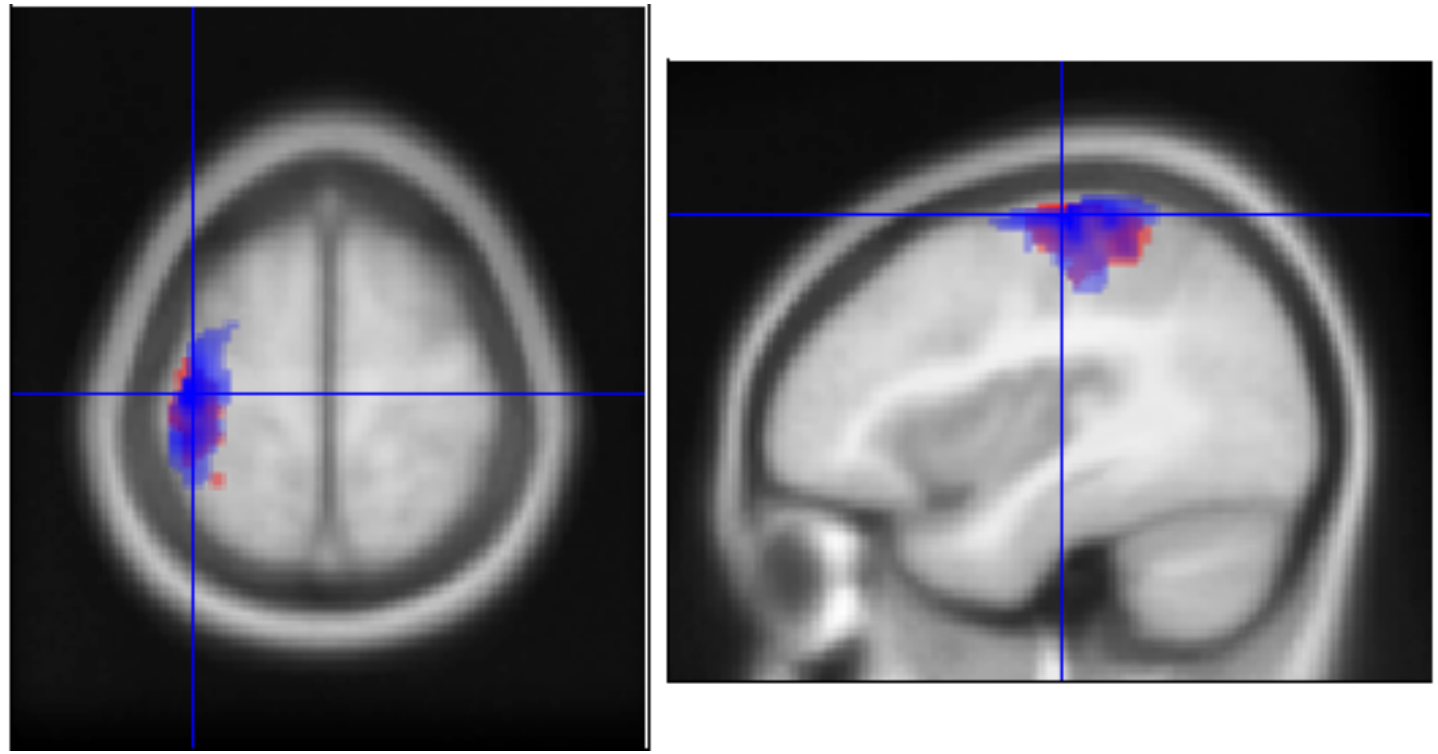

Figure 2.3. Results from the sensory task. The SCI group data are in red and the control group data are in blue.

Source: Turner JA, Lee JS, Martinez O, Medlin AL, Schandler SL, Cohen MJ. Somatotopy of the motor cortex after long-term spinal cord injury or amputation. IEEE Trans Neural Syst Rehabil Eng. 2001 Jun; 9(2):154-160. 
Kornhuber and Deecke [11]. Several negative peaks were identified in the passive movementevoked potential. A sharp negative peak occurred over the contralateral precentral region $16 \mathrm{~ms}$ after the photometer trigger. It was postulated that these components might be the projected potential fields from a dipole source within the central sulcus and might represent a kinesthetic feedback from the muscle afferents.

Weiller et al. [18] studied brain representation of passive and active movements using positron emission tomography. Some of the objectives were to segregate the efferent motor from the afferent sensory component of a voluntary movement, and to study the brain representation of passive movement itself. Right elbow flexion-extension movements were performed using a torque motor during the passive condition. To exclude any possible voluntary movements during the passive condition, muscle activity was monitored by electromyographic surface electrodes on biceps and triceps muscles. It was observed that activation in contralateral sensorimotor cortex was almost identical in magnitude, extent, and location with both passive and active movements. However, the regional cerebral blood flow $(\mathrm{rCBF})$ in the somatosensory area was increased during active movement more than passive movement. The focus of maximum activation was also more inferior than during passive movements. Activation was not restricted to the postcentral gyrus but covered both pre- and postcentral gyrus. Brain structures processing afferent information were intermingled with those processing motor output in both pre and postcentral areas. There was also noted a tight anatomical coupling between somatosensory input and motor output in the primary cortices. Afferent input is carefully related to the motor cortical output of that same region. If a muscle is passively stretched, the afferent input from the muscle spindles projects to that area of the cortex which can excite cells producing contraction of the same muscle. 
The SMA was also activated during both active and passive movements. The rCBF increase, however, was reported to be significantly stronger during active movements and was located more inferiorly. These data support the idea of a segregation of motor functions within the medial wall of the hemispheres. Passive movements may be useful in the study of the reorganization of the brain in patients who are unable to move their extremities.

In another work of the same research group under Weiller [19], passive movement paradigm was used to investigate whether cortical reorganization was related to the recovery of lost function in stroke patients. The following important results were obtained:

- The patients with little cortical activation, restricted to the motor cortex in the damaged hemisphere initially before recovery had started, showed no recovery after three months.

- All patients with good or excellent recovery after three months showed extensive reorganization at the initial scan, due to activation of the ipsilateral motor cortex. It was stressed that passive movement representing proprioceptive feedback in stroke patients before recovery elicited many of the reorganizational changes found during active movement after recovery. Sensory reorganization precedes motor reorganization, and therefore may trigger motor reorganization. The assessment of sensory reorganization early may indicate the potential for motor reorganization and be a useful prognostic tool.

Alary et al. [20] studied event-related potentials (ERP) elicited by passive movements using functional magnetic resonance imaging. His experiment was designed to produce repetitive passive extensions of right and left forefingers to induce propriostimulation (muscle and articular receptors). Skin receptors were also involved in the paradigm. Extension of the subject's forefinger was obtained by passive mobilization applied manually by an operator at a slow and random rate. There was, however, a surprising fact in the design of the experiment: an EMG 
activity of flexor digitorum sublimus was observed after the beginning of the movement, which was interpreted as a stretch reflex activity. The EMG was also used to monitor muscular relaxation during the whole procedure. In the data processing part of the experiment, the onset of flexor digitorum sublimus EMG was used as trigger for the epoching process. Results of the experiment suggest that proprioceptive inputs follow a sequential processing through the contralateral rolandic area and then simultaneously in the supplementary motor area and in inferior parietal lobe.

Nelles et al. [21] studied functional reorganization after hemiplegic stroke by using passive elbow movements as an activation paradigm with positron emission tomography. In the design of the experiment, the plegic arm of patients was abducted at the shoulder level to 70 degrees and placed in a forearm brace, which was connected to a torque motor. The torque motor generated passive flexion and extension of the elbow at an amplitude of 0 to 90 degrees and a frequency of $0.5 \mathrm{~Hz}$. In normal subjects, passive forearm movements produced maximum increases of $\mathrm{rCBF}$ in the contralateral sensorimotor cortex, supplementary motor area, and bilateral inferior parietal cortex. This pattern of activation corroborated earlier reports of a PET experiment using the same passive movement paradigm in a larger group of healthy individuals [18]. Also, Alary and colleagues [20] reported activation of similar anatomical areas by using fMRI during passive finger movements in normal subjects.

Mima et al. [22] conducted an experiment to identify the neural mechanisms associated with the "pure" motor aspects of the finger movement with minimal involvement of sensory afferent inputs by comparing cerebral blood flow (CBF) changes in an active volitional and passively caused movements. For the passive movement task, a specially equipped device for flexing the finger was utilized. Brisk passive movement of the same finger (20 degrees) was 
elicited every $0.5 \mathrm{~s}$ by a servomotor, driven to cause flexion of the finger. By comparing the CBF changes during voluntary movement and those during passive movement (and applying almost identical movement conditions), the results suggested that the supplementary motor areas were included only in the voluntary active aspects of movement and were not associated with sensory feedback inputs to any appreciable degree.

Turner et al. [16] performed an fMRI study to investigate hand representation in primary motor and sensory cortex of paraplegic humans. The motor activity paradigm consisted of tapping all four fingers against the palm of the hand in a squeezing motion. During the sensory scan, subjects' palms were rubbed by a wooden dowel. In the active movement task, normal subjects showed consistent blood oxygenation level dependent (BOLD) responses in both motor cortex and supplementary motor areas. SCI subjects showed more variability in their BOLD response strength and location, with a posterior shift in their maximal activity both in motor cortex and the SMA. These results corroborate the findings of Green et al. [6,8], indicating that SCI tends to a more posterior locus of cortical activity during movement. At the same time, the results of the sensory task showed that the sensory representation did not change in position. It is worth noting, however, that the sensory paradigm in this study was substantially different from those performed by the above mentioned research groups. The latter aimed their experiments on studying mainly propriostimulation in passive movement paradigms, while the experiment of Turner dealt only with exterostimulation. 


\subsection{Methods of Data Acquisition and Analysis}

\subsubsection{Subjects}

This study was approved by the Institutional Review Board (Memphis VAMC). Spinal cord injury patients and normal controls volunteered for the study and both gave informed (written) consent. There were no significant differences between the groups with respect to age or duration of injury. There were 15 control subjects, of which 3 were females. The SCI group included 24 patients, of which 2 were females.

\subsubsection{Data acquisition}

Movement-related cortical potentials were recorded in high-resolution EEG with repetitive movements of the middle or index finger. The motor potential component was selected for mapping and dipole source localization.

An electrode cap made of stretchable fabric and containing 121 tin electrodes encased in plastic holders was used in the recordings. There was an estimated average interelectrode distance of $2.25 \mathrm{~cm}$. Since activity of ocular muscles can introduce significant electrical interference, two channels were used to monitor horizontal and vertical eye movements. One channel was used for EMG recording. Individual scalp sites were slightly abraded through the hole in the top of each electrode and conducting gel was injected. Electrode impedances were lowered to below 5 kilo ohms. An electromagnetic digitizer (Polhemus, Colchester, VT) was 
used to sample the electrode positions on the scalp to establish the accurate location of electrode coordinates in three-dimensional space. The layout of the electrode cap is shown in Figure 2.4. The 128-channel DC amplifier system (Neuroscan, El Paso, TX) was calibrated. Data acquisition was set at a digitization rate of $500 \mathrm{~Hz}$ for continuous recording. Filter band pass was DC to 100 Hz. All EEG channels were recorded with respect to a linked ears reference. The Neuroscan system digitized all channels simultaneously. During off-line data analysis, three-second EEG intervals (epochs) were taken for averaging from the continuous recording. The epochs contained signals two seconds before and one second after the EMG onset. Each epoch of EEG recorded at 121 scalp electrodes was individually scrutinized for artifact and either included in the average or rejected. The averaged three-second waveforms from all 121 electrodes were then used to interpolate potentials in interelectrode spaces and map the motor potential distribution using a color-coded scale. This procedure was done using a software package for EEG research SCAN 4.1 (Neuroscan, El Paso, TX).

Individuals were seated on a comfortable chair or their wheelchair in a dimly lit room. In the voluntary movement tests, the subjects were asked to make self-paced, brisk finger movements at a rate of approximately one movement every 5-10 seconds, while their eyes were fixed on a distant point in the room. The EMG signal was recorded by bipolar surface electrodes placed over the appropriate muscles in the forearm (flexor digitorum sublimus). The EMG signal was used in off-line analysis to mark the time points of the EMG onset of each movement. In the passive movement tests, an operator moved the subject's finger at approximately the same rate as in the voluntary movement tests. A triggering device, specifically built for the passive movement tests, was used to trigger the acquisition computer. If time permitted, recordings were made of 


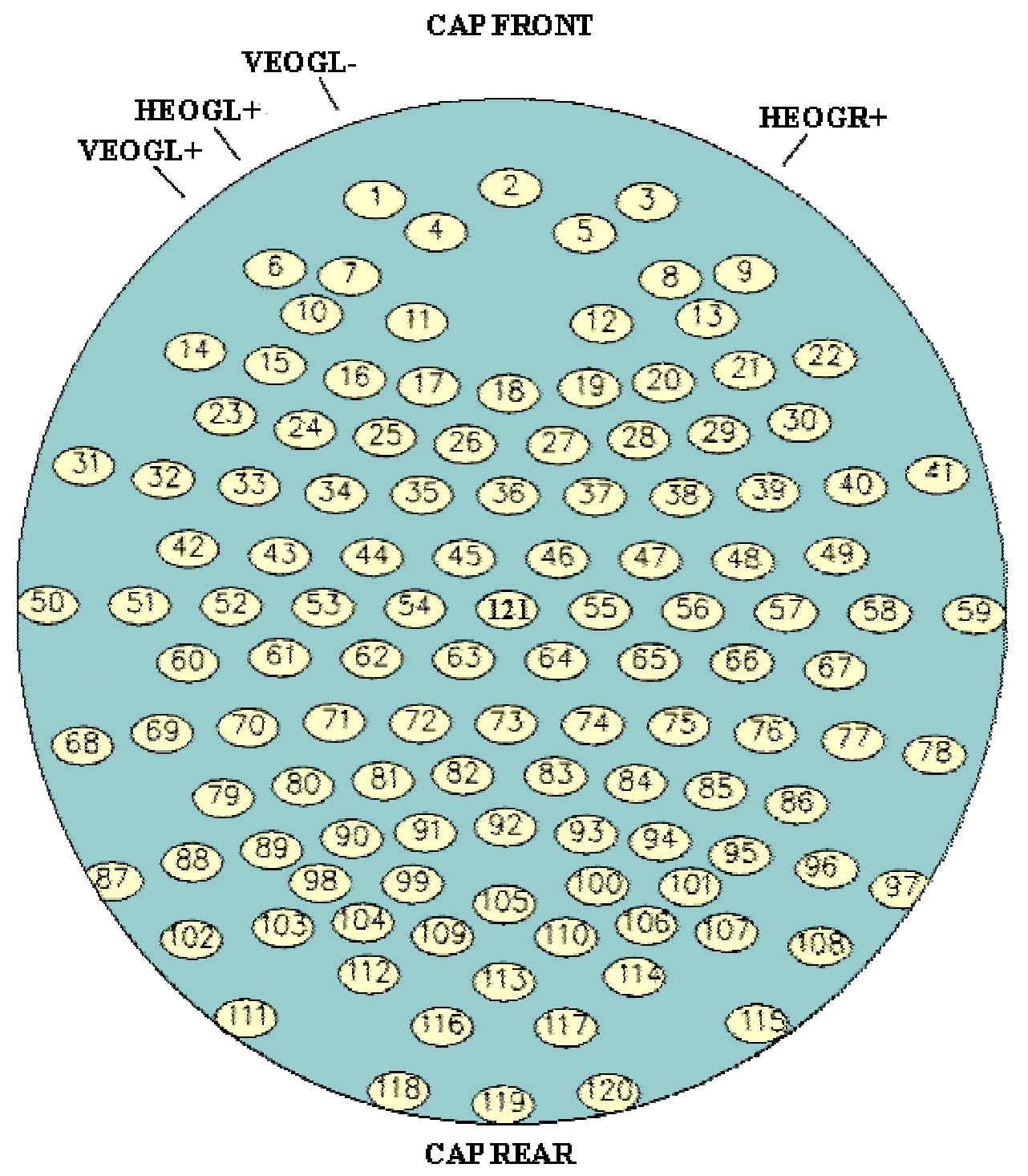

Figure 2.4. 121-electrode EEG cap layout.

self-paced and passive finger movements on each side sequentially. For each digit tested, the EEG was averaged off-line and saved in a separate file. 


\subsubsection{Triggering in passive movement tests}

Unlike in self-paced movement tests, there is no EMG activity in tests with passive movements. Even though occasional potential discharge can occur in the EMG channel, it cannot be used to determine the time instants of the beginning of each movement for the purpose of further processing. It has been proposed to use the operator's EMG activity as the trigger, similar to the setup in self-paced movement tests. However, the operator's movements involved in initiating the patient's finger movements are rather complex and activate a larger amount of muscles with various degrees of strength exerted, amplitude and rate of contraction. Different operators might also introduce additional factors of uncertainty. In order to maximally standardize the method of registering the time points of movements, yet keep it simple, it was decided to develop an electronic device which would trigger the data acquisition computer with a single pulse each time a finger movement was initiated. The acquisition program could be interfaced with external devices. The triggering is accomplished by mimicking the transistortransistor logic (TTL) signals expected by the data acquisition program. These are 8-bit, positive logic TTL pulses. The duration of pulses can range from 1 to about $10 \mathrm{~ms}$. Since we are interested in registering only one type of event, namely the beginning of finger movement, a onebit binary signal is enough to encode this amount of information.

In order to register a triggering mark in the acquisition system, it is necessary to send a TTL pulse to pin 8 on the 25-pin stimulus connector of the DC amplifier system. The triggering device and the acquisition system have to have common ground, which means that pin 25 of the stimulus connector must share the same potential with the ground of the triggering device. Figure 2.5 depicts the layout of these connections. All unused stimulus trigger pins, number 1 
through 7, must also be shorted to the ground to avoid possible spurious triggers. An electric diagram of the triggering device is shown in Figure 2.6. Figure 2.7 demonstrates a general view of the device as well as the way it is used in passive movement tests.

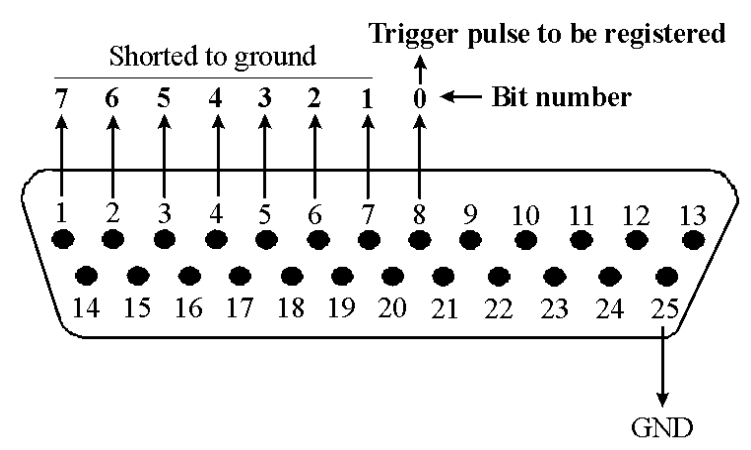

Figure 2.5. Back of the DC amplifier system stimulus connector.

\subsubsection{Analysis of physiological data}

The motor potential was averaged and graphed for all 121 electrode locations and displayed in electrical potential maps. A Euclidean metric was used to compare the location of the maxima of the scalp-distributed motor potentials in normal controls and SCI patients to determine whether there was a relationship between condition and motor potential location. The digitized three-dimensional electrode coordinates were projected onto a two-dimensional plane with the positive $\mathrm{x}$-axis pointing toward the right ear and the positive $y$-axis toward the nose. The two-dimensional coordinates were then used for subsequent analysis. The electrodes that contained the center of the peak of the motor potential were identified and the corresponding two-dimensional location of the geometrical center of the cluster formed by those electrodes was determined from the center of the head, the latter being at the intersection of the line connecting the two preauricular notches and the perpendicular thereto from the nasion notch. Using this technique, the center of activation due to various paradigms was compared on the subject's scalp. 


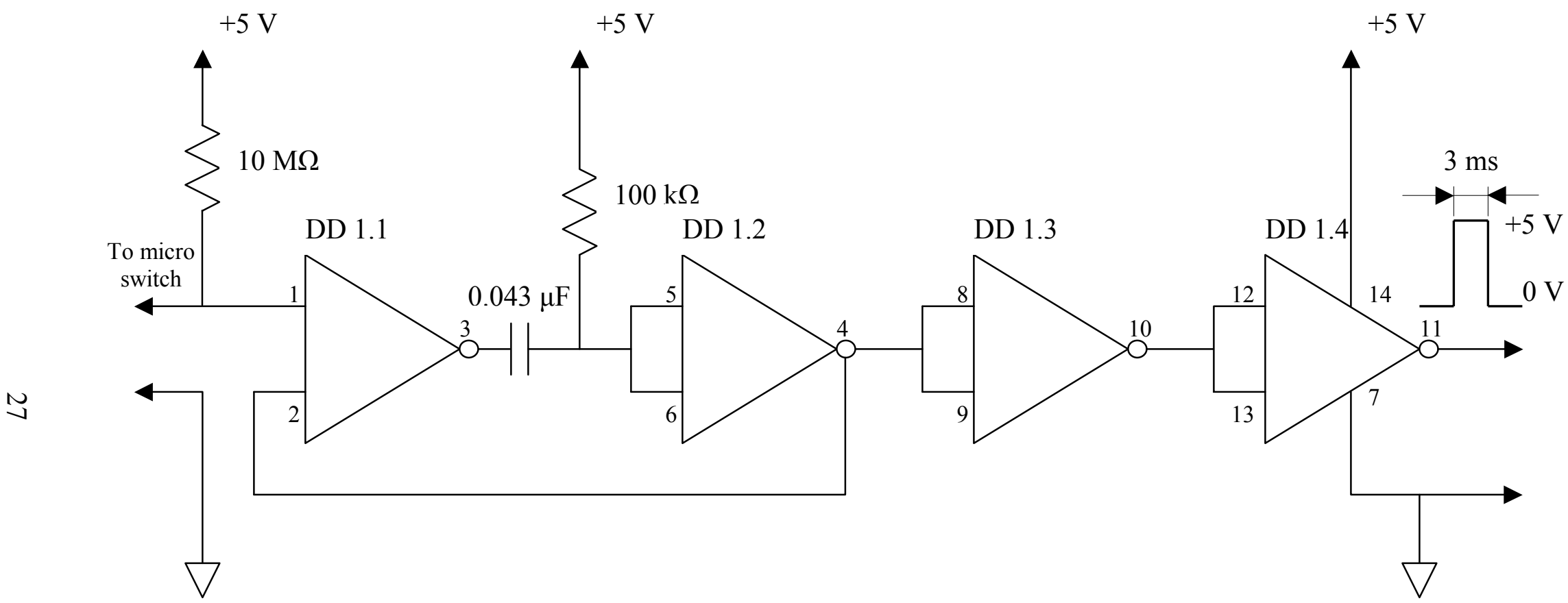

Figure 2.6. An electric diagram of the triggering device for passive movement tests. 


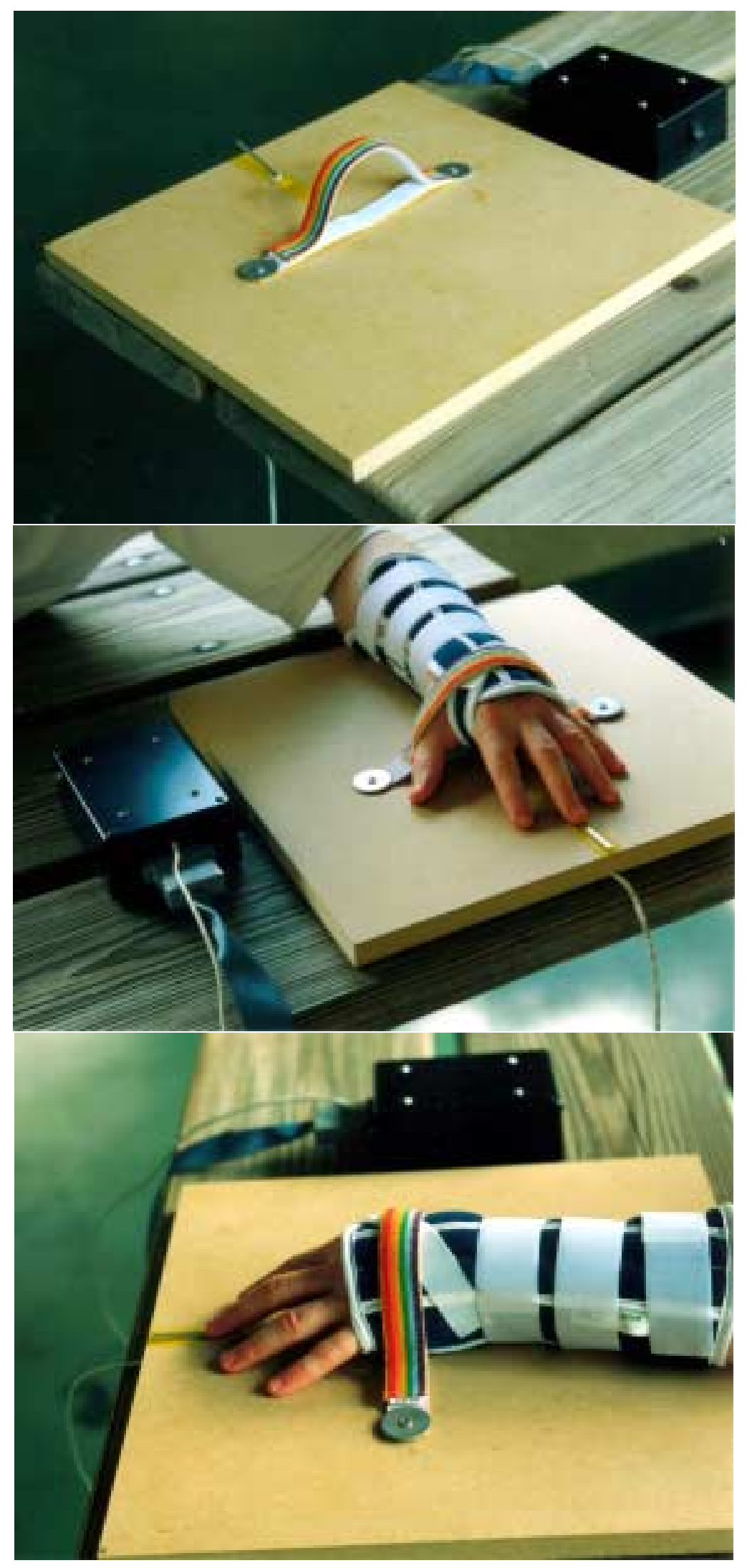

Figure 2.7. The triggering device for use in passive movement tests. 


\subsubsection{Dipole source analysis}

Dipole source analysis (DSA) is accomplished using a current reconstruction software package, CURRY Multi-modal neuroimaging [23]. This package uses several reconstruction algorithms, such as single dipole reconstruction, multiple dipole, and current density distribution. Individual specific MRI data are used to restrict the volume conductor geometry to the individual anatomy. The shape of the volume conductor model is determined by segmenting or separating the skin, skull, and brain surfaces from the MR image. These compartments are modeled using the boundary element method by assigning different appropriate conductivity values for each surface $[24,25]$. This allows accurate localization of cortical activity by restricting the model to neurophysiologically appropriate source locations such as the cortex. Calculations are based on a window of $50 \mathrm{~ms}$ before and after the motor potential peak for dipole source analysis. Dipole source analysis was limited to comparing the motor potential distribution fields with their putative sources in some patients and normal controls, for example contribution of the somatosensory area relative to the primary motor area.

\subsection{Biophysical Aspects of EEG and Source Reconstruction}

The various components of movement-related cortical potentials have been well described in the literature. It is also important to understand the nature of their generators. 
Determining the sources of movement-related cortical potentials provides the means for relating these electrophysiological events to the underlying anatomy of the brain.

In the brain and surrounding tissues, the material constants are approximately the following: conductivity, $\sigma \approx 10^{-1}(\Omega \cdot m)^{-1}$; dielectric constant, $\varepsilon \approx 10^{-9} \mathrm{~F} \cdot \mathrm{m}^{-1}$; and magnetic permeability, $\mu \approx 10^{-6} \mathrm{H} \cdot \mathrm{m}^{-1}$. No charge is accumulated at any time in the brain. It can be stated that for the current density $\vec{J}(\vec{x})$ or, for simplicity, $\vec{J}$, at any moment of time

$$
\nabla \bullet \vec{J}=0
$$

where $\vec{J}$ is the current density in $A \cdot m^{-2}$. Thus we may state that the current is stationary. In the case of a stationary current, the electric field $\vec{E}(\vec{x})$, or simply $\vec{E}$, is related to the electric potential $\Phi(\vec{x})$, or $\Phi$, by the following expression:

$$
\vec{E}=-\nabla \Phi
$$

In a conductive medium, Ohm's law is valid:

$$
\vec{J}=\vec{J}_{i}+\sigma \vec{E}
$$

where $\vec{J}_{i}$ is the current density injected in the medium, for example, a transmembrane current density caused by synaptic activity in a neuron.

Taking the divergence of both sides, we can write:

$$
\begin{aligned}
& \nabla \bullet \vec{J}=\nabla \bullet\left(\vec{J}_{i}+\sigma \vec{E}\right)=\nabla \bullet \vec{J}_{i}+\sigma \nabla \bullet \vec{E}=0 \\
& \nabla \bullet \vec{E}=-\frac{\nabla \bullet \vec{J}_{i}}{\sigma}
\end{aligned}
$$

Now we can write an expression that is called the Poisson equation for the potential field owing to an injected current: 


$$
\nabla^{2} \Phi=\frac{\nabla \bullet \vec{J}_{i}}{\sigma}
$$

It can be proved that the solution of the Poisson equation is the following [26]:

$$
\Phi(\vec{x})=-\frac{1}{4 \pi \sigma} \int_{V} \frac{\nabla \bullet \vec{J}_{i}}{\left|\vec{x}-\vec{x}^{\prime}\right|} d^{3} x^{\prime}
$$

which gives the values of the potential at a point $\vec{x}$ in the volume conductor from injected current densities $\vec{J}_{i}$ at points $\vec{x}^{\prime}$, lying at a distance $R$ from $\vec{x}, R=|\vec{x}-\vec{x}|$. The integral represents the summation over all current sources within the volume. The term $\left(-\nabla \bullet \vec{J}_{i}\right)$ plays the role of a divergence-type source density, and

$$
-\nabla \bullet \vec{J}_{i}=I_{V}
$$

has the dimensions of current per volume.

$$
\Phi(\vec{x})=\frac{1}{4 \pi \sigma} \int_{V} \frac{I_{V}}{\left|\vec{x}-\vec{x}^{\prime}\right|} d^{3} x^{\prime}
$$

It is convenient to represent the bioelectric sources as a volume source density function $I_{V \cdot}$. On this basis, the potential field is found as a solution to Poisson's equation:

$$
\nabla^{2} \Phi=-\frac{I_{V}}{\sigma}
$$

On the other hand, representation of the sources as an impressed current density $\vec{J}_{i}$ permits an identification with physical processes in the membrane. Let us consider the following vector identity.

$$
\nabla \bullet\left(\frac{\vec{J}_{i}}{R}\right)=\frac{1}{R} \nabla \bullet \vec{J}_{i}+\vec{J}_{i} \bullet \nabla\left(\frac{1}{R}\right)
$$

where $R=\left|\vec{x}-\vec{x}^{\prime}\right|$. 
If the integral of both sides is taken, which extends throughout the total volume in which sources are located, and the divergence theorem is used, then

$$
\int_{S} \frac{\vec{J}_{i} \bullet d S}{R}=0=\int_{V} \frac{\nabla \bullet \vec{J}_{i}}{R} d^{3} x^{\prime}+\int_{V} \vec{J}_{i} \bullet \nabla\left(\frac{1}{R}\right) d^{3} x^{\prime}
$$

The surface integral evaluates to zero since $\vec{J}_{i}=0$ over the bounding surface which lies beyond all sources. Consequently,

$$
\int_{V}-\frac{\nabla \bullet \vec{J}_{i}}{R} d^{3} x^{\prime}=\int_{V} \vec{J}_{i} \bullet \nabla\left(\frac{1}{R}\right) d^{3} x^{\prime}
$$

where $\nabla\left(\frac{1}{R}\right)=\nabla\left(\frac{1}{\left|\vec{x}-\vec{x}^{\prime}\right|}\right)=-\frac{\vec{x}-\vec{x}^{\prime}}{\left|\vec{x}-\vec{x}^{\prime}\right|^{3}}$.

Substituting (2.13) into (2.7) gives

$$
\Phi(\vec{x})=\frac{1}{4 \pi \sigma} \int_{V} \vec{J}_{i} d^{3} x^{\prime} \bullet \nabla\left(\frac{1}{R}\right)
$$

Now the potential field of a dipole source of strength $\vec{p}$ is

$$
\Phi(P)=\frac{1}{4 \pi \sigma} \vec{p} \bullet \nabla\left(\frac{1}{R}\right)
$$

A dipole is formed from a positive and a negative point source, each of equal magnitude $q$, separated by a vanishing small directed distance $\vec{l}$ such that $\vec{p}=\lim _{l \rightarrow 0} q \vec{l}$. The quantity $\vec{p}$ is the dipole moment. The field produced by the dipole can be evaluated by superposing the contribution from the component point sources. This can be accomplished by first considering both positive and negative point sources at the origin. The net field is zero since component fields are equal and opposite. Displacement of the positive source a distance $\vec{l}$, hence forming a 
dipole, results in a non-canceling component fields. The resultant is precisely the change in the field of the positive source due to displacing it.

Let us consider a point source $\delta$, where $\delta=0$ except at the origin, where it is infinite, but $\int_{V} \delta d V=1$. Designate by $\Phi_{0}$ the solution to the following partial differential equation:

$$
\nabla^{2} \Phi_{0}=\frac{\delta}{\sigma}
$$

Because of the spherical symmetry, $\Phi_{0}$ depends only on $r$ and writing the laplacian in spherical coordinates, we get

$$
\frac{1}{r^{2}} \frac{d}{d r}\left(r^{2} \frac{d \Phi_{0}}{d r}\right)=\frac{\delta}{\sigma}
$$

If we integrate with respect to $r$, then

$$
r^{2} \frac{d \Phi_{0}}{d r}=\frac{1}{\sigma} \int \delta r^{2} d r=\frac{1}{4 \pi \sigma} \int 4 \pi r^{2} \delta d r=\frac{1}{4 \pi \sigma} \int \delta d V=\frac{1}{4 \pi \sigma}
$$

A second integration yields

$$
\Phi_{0}=-\frac{1}{4 \pi \sigma R}
$$

In the notation used here, $\vec{x}$ is a position vector of the point at which $\Phi_{0}$ is evaluated, the delta source being at the origin.

Utilizing (2.19), we get

$$
\Phi=\frac{\partial}{\partial l}\left(\frac{q}{4 \pi \sigma R}\right)|\vec{l}|=\frac{q}{4 \pi \sigma} \nabla\left(\frac{1}{R}\right) \bullet \vec{l}
$$

By comparison of (2.15) with (2.14), $\vec{J}_{i} d V$ may be interpreted as an elemental dipole source.

Alternatively, $\vec{J}_{i}$ has the dimensions of a dipole moment per unit volume. Since isolated point sources with fields as given in (2.19) cannot exist in electrophysiological systems, the 
characterization of the bioelectric sources as a dipole distribution is natural. The electrostatic dual to $\vec{J}_{i}$ is, in the sense discussed here, the dielectric polarization, usually denoted $\vec{P}$.

We can obtain expressions for the potential field which imply certain fictitious or equivalent sources. The latter are normally expressed in terms of potential and/or current distributions over specialized or arbitrary surfaces. We will consider here a single cell immersed in a uniform conducting medium and obtain expressions for the potential field in terms of the potential distributions over the outer and inner membrane surfaces. The latter constitutes an effective, or equivalent, dipole-layer source.

Figure 2.8 illustrates a single cell lying in a volume conductor. The external membrane surface is $S_{0}$ and the membrane surface is $S_{i}$. We let $P(\vec{x})$ be an arbitrary field point in the external medium and $R=|\vec{x}-\vec{x}|$ be the distance from it to an arbitrary internal point.

We now apply Green's theorem

$$
\int_{V_{i}}\left(\Phi \nabla^{2} \psi-\psi \nabla^{2} \Phi\right) d V=\int_{S_{i}}\left(\Phi \frac{\partial \psi}{\partial n}-\psi \frac{\partial \Phi}{\partial n}\right) d a^{\prime \prime}
$$

and choose the scalar function $\psi=\frac{1}{R}$ and $\Phi$ equal to the potential field $\Phi_{i}$ in $V_{i}$. The field $\Phi_{i}$ arises from sources within the membrane under either resting or active conditions. Since $R$ cannot go to zero by virtue of $P$ being outside the region of integration, we have $\nabla^{2}\left(\frac{1}{R}\right)=0$.

Also since no sources exist within $V_{i}$ (the axoplasm is assumed to be a passive conductor), $\nabla^{2} \Phi_{i}=0$. Consequently,

$$
\int_{S_{i}} \Phi_{i} \frac{\partial\left(\frac{1}{R}\right)}{\partial n} d a^{\prime \prime}=\int_{S_{i}} \frac{1}{R} \frac{\partial \Phi_{i}}{\partial n} d a^{\prime \prime}
$$




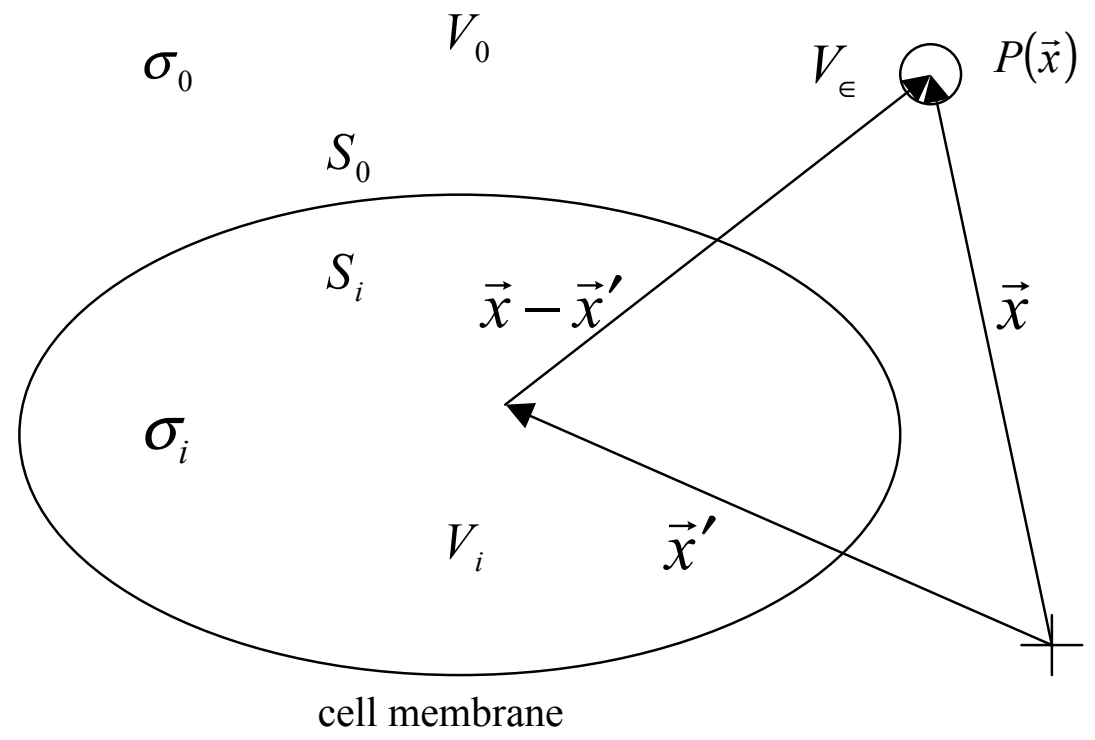

Figure 2.8. Single cell in a volume conductor of infinite extent.

We can replace

$$
-\left.\frac{\partial \Phi_{i}}{\partial n}\right|_{S_{i}}=\frac{J_{m}}{\sigma_{i}}
$$

where $\sigma_{i}$ is the conductivity in the axoplasm and $J_{m}$ is the outward membrane current density.

From (2.22) and (2.23), we have

$$
\int_{S_{i}} \Phi_{i} \frac{\partial\left(\frac{1}{R}\right)}{\partial n} d a^{\prime \prime}=-\frac{1}{\sigma_{i}} \int_{S_{i}}^{J_{m}} \frac{J^{\prime \prime}}{R} d a^{\prime \prime}
$$

If we multiply both sides by $\sigma_{i} / 4 \pi \sigma_{0}$, where $\sigma_{0}$ is the external conductivity, then we obtain

$$
\frac{1}{4 \pi \sigma_{0}} \int_{S_{i}}^{J_{m}} \frac{\sigma^{\prime}}{R} d a^{\prime \prime}=-\frac{1}{4 \pi} \frac{\sigma_{i}}{\sigma_{0}} \int_{S_{i}} \Phi_{i} \frac{\partial\left(\frac{1}{R}\right)}{\partial n} d a^{\prime \prime}
$$


Green's theorem is now applied to the external region. In this case we choose $\Phi=\Phi_{0}$, where $\Phi_{0}$ is the external potential field due to the bioelectric sources, while $\psi=\frac{1}{R}$ as before. Since there are no sources in the external region, $\nabla^{2} \Phi=0$. Noting that $\nabla^{2}\left(\frac{1}{R}\right)=-4 \pi \delta(R)$, the following result is obtained:

$$
\Phi_{0}(P(\vec{x}))=-\frac{1}{4 \pi} \int_{S_{0}} \frac{1}{R} \frac{\partial \Phi_{0}}{\partial n} d a^{\prime}+\frac{1}{4 \pi} \int_{S_{0}} \Phi_{0} \frac{\partial}{\partial n}\left(\frac{1}{R}\right) d a^{\prime}
$$

where $\Phi_{0}(P(\vec{x}))$ is the potential at the point $P(\vec{x})$, Figure 2.8. The sign given in this equation assumes the positive normal to be taken outward from $V_{i}$ and corresponds to the direction given in (2.22). The additional term, as compared with (2.22), is a consequence of the singularity of $\nabla^{2}\left(\frac{1}{R}\right)$ at $P$, which now lies in the region of integration. Substitution of $\nabla^{2}\left(\frac{1}{R}\right)=-4 \pi \delta(R)$ into (2.21) results in an expression in which the normal $\hat{n}$ is outward to $V_{0}$ (inward to $V_{i}$ ). To obtain (2.26) the sign of the left-hand side is changed while the direction of the positive normal on the right-hand side is reversed.

Since the membrane is very thin, we may assume negligible longitudinal current, in which case the transverse current must be continuous. That is,

$$
-\left.\frac{\partial \Phi_{0}}{\partial n}\right|_{S_{0}}=\frac{J_{m}}{\sigma_{0}}
$$

where $J_{m}$ is the same as that in (2.23) and $\sigma_{0}$ is the external conductivity. Using (2.27) and adding (2.25) and (2.26) yields the following expression for the potential $\Phi_{0}(P)$ :

$$
\Phi_{0}=\frac{1}{4 \pi \sigma_{0}}\left(\int_{S_{0}} \frac{J_{m}}{R} d a^{\prime}-\int_{S_{i}} \frac{J_{m}}{R} d a^{\prime \prime}\right)-\frac{1}{4 \pi}\left(\frac{\sigma_{i}}{\sigma_{0}} \int_{S_{i}} \Phi_{i} \frac{\partial}{\partial n}\left(\frac{1}{R}\right) d a^{\prime \prime}-\int_{S_{0}} \Phi_{0} \frac{\partial}{\partial n}\left(\frac{1}{R}\right) d a^{\prime}\right)
$$


We will consider field points whose distance to the membrane is large compared with the membrane thickness $m_{d}$. Since $m_{d}$ is actually extremely small, this is not a significant restriction. Under this condition, the integrals in the first group can be combined into a doublelayer representation using a Taylor series expansion in three dimensions. Also, the integrals in the second group may both be integrated over a mean surface $S$ which lies between $S_{0}$ and $S_{i}$. (2.28) then takes on the following form (where the positive surface normal is outward from the cell, i.e., from $S)$ :

$$
\begin{aligned}
& \frac{1}{\left|\vec{x}-\vec{x}^{\prime}+\hat{n} m_{d}\right|} \approx \frac{1}{\left|\vec{x}-\vec{x}^{\prime}\right|}+\hat{n} m_{d} \bullet \nabla^{\prime}\left(\frac{1}{\left|\vec{x}-\vec{x}^{\prime}\right|}\right)=\frac{1}{R}+\hat{n} m_{d} \bullet \nabla^{\prime}\left(\frac{1}{R}\right) \\
& \Phi_{0}(P)=\frac{1}{4 \pi \sigma_{0}}\left(\int_{S_{0}} \frac{J_{m}}{\left|\vec{x}-\vec{x}^{\prime}\right|} d a^{\prime}-\int_{S_{i}} \frac{J_{m}}{\left|\vec{x}-\vec{x}^{\prime}+\hat{n} m_{d}\right|} d a^{\prime \prime}\right)-\frac{1}{4 \pi} \int_{S}\left(\frac{\sigma_{i}}{\sigma_{0}} \Phi_{i}-\Phi_{0}\right) \nabla^{\prime}\left(\frac{1}{R}\right) \cdot d \vec{S} \\
& =\frac{1}{4 \pi \sigma_{0}}\left(\int_{S_{0}} \frac{J_{m} d a^{\prime}}{\left|\vec{x}-\vec{x}^{\prime}\right|}-\int_{S_{i}} \frac{J_{m} d a^{\prime \prime}}{\left|\vec{x}-\vec{x}^{\prime}\right|}-\int_{S_{i}} J_{m} \hat{n} m_{d} \bullet \nabla^{\prime}\left(\frac{1}{R}\right) d a^{\prime \prime}\right)-\frac{1}{4 \pi} \int_{S}\left(\frac{\sigma_{i}}{\sigma_{0}} \Phi_{i}-\Phi_{0}\right) \nabla^{\prime}\left(\frac{1}{R}\right) \bullet d \vec{S} \\
& \approx \frac{1}{4 \pi \sigma_{0}}\left(\int_{S} m_{d} J_{m} \nabla^{\prime}\left(\frac{1}{R}\right) \bullet d \vec{S}\right)-\frac{1}{4 \pi} \int_{S}\left(\frac{\sigma_{i}}{\sigma_{0}} \Phi_{i}-\Phi_{0}\right) \nabla^{\prime}\left(\frac{1}{R}\right) \bullet d \vec{S}
\end{aligned}
$$

The quantity

$$
-\nabla^{\prime}\left(\frac{1}{R}\right) \cdot d \vec{S}=\frac{\hat{\alpha}_{r} \bullet d \vec{S}}{R^{2}}=d \Omega
$$

where $\hat{\alpha}_{r}$ is a unit vector from the field to source point, corresponds to the solid angle subtended at $\mathrm{P}$ by a surface element $d S$. If we designate this quantity by $d \Omega$, then $(2.30)$ can be expressed as

$$
\Phi_{0}(P)=\frac{1}{4 \pi} \int_{S}\left(\frac{\sigma_{i}}{\sigma_{0}} \Phi_{i}-\Phi_{0}-\frac{m_{d} J_{m}}{\sigma_{0}}\right) d \Omega
$$

Equation (2.32) can be simplified since the third term is ordinarily negligible. 


$$
\Phi(P)=\frac{1}{4 \pi} \int_{S}\left(\frac{\sigma_{i}}{\sigma_{0}} \Phi_{i}-\Phi_{0}\right) d \Omega
$$

When $\sigma_{i}=\sigma_{0}$, then denoting $\left(\Phi_{i}-\Phi_{0}\right)=V$, where $V$ is the transmembrane potential, we get

$$
\Phi(P)=\frac{1}{4 \pi} \int_{S} V d \Omega \quad\left(\sigma_{i}=\sigma_{0}\right)
$$

A further approximation is based on $\Phi_{0}$ being relatively constant so that its contribution is small. In this case we can take it to be a zero reference and get

$$
\Phi(P)=\frac{1}{4 \pi} \frac{\sigma_{i}}{\sigma_{0}} \int_{S} V d \Omega
$$

For an extensive medium this approximation is well justified. The coefficient in (2.35) can be thought of as expressing a division of the applied voltage between the internal and external loads.

The general expression of (2.33) can be rewritten based on the definition of the solid angle given in (2.31)

$$
\Phi(P)=\frac{1}{4 \pi \sigma_{0}} \int_{S}\left(\sigma_{0} \Phi_{0}-\sigma_{i} \Phi_{i}\right) d \vec{S} \bullet \nabla^{\prime}\left(\frac{1}{R}\right)
$$

Comparison with (2.15) shows that $\left(\sigma_{0} \Phi_{0}-\sigma_{i} \Phi_{i}\right) d \vec{S}$ behaves like a dipole of magnitude $\left(\sigma_{0} \Phi_{0}-\sigma_{i} \Phi_{i}\right) d S$ having the direction of the outward surface normal. The quantity $\left(\sigma_{0} \Phi_{0}-\sigma_{i} \Phi_{i}\right)$ can be interpreted, therefore, as a dipole moment per unit area or double layer in a $\sigma_{0}$ medium. The result fits the general description given in (2.14) except that the source is no longer directly identified with the underlying phenomena responsible for converting energy into the electrical form. Consequently, the source is an equivalent one and is capable of giving the correct values everywhere except in the membrane or, because of the approximations, very close to the membrane. 
From a field theoretic standpoint the membrane is treated as an infinitely thin surface across which the potential function $(\sigma \Phi)$ is discontinuous but whose normal derivative is continuous. Such a boundary condition is characteristic of a double-layer source located at the surface. Moreover, the strength of the dipole layer must equal the discontinuity in $(\sigma \Phi)$, and this is precisely the condition imposed by (2.36).

For a medium containing many small active cells, (2.36) applies to each such cell. However, each differential cell can be approximated by a single dipole which is the integral of

$$
\left(\sigma_{0} \Phi_{0}-\sigma_{i} \Phi_{i}\right) \hat{n} d S
$$

over its surface. If the density is high enough, this permits specifying a dipole moment per unit volume $\vec{J}_{i}$. The potential field may now be evaluated by (2.14). Alternatively, the divergencetype source

$$
-\nabla \cdot \vec{J}_{i}=I_{V}
$$

may be used in (2.9). $\vec{J}_{i}$ is an effective dipole moment per unit volume, and the representation here is an equivalent one. The accuracy of the computed field outside the source region depends only on the accuracy with which $\vec{J}_{i}$ represents the source density.

Another expression for the potential caused by one dipole:

$$
\Phi(P)=\frac{p\left(\vec{x}^{\prime}\right)}{4 \pi \sigma} \nabla^{\prime}\left(\frac{1}{R}\right) \cdot d \vec{S}
$$

or

$$
\Phi(P)=\frac{p\left(\vec{x}^{\prime}\right) \cos \theta}{4 \pi \sigma R^{2}} d S
$$


From (2.40) it can be concluded that in the case of a single dipole at the membrane, $\Phi$ decreases with the square of the distance $R$ to the source and it is also proportional to $\cos \theta$, thus it is maximal when $\vec{R}$ is perpendicular to the surface $d S$.

In many cases, the membrane depolarization (or hyperpolarization) does not remain limited to a small piece of membrane; rather, it spreads over a more or less extended membrane surface. In this way the equivalent current source cannot be accounted for in terms of a single dipole. It is necessary to assume that there exists a dipole layer at the membrane.

For a dipole layer located at the surface of the membrane, the effects of all individual dipoles have to be summed. Therefore, (2.40) becomes:

$$
\Phi(P)=\frac{1}{4 \pi \sigma} \int_{S} p\left(\vec{x}^{\prime}\right) \nabla^{\prime}\left(\frac{1}{R}\right) \bullet d \vec{S}
$$

or

$$
\Phi(P)=\frac{1}{4 \pi \sigma} \int_{S} p\left(\vec{x}^{\prime}\right) d \Omega
$$

From (2.42), it can be noted that, if $p$ is constant over the closed membrane surface $S$, then $\Phi(P)=0$, because the integral of the solid angle $d \Omega$ over an external closed surface is zero. Thus, in order to obtain a value of $\Phi(P)$ different from zero, $p$ must vary over the membrane surface; in other words, the membrane potential must vary over the surface owing, for example, to local synaptic activity.

The mathematical derivations presented here give an answer to the forward problem. They show calculation of the potential distribution given the underlying current source is known. The source reconstruction package [23] employed in this project uses an iterative procedure to derive current sources based on formula (2.39). 


\subsection{Calculation of the Coordinates of the Sites of Activation}

The MP distribution is used as the primary determinant of reorganization. It has been alleged that the MP has the same physiologic function in normal controls and patients. In both groups, there is a surface negativity accompanying movements of the fingers that is contralateral to the BP. The motor potential of the MRCP is selected for statistical analysis because it is generated by the contralateral motor area, simplifying its localization.

The three dimensional coordinates of all EEG electrodes, as well as those of the nasion, left and right preauricular fiduciary points, are obtained using an electromagnetic digitizer. Figure 2.9 shows a head surface rendered from a set of MRI images of one of the project participants. The digitized three-dimensional coordinates are used to locate the EEG electrodes on the rendered head model with respect to the three landmark points. Comparisons between groups are accomplished by normalizing the head size using the distance between the left and right preauricular landmark points. The coordinates of each electrode on the $x-y$ plane, therefore, become numbers from -1 to 1 . The location of the active area on the scalp in any motor task is determined by the location of the electrode that shows EEG signal with the largest amplitude of the motor potential. However, since the surface density of electrodes on the scalp is rather high, there might be two or in some cases even three electrodes that cover the area on the scalp where most of the activity takes place. The amplitudes of the motor potential in such electrodes may differ insignificantly. Therefore, it has been decided to identify from one to three electrodes in which the amplitude of the motor potential is the largest and varies within $5 \%$ of the maximum value as the most representative ones of the spatial location of the active area. 

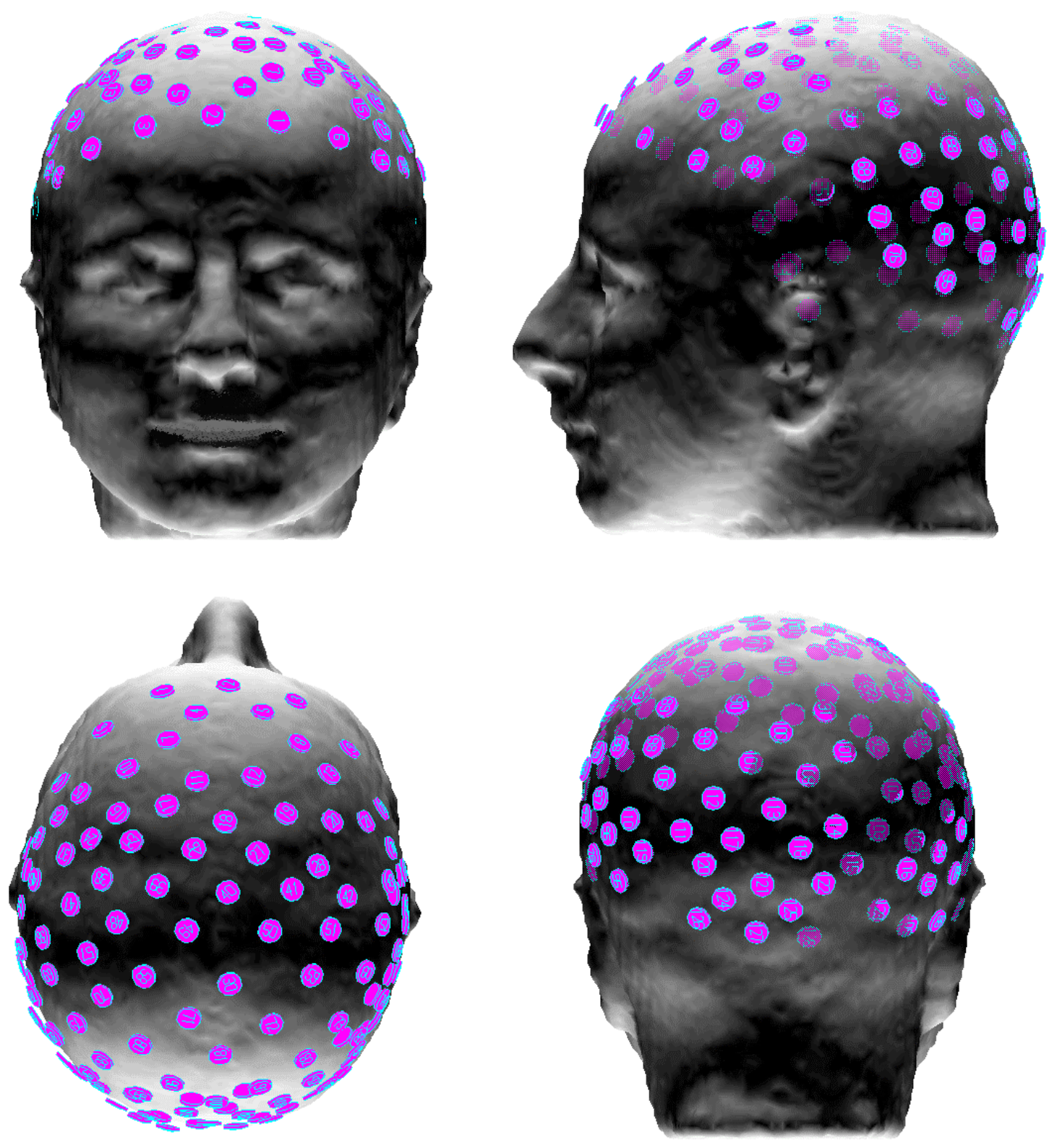

Figure 2.9. A head model rendered from MRI images with EEG electrodes.

The two-dimensional coordinates of the center of such a cluster of motor activity are then calculated from the corresponding coordinates of the electrodes. If one electrode covers the site of activity, then it simply represents the location of interest and its coordinates are normalized. If 
two electrodes are determined to be in the active area, then the center of the line connecting the two electrodes is calculated. If three electrodes are within the 5\% potential range, then the center of gravity of the triangle formed by these electrodes is calculated being on the intersection of any two medians. These electrodes are assumed to contribute equally to the localization of the active area and therefore treated as having equal weight. A Java program has been written to facilitate the numerous calculations. The program prompts the user to enter the coordinates of the landmark points, the number of electrodes in the active area, and the coordinates thereof. It outputs the normalized coordinates of the center of the cluster formed by these electrodes as described. The source code of the program Coordinates.java is provided in the Appendix.

\subsection{Statistical Analysis: Two-Sample $t$-Test for Independent Samples}

In a two-sample hypothesis-testing problem, the underlying parameters of two different populations, neither of whose values is assumed known, are compared. Two different experimental designs can be used to assess this relationship: a longitudinal or follow-up study and a cross-sectional study. There is an important difference between these two designs. The first study represents a paired-sample design. The second study represents an independent-sample design. Two samples are said to be paired when each data point of the first sample is matched and is related to a unique data point of the second sample. Two samples are said to be independent when the data points in one sample are unrelated to the data points in the second sample. Since two independent subject groups, controls and SCI patients, are used in this project, this is a cross-sectional study. 


\subsubsection{Equal variances case}

Let us assume that the random variable is normally distributed in the first group with mean $\mu_{1}$ and variance $\sigma_{1}^{2}$ and in the second group with mean $\mu_{2}$ and variance $\sigma_{2}^{2}$. We want to test the hypothesis $\mathrm{H}_{0}: \mu_{1}=\mu_{2}$ versus $\mathrm{H}_{1}: \mu_{1} \neq \mu_{2}$. Assume that the underlying variances in the two groups are the same, i.e. $\sigma_{1}{ }^{2}=\sigma_{2}{ }^{2}=\sigma^{2}$. The means and variances in the two samples are denoted by $\bar{x}_{1}, \bar{x}_{2}, \mathrm{~s}_{1}^{2}, \mathrm{~s}_{2}^{2}$ respectively.

The significance test is based on the difference between the two sample means, $\bar{x}_{1}-\bar{x}_{2}$. If this difference is far from 0 , then $\mathrm{H}_{0}$ will be rejected; otherwise, it will be accepted. Thus, we study the behavior of $\bar{x}_{1}-\bar{x}_{2}$ under $\mathrm{H}_{0}$. We know that $\bar{x}_{1}$ is normally distributed with mean $\mu_{1}$ and variance $\sigma^{2} / \mathrm{n}_{1}$ and that $\bar{x}_{2}$ is normally distributed with mean $\mu_{2}$ and variance $\sigma^{2} / \mathrm{n}_{2}$. Hence, since the two samples are independent, $\bar{x}_{1}-\bar{x}_{2}$ is normally distributed with mean $\mu_{1}-\mu_{2}$ and variance $\sigma^{2}\left(1 / \mathrm{n}_{1}+1 / \mathrm{n}_{2}\right)$. In symbols,

$$
\bar{x}_{1}-\bar{x}_{2} \sim N\left[\mu_{1}-\mu_{2}, \sigma^{2}\left(\frac{1}{n_{1}}+\frac{1}{n_{2}}\right)\right]
$$

Under $\mathrm{H}_{0}$, we know that $\mu_{1}=\mu_{2}$. Thus, the previous equation reduces to

$$
\bar{x}_{1}-\bar{x}_{2} \sim N\left[0, \sigma^{2}\left(\frac{1}{n_{1}}+\frac{1}{n_{2}}\right)\right]
$$

If $\sigma^{2}$ were known, then $\bar{x}_{1}-\bar{x}_{2}$ could be divided by $\sigma \sqrt{\frac{1}{n_{1}}+\frac{1}{n_{2}}}$. Then the test statistic

$$
\frac{\bar{x}_{1}-\bar{x}_{2}}{\sigma \sqrt{\frac{1}{n_{1}}+\frac{1}{n_{2}}}} \sim \mathrm{N}(0,1)
$$


can be used as a basis for the hypothesis test. However, $\sigma^{2}$ in general is unknown and must be estimated from the data. From the first and second sample, the sample variances are $\mathrm{s}_{1}{ }^{2}$ and $\mathrm{s}_{2}{ }^{2}$, respectively. The best estimate of the population variance $\sigma^{2}$, which is denoted by $s^{2}$, is given by a weighted average of the two sample variances, where the weights are the number of degrees of freedom in each sample. The pooled estimate of the variance from two independent samples is given by

$$
s^{2}=\frac{\left(n_{1}-1\right) s_{1}^{2}+\left(n_{2}-1\right) s_{2}^{2}}{n_{1}+n_{2}-2}
$$

In particular, $\mathrm{s}^{2}$ will then have $\mathrm{n}_{1}-1$ degrees of freedom from the first sample and $\mathrm{n}_{2}-1$ degrees of freedom from the second sample, or $n_{1}+n_{2}-2$ overall. $s$ can then be substituted for $\sigma$ into the test statistic and the resulting test statistic can then be shown to follow a $t$ distribution with $\mathrm{n}_{1}+\mathrm{n}_{2}-2$ degrees of freedom rather than an $\mathrm{N}(0,1)$ distribution, since $\sigma^{2}$ is unknown.

Therefore, we compute the test statistic:

$$
t=\frac{\bar{x}_{1}-\bar{x}_{2}}{s \sqrt{\frac{1}{n_{1}}+\frac{1}{n_{2}}}}
$$

If $t>t_{n_{1}+n_{2}-2,1-\alpha / 2}$ or $t<-t_{n_{1}+n_{2}-2,1-\alpha / 2}$ then $\mathrm{H}_{0}$ is rejected. Otherwise, $\mathrm{H}_{0}$ is accepted.

Similarly, a $p$-value can be computed for the test. The computation of the $p$-value will depend on whether $\bar{x}_{1} \leq \bar{x}_{2}(t \leq 0)$ or $\bar{x}_{1}>\bar{x}_{2}(t>0)$. In each case, the $p$-value corresponds to the probability of obtaining a test statistic at least as extreme as the observed value $t$. 


\subsubsection{Unequal variances case}

In the preceding discussion, it was assumed that the underlying variances of the two samples were the same. The common variance was then estimated using a weighted average of the individual sample variances. In this section, a significance test to validate this assumption is developed. In particular, we want to test the hypothesis $\mathrm{H}_{0}: \sigma_{1}{ }^{2}=\sigma_{2}{ }^{2}$ versus $\mathrm{H}_{1}: \sigma_{1}{ }^{2} \neq \sigma_{2}{ }^{2}$, where the two samples are assumed to be independent random samples from an $\mathrm{N}\left(\mu_{1}, \sigma_{1}^{2}\right)$ and $\mathrm{N}\left(\mu_{2}, \sigma_{2}{ }^{2}\right)$ distribution, respectively. The significance test should be based on the ratio of the sample variances $\left(\mathrm{s}_{1}{ }^{2} / \mathrm{s}_{2}{ }^{2}\right)$. Thus, $\mathrm{H}_{0}$ would be rejected if the variance ratio is either too large or too small and accepted otherwise. To implement this test, the sampling distribution of $\mathrm{s}_{1}{ }^{2} / \mathrm{s}_{2}{ }^{2}$ under the null hypothesis is to be found.

\subsubsection{The F distribution}

The distribution of the variance ratio $\left(\mathrm{s}_{1}{ }^{2} / \mathrm{s}_{2}{ }^{2}\right)$ was studied by the statisticians Fisher and Snedecor. It can be shown that the variance ratio follows an $F$ distribution under the null hypothesis that $\sigma_{1}{ }^{2}=\sigma_{2}{ }^{2}$. There is no unique $F$ distribution but instead a family of $F$ distributions. This family is indexed by two parameters termed the numerator and denominator degrees of freedom (df), respectively. Specifically, if the sample sizes of the first and second samples are $\mathrm{n}_{1}$ and $\mathrm{n}_{2}$, then the variance ratio follows an $F$ distribution with $\mathrm{n}_{1}-1$ (numerator $\mathrm{df}$ ) and $\mathrm{n}_{2}-1$ (denominator df), which is denoted by $F_{n_{1}-1, n_{2}-1}$. The $F$ distribution is generally positively skewed, with the skewness dependent on the relative magnitudes of the two degrees of freedom. 


\subsubsection{The F test}

We now return to the significance test for the equality of two variances. We test the hypothesis $H_{0}: \sigma_{1}^{2}=\sigma_{2}^{2}$ versus $H_{1}: \sigma_{1}^{2} \neq \sigma_{2}^{2}$ with significance level $\alpha$. The test will be based on the variance ratio $\mathrm{s}_{1}{ }^{2} / \mathrm{s}_{2}{ }^{2}$, which under $\mathrm{H}_{0}$ follows an $F$ distribution with $\mathrm{n}_{1}-1$ and $\mathrm{n}_{2}-1$ degrees of freedom. Since this is a two-sided test, we will reject $\mathrm{H}_{0}$ for both small and large values of $\mathrm{s}_{1}{ }^{2} / \mathrm{s}_{2}{ }^{2}$. This procedure can be made more specific as follows. We compute the test statistic $F=$ $\mathrm{s}_{1}{ }^{2} / \mathrm{s}_{2}{ }^{2}$. If $F>F_{n_{1}-1, n_{2}-1,1-\alpha / 2}$ or $F<F_{n_{1}-1, n_{2}-1, \alpha / 2}$ then $\mathrm{H}_{0}$ is rejected. If $F_{n_{1}-1, n_{2}-1, \alpha / 2} \leq F \leq F_{n_{1}-1, n_{2}-1,1-\alpha / 2}$ then $\mathrm{H}_{0}$ is accepted.

\subsubsection{Two-sample t-test for independent samples with unequal variances}

The $F$ test for the equality of two variances from two independent, normally distributed samples was presented in the preceding discussion. If the two variances are significantly different, then a two-sample $t$-test for independent samples with unequal variances, which is presented here, is to be used.

Specifically, assume that there are two normally distributed samples, where the first sample is a random sample of size $\mathrm{n}_{1}$ from an $\mathrm{N}\left(\mu_{1}, \sigma_{1}{ }^{2}\right)$ distribution, the second sample is a random sample from an $\mathrm{N}\left(\mu_{2}, \sigma_{2}{ }^{2}\right)$ distribution, and $\sigma_{1}{ }^{2} \neq \sigma_{2}{ }^{2}$. We again wish to test the hypothesis $\mathrm{H}_{0}: \mu_{1}=\mu_{2}$ versus $\mathrm{H}_{1}: \mu_{1} \neq \mu_{2}$. This problem is referred to as the Behrens-Fisher problem.

It still makes sense to base the significance test on the difference between the sample means $\bar{x}_{1}-\bar{x}_{2}$. Under either hypothesis, $\bar{x}_{1}$ is normally distributed with mean $\mu_{1}$ and variance $\sigma_{1}{ }^{2} / \mathrm{n}_{1}$, and $\bar{x}_{2}$ is normally distributed with mean $\mu_{2}$ and variance $\sigma_{2}{ }^{2} / \mathrm{n}_{2}$. Hence it follows that 


$$
\bar{x}_{1}-\bar{x}_{2} \sim N\left(\mu_{1}-\mu_{2}, \frac{\sigma_{1}^{2}}{n_{1}}+\frac{\sigma_{2}^{2}}{n_{2}}\right)
$$

Under $\mathrm{H}_{0}, \mu_{1}-\mu_{2}=0$. Therefore, we can obtain

$$
\bar{x}_{1}-\bar{x}_{2} \sim N\left(0, \frac{\sigma_{1}^{2}}{n_{1}}+\frac{\sigma_{2}^{2}}{n_{2}}\right)
$$

If $\sigma_{1}^{2}$ and $\sigma_{2}^{2}$ were known, then the test statistic

$$
\frac{\bar{x}_{1}-\bar{x}_{2}}{\sqrt{\frac{\sigma_{1}^{2}}{n_{1}}+\frac{\sigma_{2}^{2}}{n_{2}}}}
$$

could be used for the significance test, which under $\mathrm{H}_{0}$ would be distributed as an $\mathrm{N}(0,1)$ distribution. However, $\sigma_{1}{ }^{2}$ and $\sigma_{2}{ }^{2}$ are usually unknown and are estimated by $\mathbf{s}_{1}{ }^{2}$ and $\mathbf{s}_{2}{ }^{2}$ respectively, i.e. the sample variances in the two samples. If $\mathrm{s}_{1}{ }^{2}$ is substituted for $\sigma_{1}{ }^{2}$ and $\mathrm{s}_{2}{ }^{2}$ for $\sigma_{2}^{2}$ in the last equation, then the following test statistic is obtained:

$$
t=\frac{\bar{x}_{1}-\bar{x}_{2}}{\sqrt{\frac{s_{1}^{2}}{n_{1}}+\frac{s_{2}^{2}}{n_{2}}}}
$$

The exact distribution of $t$ under $\mathrm{H}_{0}$ is difficult to derive. However, several approximate solutions have been proposed that have appropriate type I error. The Satterthwaite approximation is used here. Its advantage is its easy implementation using the ordinary $t$ tables.

First, we compute the test statistic $t$ and the approximate degrees of freedom $d^{\prime}$, where

$$
d^{\prime}=\frac{\left(\frac{s_{1}^{2}}{n_{1}}+\frac{s_{2}^{2}}{n_{2}}\right)^{2}}{\frac{\left(\frac{s_{1}^{2}}{n_{1}}\right)^{2}}{n_{1}-1}+\frac{\left(\frac{s_{2}^{2}}{n_{2}}\right)^{2}}{n_{2}-1}}
$$


Then we round $d^{\prime}$ down to the nearest integer $d^{\prime \prime}$. If $t>t_{d^{\prime \prime}, 1-\alpha / 2}$ or $t<-t_{d^{\prime \prime}, 1-\alpha / 2}$ then $\mathrm{H}_{0}$ is rejected. If $-t_{d^{\prime \prime}, 1-\alpha / 2} \leq t \leq-t_{d^{\prime \prime}, 1-\alpha / 2}$ then $\mathrm{H}_{0}$ is accepted.

Two procedures for comparing two means from independent, normally distributed samples have been presented. The first step in this process is to test for the equality of the two variances using the $F$ test. If this test is not significant, then the $t$-test with equal variances is used, otherwise, the $t$-test with unequal variances is used. This overall strategy is illustrated in Figure 2.10.

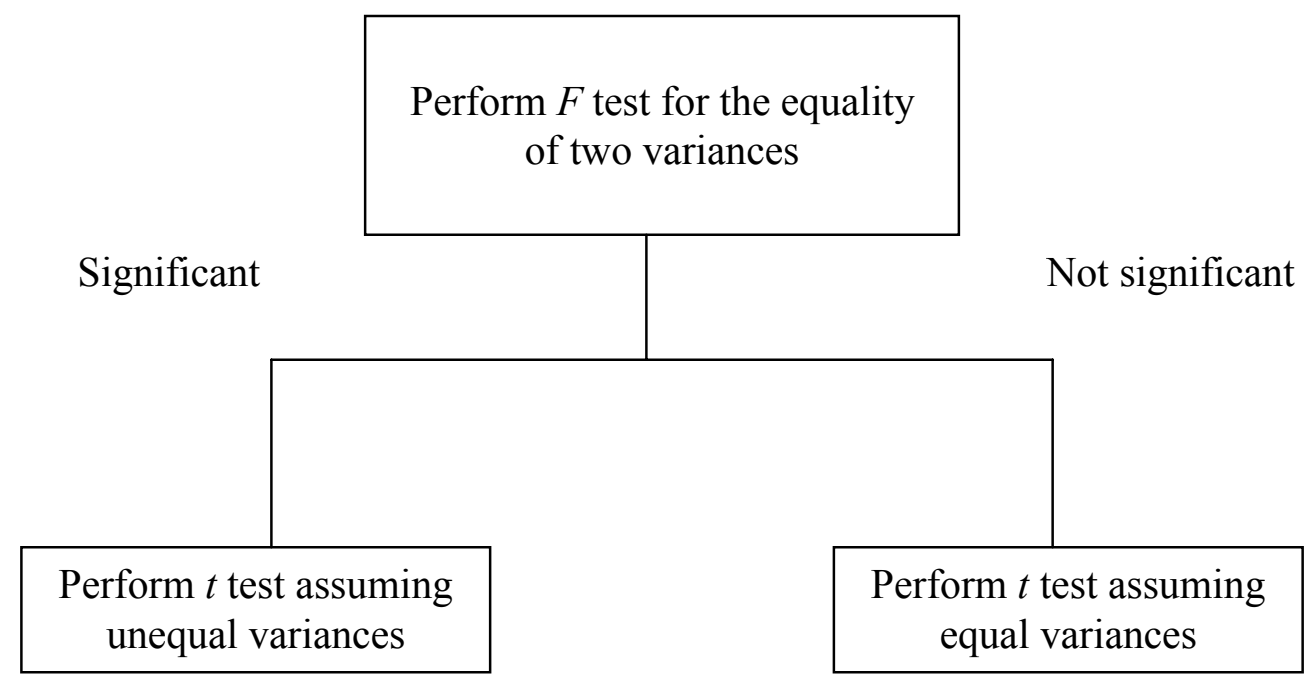

Figure 2.10. Strategy for testing for the equality of means in two independent, normally distributed samples. 


\section{CHAPTER 3. RESULTS AND CONCLUSIONS}

\subsection{Introduction}

In this chapter, the results from EEG motor potential mappings, source reconstructions and statistical analysis of the data are displayed. First, two-dimensional coordinates of the sites of activation are calculated for each subject as described in the previous chapter. Analogous calculations are then performed using the method of source reconstruction which shows the location of putative sources in the brain responsible for the generation of the potential patterns. The source reconstruction technique allows localization of active areas using individual anatomy of the brain available from MRI images. Statistical analysis of the location of sources is performed for the two major groups, SCI patients and control subjects. The results and conclusions are discussed at the end of this chapter.

\subsection{Statistical Comparison of the Sites of Cortical Activation}

In this study, we use an independent-sample design for statistical analysis, since two completely different groups of subjects are being compared. This is a cross-sectional study.

Figures 3.1 and 3.2 show an averaged three-second epoch recorded from the site of activation in a paraplegic patient and a normal control subject, respectively. Each of these 


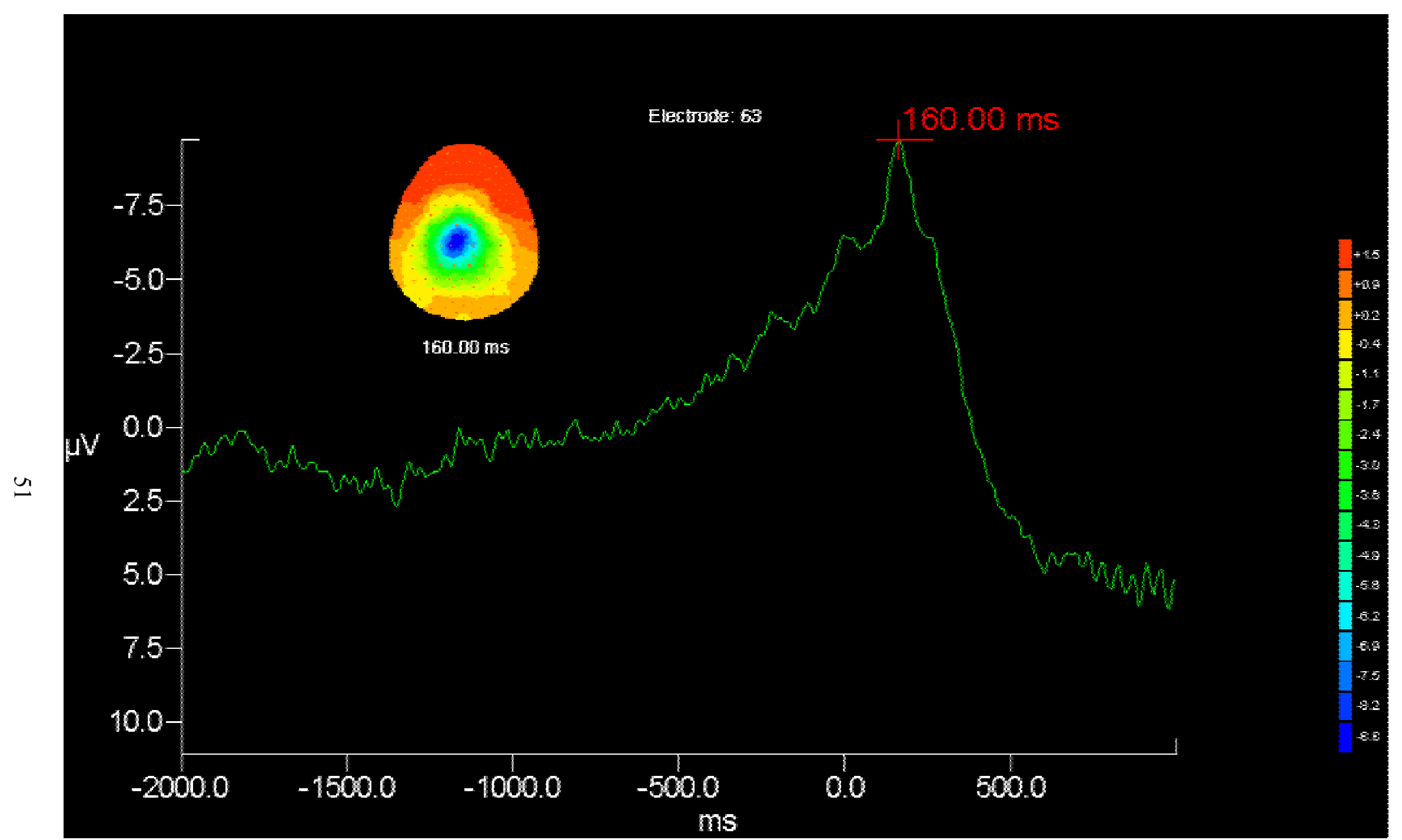

Figure 3.1. An averaged epoch with a motor potential map in a paraplegic patient. 


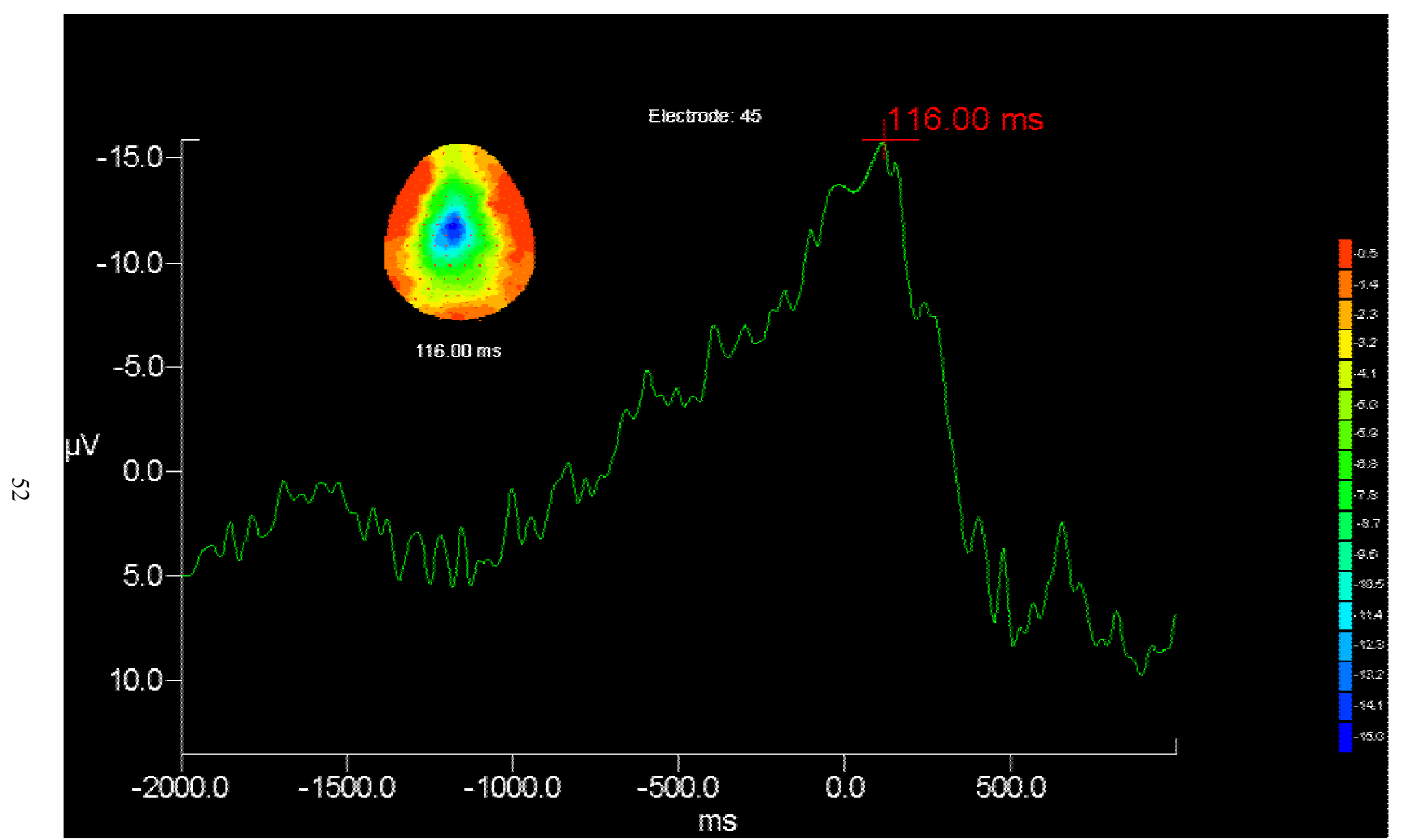

Figure 3.2. An averaged epoch with a motor potential map in a normal control subject. 
waveforms represents the EEG electrode where the motor potential had its largest amplitude. These electrodes are number 63 and number 45 for the two cases shown. It should also be noted that the latency of the motor potential peak is longer in the paraplegic patient recording compared with that of the normal control subject. The figures also show color-coded motor potential maps for the time instant specified on the waveforms. The blue area on the maps represents the location where the motor potential had its maximal amplitude. Figure 3.3 demonstrates a 121-electrode cap layout with averaged epochs shown in miniature for each EEG channel.

Let us assume that $\mathrm{x}$ and $\mathrm{y}$ coordinates of the sites of activation on the scalp are normally distributed in the groups being compared, i.e. normal control subjects, paraplegic and tetraplegic patients. Tables 3.1 through 3.4 show normalized $\mathrm{x}$ and y coordinates of the sites of activation, along with the angles measured from the positive y-axis in the counterclockwise direction. Mean values and standard deviations are shown at the bottom for each test performed. Figures 3.4 and 3.5 schematically show the locations of active areas on a normalized circle of radius 1 representing the head. We want to test the hypothesis whether mean values of $\mathrm{x}$ and $\mathrm{y}$ coordinates are different in the groups under consideration.

Having mean values and variances of the coordinates, it is necessary first to perform a significance test to determine if the underlying variances are equal, that is, we want to test the hypothesis $H_{0}: \sigma_{1}^{2}=\sigma_{2}^{2}$ versus $H_{1}: \sigma_{1}^{2} \neq \sigma_{2}^{2}$.

The results of the significance test for the equality of variances indicate which of the two $t$-tests is to be used for subsequent analysis of the equality of mean values. 


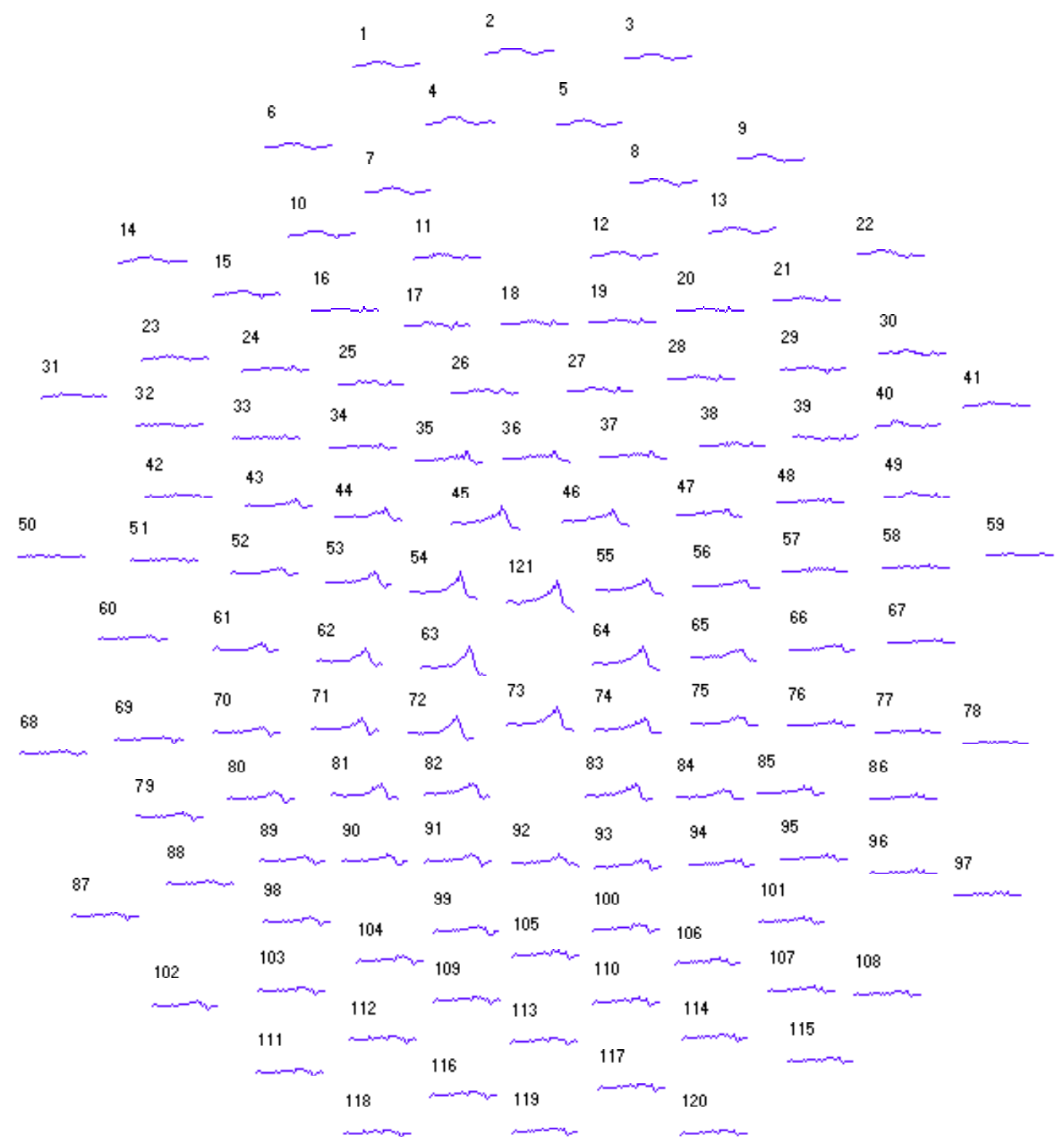

Figure 3.3. A 121-electrode cap layout with averaged epochs. 
Table 3.1. Control subjects: Normalized coordinates and angle values in the motor potential localization study.

\begin{tabular}{|c|c|c|c|c|c|c|c|c|c|c|c|c|c|}
\hline \multirow{2}{*}{\multicolumn{2}{|c|}{ Controls }} & \multicolumn{2}{|c|}{ LFSP } & \multicolumn{2}{|c|}{ RFSP } & \multicolumn{2}{|c|}{$\begin{array}{l}\text { LFPM } \\
\end{array}$} & \multicolumn{2}{|c|}{ RFPM } & \multicolumn{4}{|c|}{ Angle from the positive $y$-axis, degrees } \\
\hline & & abscissa & ordinate & abscissa & ordinate & abscissa & ordinate & abscissa & ordinate & LFSP & RFSP & LFPM & RFPM \\
\hline 1 & D. I. & 0.198 & 0.353 & -0.119 & 0.294 & 0.696 & 0.04 & -0.13 & 0.056 & 29.28835 & -22.0362 & 86.71076 & -66.6951 \\
\hline 3 & V. A. & 0.73 & 0.228 & -0.24 & 0.483 & 0.637 & 0.217 & -0.42 & 0.237 & 72.65491 & -26.4225 & 71.18811 & -60.5646 \\
\hline 4 & G. J. & 0.592 & 0.348 & -0.231 & 0.317 & & & & & 59.55138 & -36.0811 & & \\
\hline 5 & S. L. & 0.257 & 0.181 & -0.327 & 0.07 & 0.51 & 0.034 & -0.559 & 0.065 & 54.84372 & -77.9172 & 86.18593 & -83.3675 \\
\hline 7 & R. F. & & & & & 0.375 & 0.067 & & & & & 79.87004 & \\
\hline 8 & J. H. & 0.311 & 0.258 & -0.597 & 0.098 & 0.381 & 0.01 & -0.131 & 0.232 & 50.32151 & -80.6778 & 88.49652 & -29.4515 \\
\hline 9 & V. K. & 0.469 & 0.417 & -0.45 & 0.413 & 0.802 & 0.238 & -0.855 & 0.299 & 48.35888 & -47.455 & 73.47132 & -70.7249 \\
\hline 10 & K. D. & & & -0.324 & 0.595 & & & -0.343 & 0.483 & & -28.57 & & -35.3803 \\
\hline 11 & W. J. F. & & & -0.133 & 0.584 & & & -0.397 & 0.639 & & -12.8297 & & -31.852 \\
\hline 15 & S. W. & & & -0.482 & 0.465 & & & & & & -46.0284 & & \\
\hline & Mean & 0.4155 & 0.256 & -0.35915 & 0.343231 & 0.608286 & 0.111857 & -0.3953 & 0.3249 & 56.97429 & -45.3694 & 80.6076 & -49.5287 \\
\hline & Std dev. & 0.182692 & 0.107764 & 0.185236 & 0.177948 & 0.193065 & 0.095588 & 0.233871 & 0.184931 & 14.75246 & 22.75272 & 6.782129 & 19.99566 \\
\hline
\end{tabular}


Table 3.2. Paraplegic patients: Normalized coordinates and angle values in the motor potential localization study.

\begin{tabular}{|c|c|c|c|c|c|c|c|c|c|c|c|c|c|}
\hline \multirow{2}{*}{\multicolumn{2}{|c|}{$\begin{array}{c}\text { Paraplegic } \\
\text { patients }\end{array}$}} & \multicolumn{2}{|c|}{ LFSP } & \multicolumn{2}{|c|}{ RFSP } & \multicolumn{2}{|c|}{ LFPM } & \multicolumn{2}{|c|}{ RFPM } & \multicolumn{4}{|c|}{ Angle from the positive $y$-axis, degrees } \\
\hline & & abscissa & ordinate & abscissa & ordinate & abscissa & ordinate & abscissa & ordinate & LFSP & RFSP & LFPM & RFPM \\
\hline 1 & M. C. & & & -0.207 & 0.067 & & & & & & -72.0647 & & \\
\hline 2 & L. N. & & & -0.093 & -0.097 & & & & & & -136.206 & & \\
\hline 3 & G. J. & & & -0.084 & 0.162 & & & & & & -27.4076 & & \\
\hline 4 & J. R. & 0.44 & -0.202 & & & 0.879 & -0.191 & & & 114.6594 & & 102.2594 & \\
\hline 5 & D. S. & 0.841 & 0.137 & -0.498 & 0.017 & 0.659 & 0.174 & -0.401 & 0.117 & 80.74771 & -88.0449 & 75.20937 & -73.7343 \\
\hline 6 & M. S. & 0.797 & -0.03 & -0.186 & -0.04 & 0.922 & -0.074 & & & 92.15566 & -102.137 & 94.58874 & \\
\hline 7 & W. B. & 0.237 & 0.22 & -0.365 & -0.161 & 0.716 & 0.151 & -0.452 & -0.106 & 47.13037 & -113.802 & 78.09118 & -103.198 \\
\hline 8 & H. M. & 0.389 & 0.16 & -0.021 & 0.045 & 0.633 & -0.383 & & & 67.64209 & -25.0169 & 121.1763 & \\
\hline 9 & H. L. & 0.325 & 0.104 & -0.126 & 0.116 & & & & & 72.25533 & -47.3662 & & \\
\hline 10 & F. D. & 0.21 & 0.013 & & & & & & & 86.45764 & & & \\
\hline 11 & B. D. & & & -0.433 & 0.151 & & & -0.702 & 0.046 & & -70.7748 & & -86.2509 \\
\hline 12 & H. R. & 0.845 & 0.16 & -0.321 & 0.096 & 0.83 & 0.017 & -0.167 & -0.053 & 79.27803 & -73.3499 & 88.82664 & -107.608 \\
\hline 13 & B. T. & & & & & & & -0.226 & 0.131 & & & & -59.9014 \\
\hline & Mean & 0.5105 & 0.07025 & -0.2334 & 0.0356 & 0.773167 & -0.051 & -0.3896 & 0.027 & 80.04078 & -75.617 & 93.35859 & -86.1385 \\
\hline & Std dev. & 0.273195 & 0.137101 & 0.162094 & 0.106863 & 0.120442 & 0.212794 & 0.210911 & 0.104123 & 19.58467 & 36.07202 & 16.95633 & 19.9638 \\
\hline
\end{tabular}


Table 3.3. Tetraplegic patients: Normalized coordinates and angle values in the motor potential localization study.

\begin{tabular}{|c|c|c|c|c|c|c|c|c|c|c|c|c|c|}
\hline \multirow{2}{*}{\multicolumn{2}{|c|}{$\begin{array}{c}\text { Tetraplegic } \\
\text { patients }\end{array}$}} & \multicolumn{2}{|c|}{$\overline{\text { LFSP }}$} & \multicolumn{2}{|c|}{ RFSP } & \multicolumn{2}{|c|}{ LFPM } & \multicolumn{2}{|c|}{ RFPM } & \multicolumn{4}{|c|}{ Angle from the positive $y$-axis, degrees } \\
\hline & & \multirow{2}{*}{$\begin{array}{c}\text { abscissa } \\
0.491\end{array}$} & \multirow{2}{*}{$\begin{array}{c}\text { ordinate } \\
0.105\end{array}$} & \multirow{2}{*}{$\begin{array}{c}\text { abscissa } \\
-0.331\end{array}$} & \multirow{2}{*}{$\begin{array}{c}\text { ordinate } \\
-0.145\end{array}$} & \multirow[t]{2}{*}{ abscissa } & ordinate & abscissa & ordinate & \multirow{2}{*}{$\begin{array}{c}\text { LFSP } \\
77.92915\end{array}$} & \multirow{2}{*}{$\begin{array}{c}\text { RFSP } \\
-113.657\end{array}$} & \multirow[t]{2}{*}{ LFPM } & \multirow[t]{2}{*}{ RFPM } \\
\hline 1 & P. M. & & & & & & & & & & & & \\
\hline 2 & J. R. & & & -0.197 & 0.051 & 0.307 & -0.134 & -0.242 & 0.122 & & -80.9229 & 113.5804 & -63.2459 \\
\hline 3 & W. J. & & & & & 0.804 & -0.434 & & & & & 118.3602 & \\
\hline 4 & S. D. & & & -0.185 & 0.159 & & & & & & -49.3223 & & \\
\hline 5 & B. W. & & & & & & & -0.319 & -0.085 & & & & -104.92 \\
\hline 6 & P. C. & 0.219 & -0.122 & -0.251 & -0.091 & 0.673 & -0.267 & -0.742 & -0.251 & 119.1212 & -109.928 & 111.6398 & -108.689 \\
\hline 7 & B. D. & & & & & 0.642 & -0.05 & & & & & 94.4533 & \\
\hline 8 & B. G. & 0.642 & -0.105 & -0.402 & 0.049 & & & & & 99.28857 & -83.0505 & & \\
\hline 9 & M. J. & 0.315 & 0.074 & -0.572 & -0.34 & 0.61 & 0.055 & -0.79 & -0.184 & 76.77976 & -120.727 & 84.84792 & -103.111 \\
\hline 10 & M. B. & & & -0.771 & 0.03 & & & -0.518 & 0.072 & & -87.7717 & & -82.0868 \\
\hline 11 & S. F. & 0.477 & -0.017 & -0.347 & -0.009 & & & & & 92.04112 & -91.4857 & & \\
\hline & Mean & 0.4288 & -0.013 & -0.382 & 0.037 & 0.6072 & -0.166 & -0.5222 & -0.0652 & 93.03196 & -92.1082 & 104.5763 & -92.4107 \\
\hline & Std dev. & 0.164782 & 0.102291 & 0.200657 & 0.153631 & 0.183302 & 0.190621 & 0.244872 & 0.160386 & 17.41411 & 22.85959 & 14.25348 & 19.32067 \\
\hline
\end{tabular}


Table 3.4. SCI patients: Normalized coordinates and angle values in the motor potential localization study.

\begin{tabular}{|c|c|c|c|c|c|c|c|c|c|c|c|c|c|}
\hline \multirow{2}{*}{\multicolumn{2}{|c|}{$\begin{array}{c}\text { SCI } \\
\text { patients }\end{array}$}} & \multicolumn{2}{|c|}{ LFSP } & \multicolumn{2}{|c|}{ RFSP } & \multicolumn{2}{|c|}{ LFPM } & \multicolumn{2}{|c|}{ RFPM } & \multicolumn{4}{|c|}{ Angle from the positive y-axis, degrees } \\
\hline & & abscissa & ordinate & abscissa & ordinate & abscissa & ordinate & abscissa & ordinate & LFSP & RFSP & LFPM & RFPM \\
\hline 2 & L. N. & & & -0.093 & -0.097 & & & & & & -136.206 & & \\
\hline 4 & J. R. & 0.44 & -0.202 & & & 0.879 & -0.191 & & & 114.6594 & & 102.2594 & \\
\hline 5 & D. S. & 0.841 & 0.137 & -0.498 & 0.017 & 0.659 & 0.174 & -0.401 & 0.117 & 80.74771 & -88.0449 & 75.20937 & -73.7343 \\
\hline 6 & M. S. & 0.797 & -0.03 & -0.186 & -0.04 & 0.922 & -0.074 & & & 92.15566 & -102.137 & 94.58874 & \\
\hline 7 & W. B. & 0.237 & 0.22 & -0.365 & -0.161 & 0.716 & 0.151 & -0.452 & -0.106 & 47.13037 & -113.802 & 78.09118 & -103.198 \\
\hline 10 & F. D. & 0.21 & 0.013 & & & & & & & 86.45764 & & & \\
\hline 11 & B. D. & & & -0.433 & 0.151 & & & -0.702 & 0.046 & & -70.7748 & & -86.2509 \\
\hline 12 & H. R. & 0.845 & 0.16 & -0.321 & 0.096 & 0.83 & 0.017 & -0.167 & -0.053 & 79.27803 & -73.3499 & 88.82664 & -107.608 \\
\hline 13 & B. T. & & & & & & & -0.226 & 0.131 & & & & -59.9014 \\
\hline 14 & P. M. & 0.491 & 0.105 & -0.331 & -0.145 & & & & & 77.92915 & -113.657 & & \\
\hline 15 & J. R. & & & -0.197 & 0.051 & 0.307 & -0.134 & -0.242 & 0.122 & & -80.9229 & 113.5804 & -63.2459 \\
\hline 20 & B. D. & & & & & 0.642 & -0.05 & & & & & 94.4533 & \\
\hline 21 & B. G. & 0.642 & -0.105 & -0.402 & 0.049 & & & & & 99.28857 & -83.0505 & & \\
\hline 22 & M. J. & 0.315 & 0.074 & -0.572 & -0.34 & 0.61 & 0.055 & -0.79 & -0.184 & 76.77976 & -120.727 & 84.84792 & -103.111 \\
\hline 23 & M. B. & & & -0.771 & 0.03 & & & -0.518 & 0.072 & & -87.7717 & & -82.0868 \\
\hline 24 & S. F. & 0.477 & -0.017 & -0.347 & -0.009 & & & & & 92.04112 & -91.4857 & & \\
\hline & Mean & 0.479077 & 0.038231 & -0.299444 & 0.003333 & 0.697727 & -0.10327 & -0.4559 & -0.0191 & 85.03739 & -82.9464 & 98.45756 & -89.2746 \\
\hline & td dev. & 0.233023 & 0.127396 & 0.190426 & 0.130929 & 0.167944 & 0.201946 & 0.226505 & 0.136428 & 19.18596 & 31.2272 & 16.10412 & 18.81406 \\
\hline
\end{tabular}

The table shows normalized coordinates of the location of the motor potential (MP) on the scalp. The difference in the locations is statistically significant in the anterior-posterior direction for control subjects and SCI patients ( $p$-value less than 0.05). 


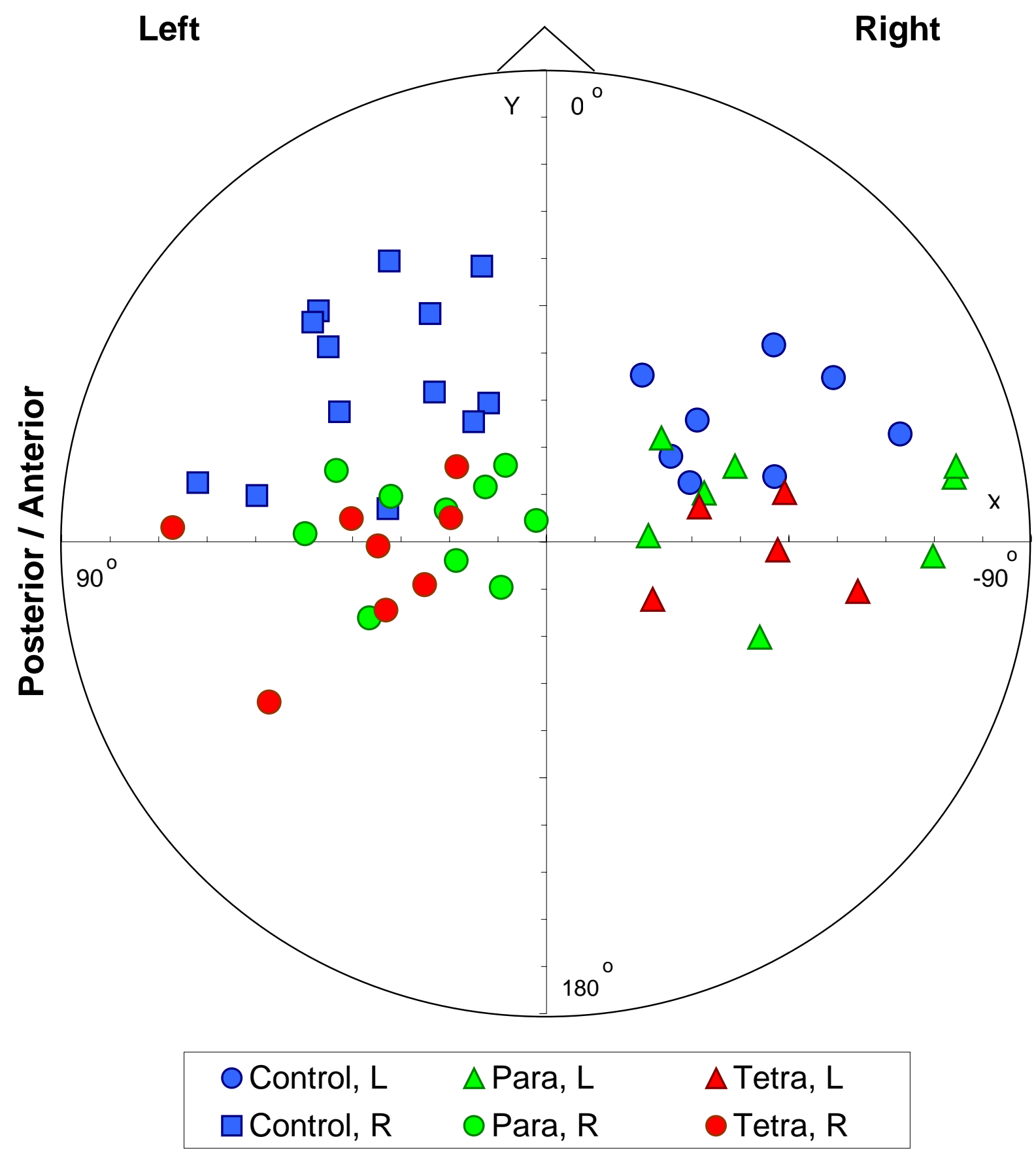

Figure 3.4. Mapping of motor potentials in left and right finger self-paced movements. 


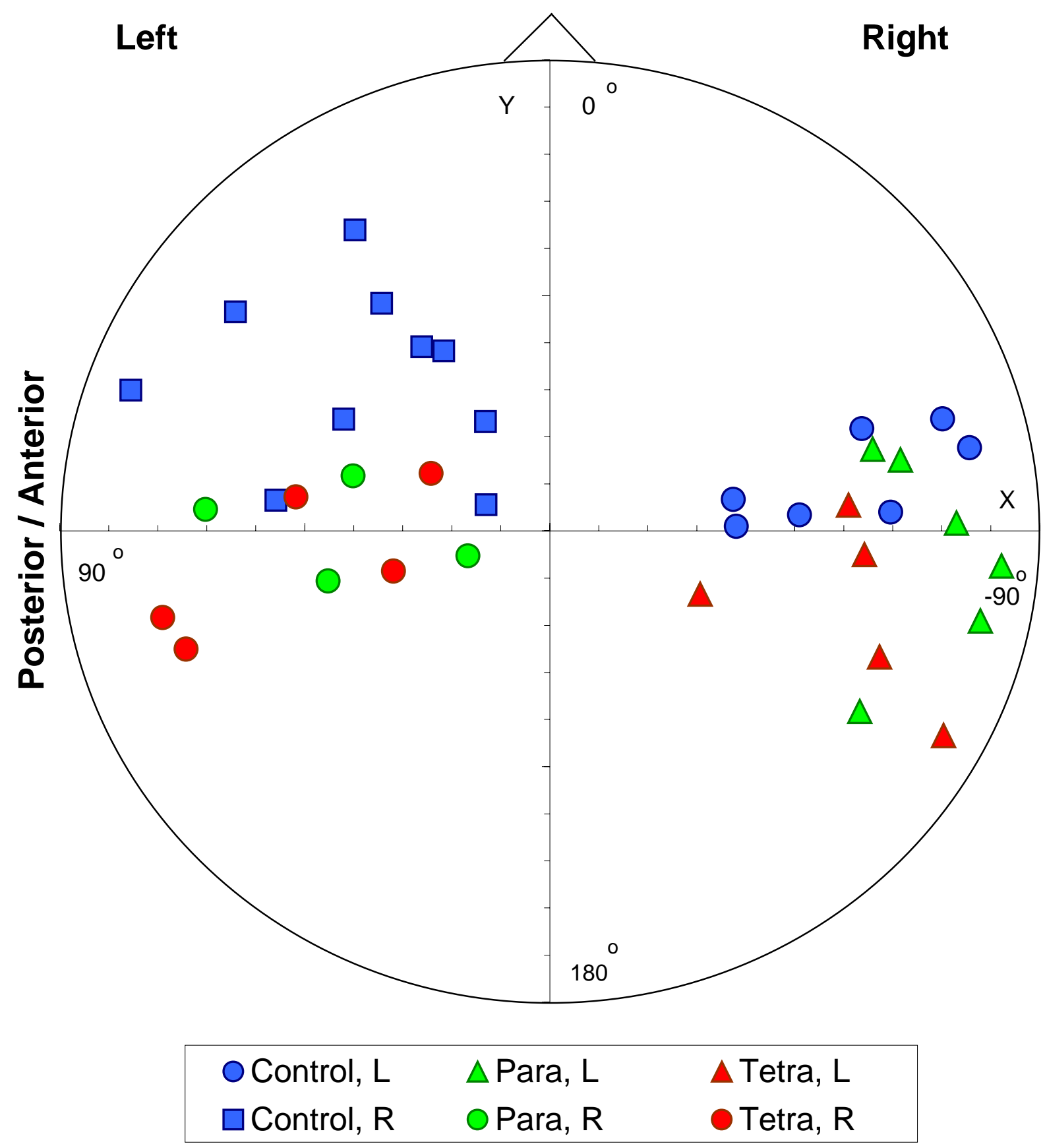

Figure 3.5. Mapping of motor potentials in left and right finger passive movements. 


\subsubsection{Motor potential distribution: the F test}

Prior to analyzing statistically the distribution of the sites of activation, it is necessary to perform the significance test for the equality of variances for every pair of groups to be analyzed. The $F$ test has been performed in pairs for the following groups: control subjects and paraplegic patients, control subjects and tetraplegic patients, and control subjects and SCI patients. The SCI group includes individuals from both paraplegic and tetraplegic group. Comparison of the variances of the distribution of motor potentials along the $\mathrm{x}$ and $\mathrm{y}$ axes, as well as the angle thereof measured from the positive y-axis in the counterclockwise direction is the object of this test. Tables 3.5 through 3.7 show the results of the $F$ test. It can be seen that the variances of the distribution of motor potentials are not statistically different (with the probability of a type I error equal to 0.05 ) for all tests, except for the variances of the angle distribution in the left finger passive movement test. The words "accepted" and "rejected" refer to the acceptance and rejection of the null hypothesis $\mathrm{H}_{0}$, respectively.

\subsubsection{Motor potential distribution: the t-tests}

Having performed the $F$ test on the data, we can proceed with significance testing for the equality of means of the $\mathrm{x}, \mathrm{y}$ and angle distribution of motor potentials. As seen from Table 3.8, there is no statistical significance in data variances of any pair of parameters in the self-paced movement tests. Therefore, the $t$-test assuming equal variances is to be performed, as discussed in the previous chapter. In the passive movement tests, however, the variances of the angle 
Table 3.5. Controls and paraplegics: $F$ test for the equality of two variances.

\begin{tabular}{|lllll|}
\hline \multicolumn{1}{c}{ LFSP } & \multicolumn{1}{c}{ RFSP } & \multicolumn{1}{c|}{ LFPM } & \multicolumn{1}{c|}{ RFPM } \\
\hline Upper limit & $\mathrm{F}_{7,7,975}=4.99$ & $\mathrm{~F}_{12,9,975}=3.87$ & $\mathrm{~F}_{6,5,975}=6.98$ & $\mathrm{~F}_{9,4,975}=8.90$ \\
Lower limit & $\mathrm{F}_{7,7,025}=0.200$ & $\mathrm{~F}_{12,9,025}=0.292$ & $\mathrm{~F}_{6,5,025}=0.167$ & $\mathrm{~F}_{9,4,025}=0.212$ \\
& & & & \\
Variance norm, $\mathrm{x}$ & 0.03338 & 0.03431 & 0.03727 & 0.05470 \\
Variance para, $\mathrm{x}$ & 0.07464 & 0.02627 & 0.01451 & 0.04448 \\
F statistic & 2.23618 & 1.30592 & 2.56952 & 1.22958 \\
Hypothesis Ho & accepted & accepted & accepted & accepted \\
& & & & \\
Variance norm, y & 0.01161 & 0.03167 & 0.00914 & 0.03420 \\
Variance para, y & 0.01880 & 0.01142 & 0.04528 & 0.01084 \\
F statistic & 1.61858 & 2.77290 & 0.20179 & 3.15449 \\
Hypothesis Ho & accepted & accepted & accepted & accepted \\
& & & & \\
Variance norm, angle & 217.63494 & 517.68620 & 45.99727 & 399.82627 \\
Variance para, angle & 383.55919 & 1301.19067 & 287.51724 & 398.55346 \\
F statistic & 1.76240 & 0.39786 & 0.15998 & 1.00319 \\
Hypothesis Ho & accepted & accepted & rejected & accepted \\
\hline
\end{tabular}

Table 3.6. Controls and tetraplegics: $F$ test for the equality of two variances.

\begin{tabular}{|lllll|}
\hline \multicolumn{1}{c}{ LFSP } & \multicolumn{1}{c}{ RFSP } & \multicolumn{1}{c|}{ LFPM } & \multicolumn{1}{c|}{ RFPM } \\
\hline Upper limit & $\mathrm{F}_{7,4.975}=9.07$ & $\mathrm{~F}_{7,12,975}=3.61$ & $\mathrm{~F}_{6,4,975}=9.20$ & $\mathrm{~F}_{9,4,975}=8.90$ \\
Lower limit & $\mathrm{F}_{7,4.025}=0.181$ & $\mathrm{~F}_{7,12,025}=0.214$ & $\mathrm{~F}_{6,4,025}=0.161$ & $\mathrm{~F}_{9,4,025}=0.212$ \\
& & & & \\
Variance norm, $\mathrm{x}$ & 0.033376 & 0.034312 & 0.037274 & 0.054696 \\
Variance tetra, $\mathrm{x}$ & 0.027153 & 0.040263 & 0.0336 & 0.059962 \\
F statistic & 1.229184 & 1.173426 & 1.109352 & 0.912171 \\
Hypothesis Ho & accepted & accepted & accepted & accepted \\
& & & & \\
Variance norm, y & 0.011613 & 0.031665 & 0.009137 & 0.034199 \\
Variance tetra, y & 0.010464 & 0.023603 & 0.036337 & 0.025724 \\
F statistic & 1.109872 & 0.745375 & 0.251459 & 1.329491 \\
Hypothesis Ho & accepted & accepted & accepted & accepted \\
& & & & \\
Variance norm, angle & 217.6349 & 517.6862 & 45.99727 & 399.8263 \\
Variance tetra, angle & 303.2511 & 543.6325 & 203.1618 & 373.2884 \\
F statistic & 0.717672 & 1.05012 & 0.226407 & 1.071092 \\
Hypothesis Ho & accepted & accepted & accepted & accepted \\
\hline
\end{tabular}


Table 3.7. Controls and SCI patients: $F$ test for the equality of two variances.

\begin{tabular}{|lllll|}
\hline \multicolumn{1}{c}{ LFSP } & \multicolumn{1}{c}{ RFSP } & \multicolumn{1}{c|}{ LFPM } & \multicolumn{1}{c|}{ RFPM } \\
\hline Upper limit & $\mathrm{F}_{7,12.975}=3.61$ & $\mathrm{~F}_{12,17,975}=2.81$ & $\mathrm{~F}_{6,10,975}=4.07$ & $\mathrm{~F}_{9,9,975}=4.02$ \\
Lower limit & $\mathrm{F}_{7,12.025}=0.214$ & $\mathrm{~F}_{12,17,025}=0.321$ & $\mathrm{~F}_{6,10,025}=0.182$ & $\mathrm{~F}_{9,9,025}=0.249$ \\
& & & & \\
Variance norm, $\mathrm{x}$ & 0.033376 & 0.034312 & 0.037274 & 0.054696 \\
Variance SCI, $\mathrm{x}$ & 0.0543 & 0.036262 & 0.028205 & 0.051304 \\
F statistic & 0.614666 & 0.946237 & 1.321525 & 1.066105 \\
Hypothesis Ho & accepted & accepted & accepted & accepted \\
& & & & \\
Variance norm, y & 0.011613 & 0.031665 & 0.009137 & 0.034199 \\
Variance SCI, y & 0.01623 & 0.017142 & 0.040782 & 0.018613 \\
F statistic & 0.715549 & 1.8472 & 0.224048 & 1.83744 \\
Hypothesis Ho & accepted & accepted & accepted & accepted \\
& & & & \\
Variance norm, angle & 217.6349 & 517.6862 & 45.99727 & 399.8263 \\
Variance SCI, angle & 368.1011 & 978.0749 & 259.3426 & 353.9687 \\
F statistic & 0.591237 & 0.529291 & 0.177361 & 1.129553 \\
Hypothesis Ho & accepted & accepted & rejected & accepted \\
\hline
\end{tabular}

Table 3.8. $F$ test for the equality of two variances: MP localization.

\begin{tabular}{|llllll|}
\hline Condition & \multicolumn{5}{c|}{ Control subjects } \\
\hline \multirow{3}{*}{ Paraplegic patients } & Parameter & LFSP & RFSP & LFPM & RFPM \\
& X coordinate & accepted & accepted & accepted & accepted \\
& Y coordinate & accepted & accepted & accepted & accepted \\
& Angle & accepted & accepted & rejected & accepted \\
\hline \multirow{3}{*}{ Tetraplegic patients } & X coordinate & accepted & accepted & accepted & accepted \\
& Y coordinate & accepted & accepted & accepted & accepted \\
& Angle & accepted & accepted & accepted & accepted \\
\hline \multirow{3}{*}{ SCI patients overall } & X coordinate & accepted & accepted & accepted & accepted \\
& Y coordinate & accepted & accepted & accepted & accepted \\
& Angle & accepted & accepted & rejected & accepted \\
\hline
\end{tabular}


distribution in paraplegics and SCI patients overall were statistically different. This necessitates performing $t$-tests assuming unequal variances on these two cases. Tables 3.9 through 3.11 show results of the $t$-tests performed in pairs on control subjects and paraplegic, tetraplegic, and SCI

patients overall, respectively. The tables include the critical values of the confidence interval for each test, the mean values of the variables being tested, the test statistics, $p$-values, and the results of the hypothesis testing. Critical values of the confidence intervals for the two unequal variance cases are also presented in the tables as additional entries. Table 3.12 summarizes the results in a concise form. A strong correlation between condition and the location of motor potentials can be observed. The differences in the MP locations along the $\mathrm{x}$-axis are not significant in any test. However, the distribution of the MP locations along the y-axis as well as the distribution of angles show statistically significant difference in all cases except the left finger passive movement test for paraplegics.

\subsection{Source Localization Results}

Having performed statistical analysis of the data from the motor potential localization study, we proceed with localization of putative sources (current dipoles) responsible for the generation of the potential pattern recorded from the scalp in the EEG tests. A current reconstruction and imaging software package, CURRY v.3.0, was used for the reconstructions with subject-specific MR images to restrict the volume conductor geometry to the individual anatomy. There are important theoretical objections to comparisons of dipole sources across 
Table 3.9. Controls and paraplegic patients: $t$-test for the equality of means.

\begin{tabular}{|lllll|}
\hline & \multicolumn{1}{c}{ LFSP } & \multicolumn{1}{c}{ RFSP } & \multicolumn{1}{c|}{ LFPM } & \multicolumn{1}{c|}{ RFPM } \\
\hline Critical values: & & & & \\
Equal variance case & $\mathrm{t}_{14, .975}=2.14479$ & $\mathrm{t}_{21, .975}=2.07961$ & $\mathrm{t}_{11, .975}=2.20099$ & $\mathrm{t}_{13,975}=2.16037$ \\
Unequal variance case & - & - & $\mathrm{t}_{5,975}=2.57058$ & - \\
Mean norm, $\mathrm{x}$ & & & & \\
Mean para, $\mathrm{x}$ & 0.4155 & -0.35915 & 0.608286 & -0.3953 \\
Test statistic & 0.5105 & -0.2334 & 0.773167 & -0.3896 \\
$p$-value & -0.81759 & -1.70168 & -1.80612 & -0.04583 \\
Hypothesis Ho & 0.42729 & 0.103576 & 0.098307 & 0.964139 \\
& accepted & accepted & accepted & accepted \\
Mean norm, y & & & & \\
Mean para, y & 0.256 & 0.343231 & 0.111857 & 0.3249 \\
Test statistic & 0.07025 & 0.0356 & -0.051 & 0.027 \\
$p$-value & 3.012771 & 4.82371 & 1.830738 & 3.309242 \\
Hypothesis Ho & 0.009313 & $9.1 \mathrm{E}-05$ & 0.094339 & 0.005646 \\
& rejected & rejected & accepted & rejected \\
Mean norm, angle & & & & \\
Mean para, angle & 56.97429 & -45.3694 & 80.6076 & -49.5287 \\
Test statistic & 80.04078 & -75.617 & 33.35859 & -86.1385 \\
$p$-value & -2.66084 & 2.461523 & 1.288876 & 3.344363 \\
Hypothesis Ho & 0.01863 & 0.022574 & 0.253849 & 0.005278 \\
\hline & rejected & rejected & accepted & rejected \\
\hline
\end{tabular}


Table 3.10. Controls and tetraplegic patients: $t$-test for the equality of means.

\begin{tabular}{|lllll|}
\hline & \multicolumn{1}{c}{ LFSP } & \multicolumn{1}{c}{ RFSP } & \multicolumn{1}{c|}{ LFPM } & \multicolumn{1}{c|}{ RFPM } \\
\hline Critical values: & & & & \\
Equal variance case & $\mathrm{t}_{11, .975}=2,20099$ & $\mathrm{t}_{19, .975}=2,09303$ & $\mathrm{t}_{10,975}=2,22814$ & $\mathrm{t}_{13,975}=2,16037$ \\
Unequal variance case & - & - & - & - \\
Mean norm, $\mathrm{x}$ & & & & \\
Mean para, $\mathrm{x}$ & 0.4155 & -0.35915 & 0.608286 & -0.3953 \\
Test statistic & 0.4288 & -0.382 & 0.6072 & -0.5222 \\
$p$-value & -0.13226 & 0.2661 & 0.009799 & 0.976302 \\
Hypothesis Ho & 0.897165 & 0.793028 & 0.992374 & 0.346733 \\
& accepted & accepted & accepted & accepted \\
Mean norm, y & & & & \\
Mean para, y & 0.256 & 0.343231 & 0.111857 & 0.3249 \\
Test statistic & -0.013 & -0.037 & -0.166 & -0.0652 \\
$p$-value & 4.459609 & 4.995193 & 3.354028 & 4.007099 \\
Hypothesis Ho & 0.000963 & $8.04 \mathrm{E}-05$ & 0.007316 & 0.001492 \\
& rejected & rejected & rejected & rejected \\
Mean norm, angle & & & & \\
Mean para, angle & 56.97429 & -45.3694 & 80.6076 & -49.5287 \\
Test statistic & 93.03196 & -91.4285 & 104.5763 & -92.4107 \\
$p$-value & -4.01013 & 4.463921 & -3.92327 & 3.956024 \\
Hypothesis Ho & 0.00205 & 0.000266 & 0.002851 & 0.001643 \\
\hline & rejected & rejected & rejected & rejected \\
\hline
\end{tabular}


Table 3.11. Controls and SCI patients: $t$-test for the equality of means.

\begin{tabular}{|lllll|}
\hline & \multicolumn{1}{c}{ LFSP } & \multicolumn{1}{c}{ RFSP } & \multicolumn{1}{c|}{ LFPM } & RFPM \\
\hline Critical values: & & & & \\
Equal variance case & $\mathrm{t}_{19,975}=2.09302$ & $\mathrm{t}_{29,975}=2.04523$ & $\mathrm{t}_{16,975}=2.11990$ & $\mathrm{t}_{18,975}=2.10092$ \\
Unequal variance case & - & - & $\mathrm{t}_{14, .975}=2.14479$ & - \\
Mean norm, $\mathrm{x}$ & & & & \\
Mean para, $\mathrm{x}$ & 0.4155 & -0.35915 & 0.608286 & -0.3953 \\
Test statistic & 0.479077 & -0.29944 & 0.697727 & -0.4459 \\
$p$-value & -0.65547 & -0.87122 & -1.04055 & 0.588599 \\
Hypothesis Ho & 0.520022 & 0.390788 & 0.313553 & 0.563444 \\
& accepted & accepted & accepted & accepted \\
Mean norm, y & & & & \\
Mean para, y & 0.256 & 0.343231 & 0.111857 & 0.3249 \\
Test statistic & 0.038231 & 0.003333 & -0.10327 & -0.0191 \\
$p$-value & 4.020572 & 6.137364 & 2.616657 & 4.733607 \\
Hypothesis Ho & 0.000731 & $1.09 \mathrm{E}-06$ & 0.018697 & 0.000166 \\
& rejected & rejected & rejected & rejected \\
Mean norm, angle & & & & \\
Mean para, angle & 56.97429 & -45.3694 & 80.6076 & -49.5287 \\
Test statistic & 85.03739 & -82.6443 & 98.45756 & -89.2746 \\
$p$-value & -3.53185 & 3.64922 & -3.25095 & 4.577889 \\
Hypothesis Ho & 0.002228 & 0.001027 & 0.005802 & 0.000233 \\
\hline
\end{tabular}


Table 3.12. $t$-test for the equality of means: MP localization.

\begin{tabular}{|llllll|}
\hline Condition & \multicolumn{5}{c|}{ Control subjects } \\
\hline & Parameter & LFSP & RFSP & LFPM & RFPM \\
\hline \multirow{3}{*}{ Paraplegic patients } & X coordinate & accepted & accepted & accepted & accepted \\
& Y coordinate & rejected & rejected & accepted & rejected \\
& Angle & rejected & rejected & accepted & rejected \\
\hline \multirow{3}{*}{ Tetraplegic patients } & X coordinate & accepted & accepted & accepted & accepted \\
& Y coordinate & rejected & rejected & rejected & rejected \\
& Angle & rejected & rejected & rejected & rejected \\
SCI patients overall & X coordinate & accepted & accepted & accepted & accepted \\
& Y coordinate & rejected & rejected & rejected & rejected \\
& Angle & rejected & rejected & rejected & rejected \\
\hline
\end{tabular}

subjects, namely comparing point sources at different latencies, each of which represents one of an infinite number of possible inverse solutions. The use of dipole source analysis, therefore, has been limited to comparing the motor potential distribution fields with their putative sources in individual subjects. The spatial locations of the underlying dipole sources show the areas in the cortex where the electrophysiological activity took place during the EEG recordings. Because some participants were unable to tolerate the MRI examination due to claustrophobia, dipole source localizations were performed on 22 subjects. Two groups of individuals are compared in this study, control subjects and SCI patients.

Figure 3.6 shows frontal, sagittal and transverse sections of a normal control subject's head with current dipoles shown as arrows. Figure 3.7 shows the dipoles within a brain model rendered from the subject's MR images. The dipoles represent brain areas active during a selfpaced right finger movement test. The larger dipole in the left hemisphere is due to the neuronal activity in the sensorimotor cortex. The two smaller medial dipoles in the intracerebral region are located at the level of the mesial cortex and presumably represent activation of the SMA [28]. 


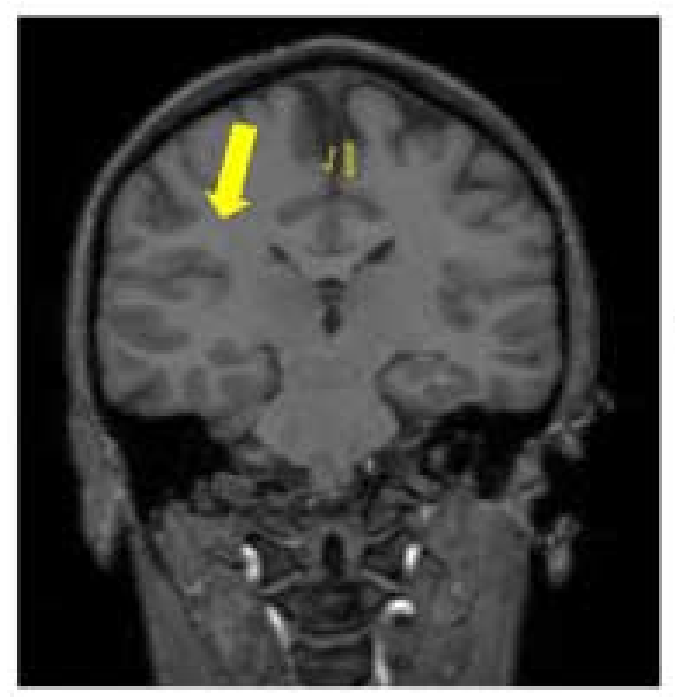

Posterior view

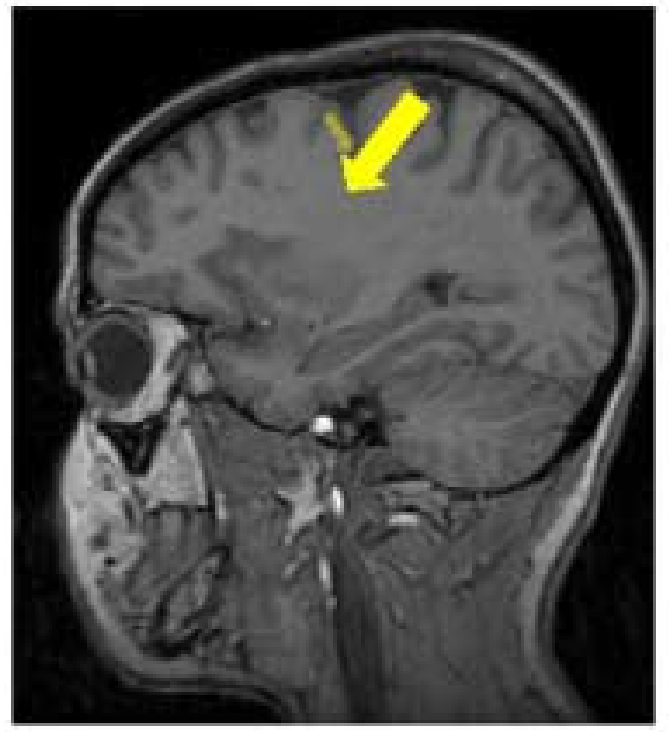

Sagittal view

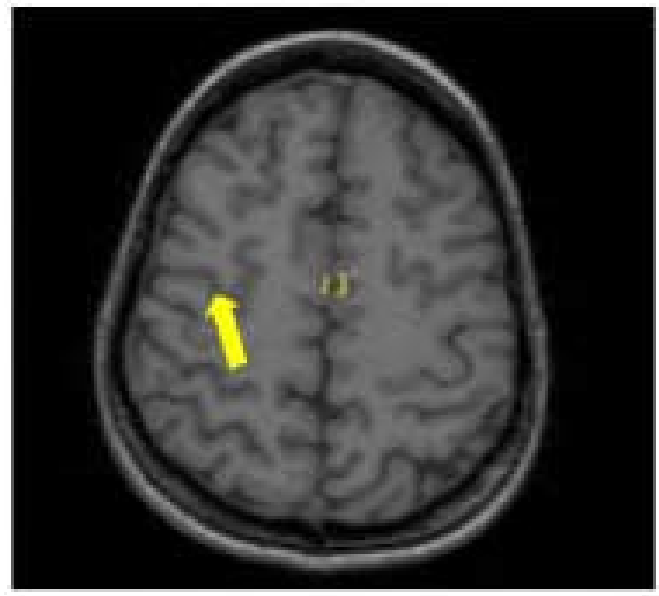

Superior view

Figure 3.6. Sectional views of the source localization results in a control subject, active movement test. 

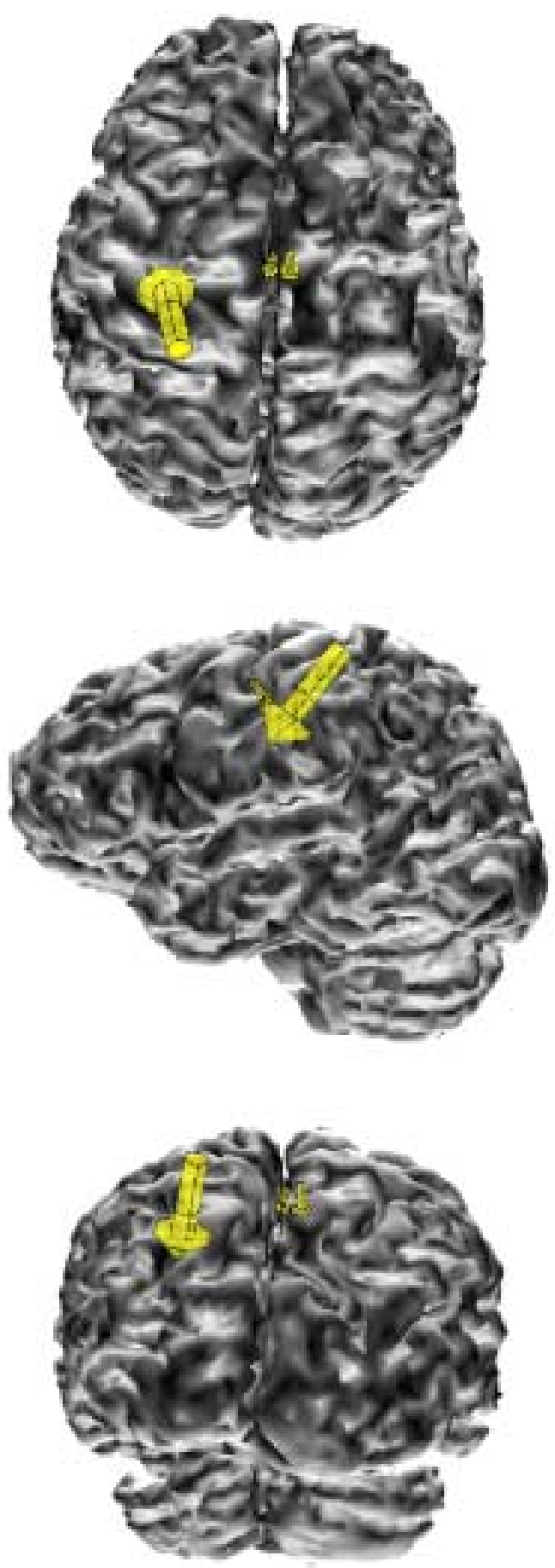

Figure 3.7. Source localization results with a segmented brain model - control subject, active movement test. 
Figure 3.8 and 3.9 show analogous results obtained in a passive movement test from the same individual. The dipole was found only in the contralateral sensorimotor area. Figure 3.10 through 3.13 show dipole localization results obtained from a paraplegic patient in the same kind of tests.

Table 3.13 and 3.14 contain the normalized $\mathrm{x}$ and $\mathrm{y}$-coordinates of the source locations as well as the angles from the positive y-axis thereof found in control subjects and SCI patients in self-paced and passive movement tests respectively. Figures 3.14 and 3.15 demonstrate the source locations on a normalized circle. The distribution of the sources tends to be less spread in this study compared to the motor potential distribution due to the fact that the sources are confined in the brain compartment model, which has a smaller radius than the scalp model. The normalized scalp dimensions were used in both motor potential localization study and source reconstructions to facilitate the comparison of the results of the two studies.

\subsubsection{Results of the F test}

Before comparing the spatial distributions of the sources in the two groups in this study, control subjects and SCI patients, an $F$ test was performed to determine if the variances are equal in any pair of data groups. Table 3.15 shows the results of the $F$ test performed for both selfpaced and passive movement paradigms. The table contains upper and lower limits of the confidence interval for each test, also data variances, values of the test statistic, and the test results with respect to the null hypothesis. The results show that the variances are not statistically different in any pair of data being analyzed (with the probability of a type I error equal to 0.05 ). 


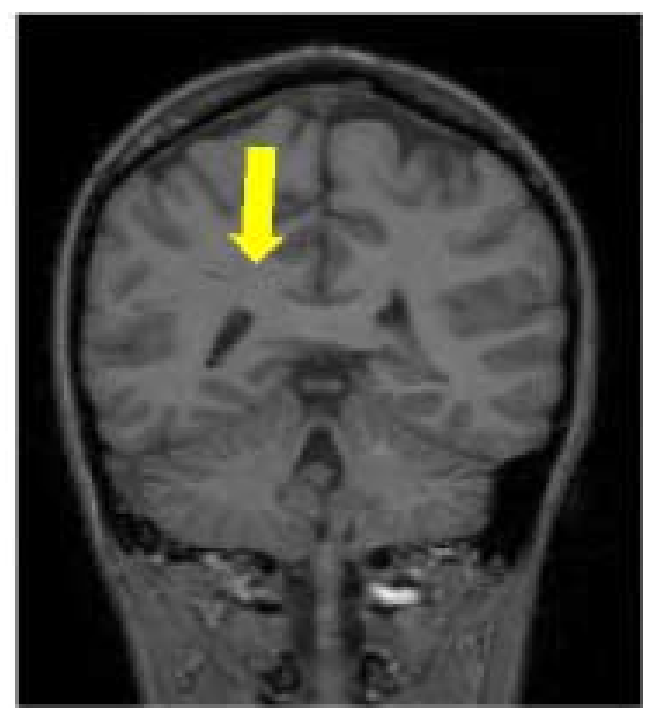

Posterior view

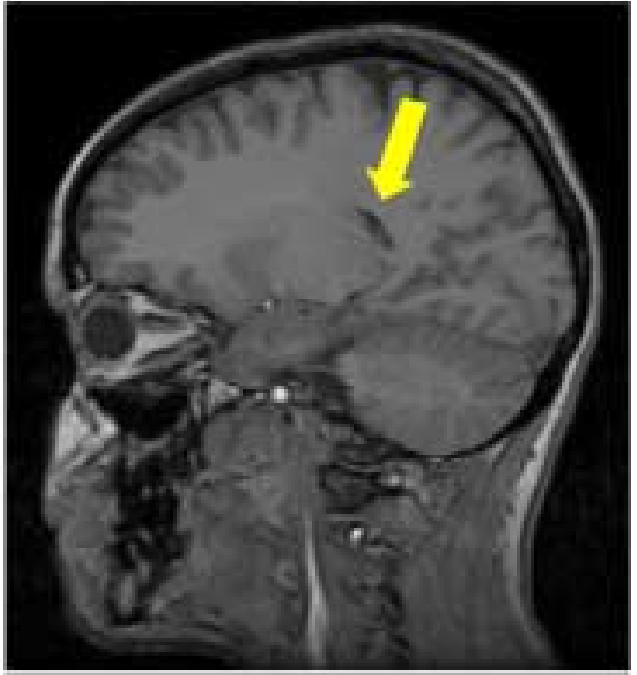

Sagittal view

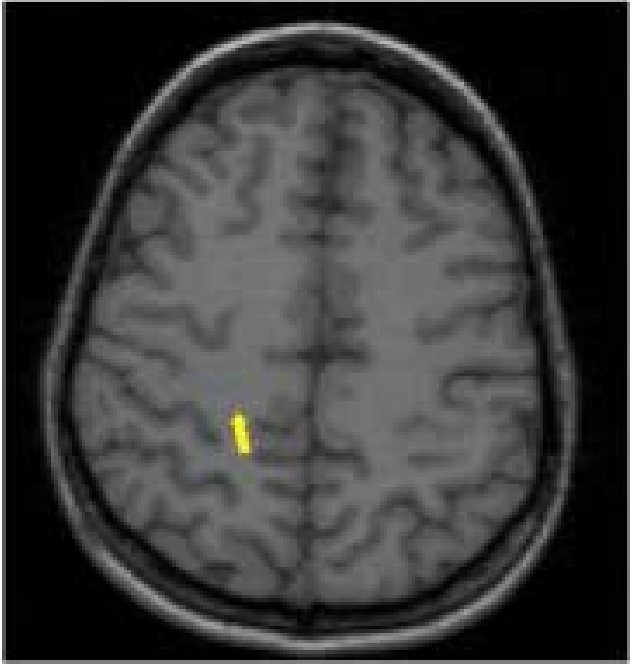

Superior view

Figure 3.8. Sectional views of the source localization results in a control subject, passive movement test. 

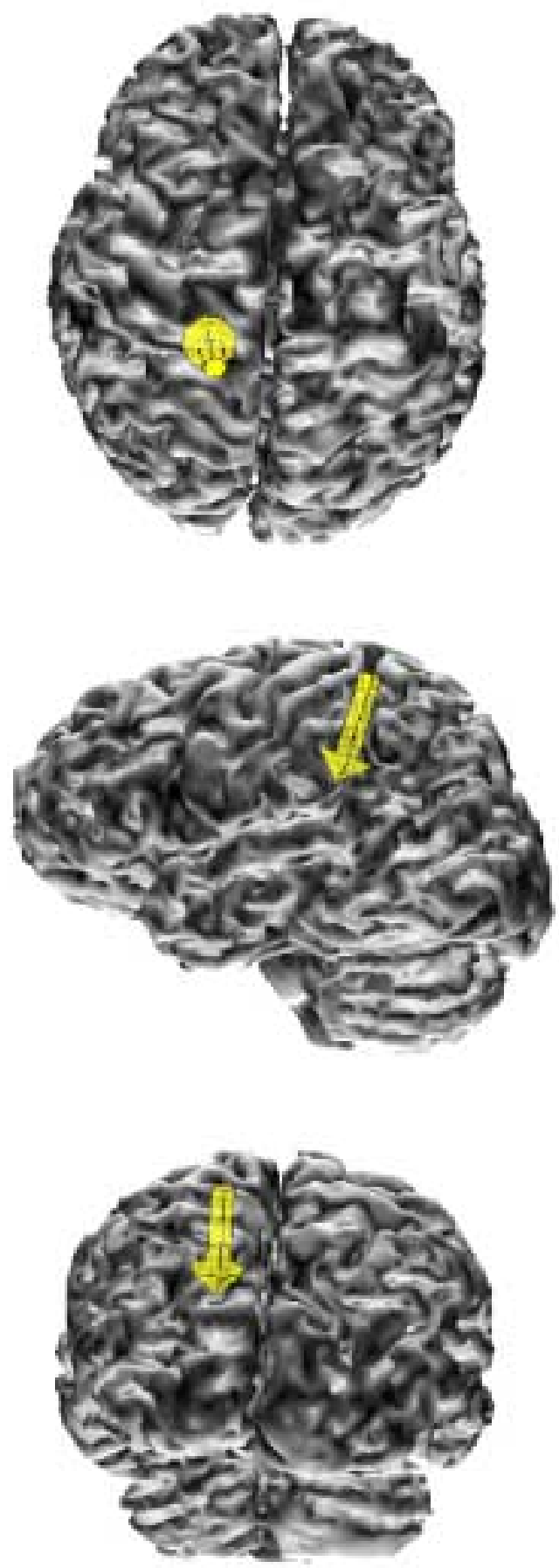

Figure 3.9. Source localization results with a segmented brain model - control subject, passive movement test. 


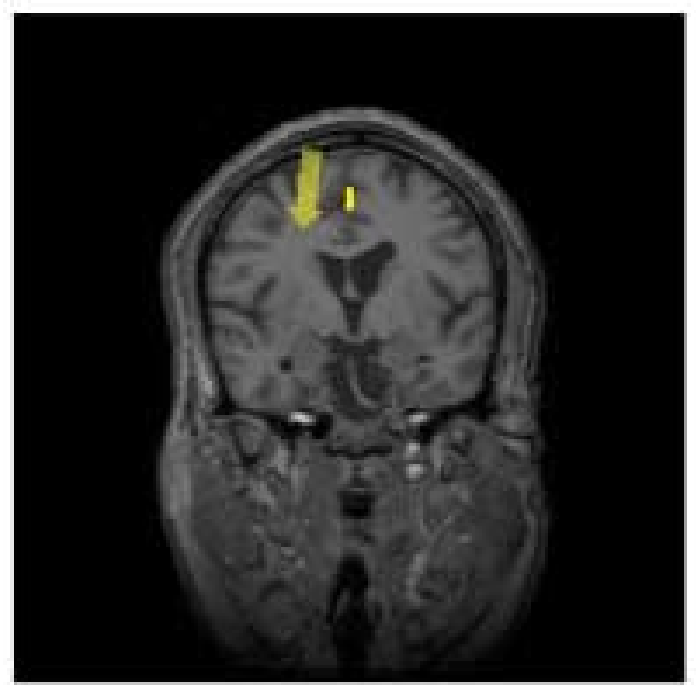

\section{Posterior view}

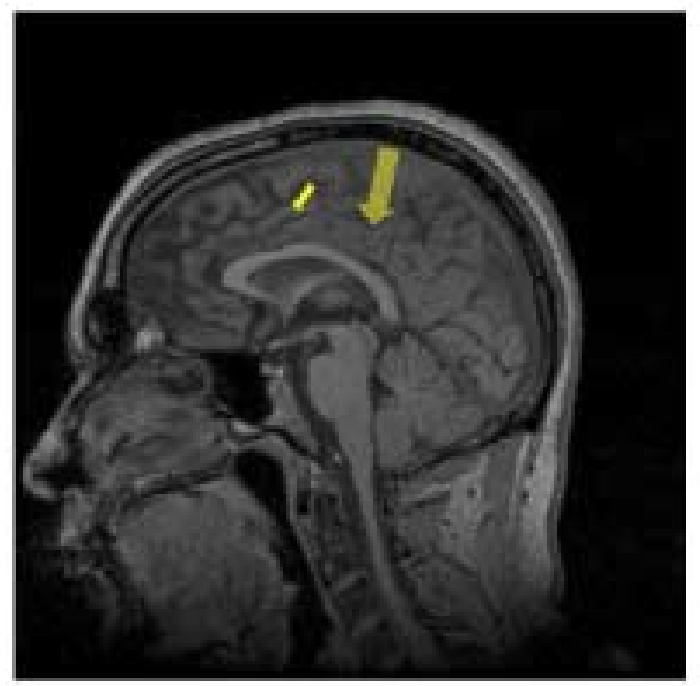

Sagittal view

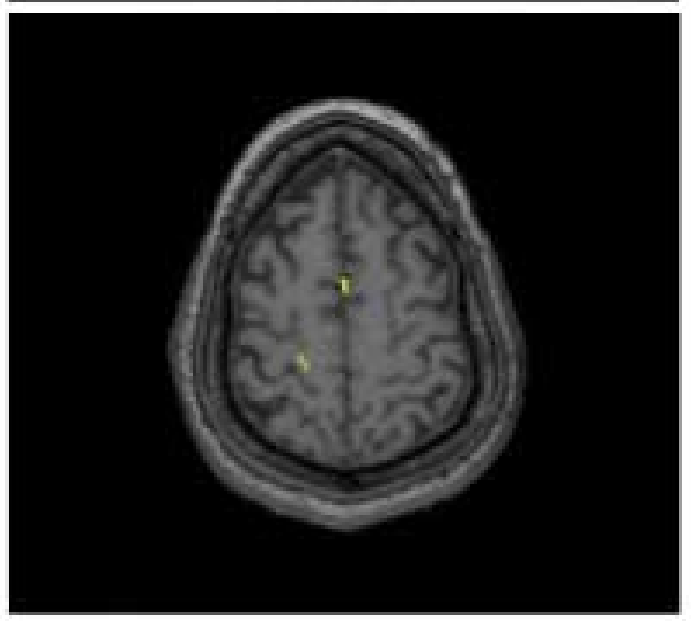

Superior view

Figure 3.10. Sectional views of the source localization results in a paraplegic patient, active movement test. 

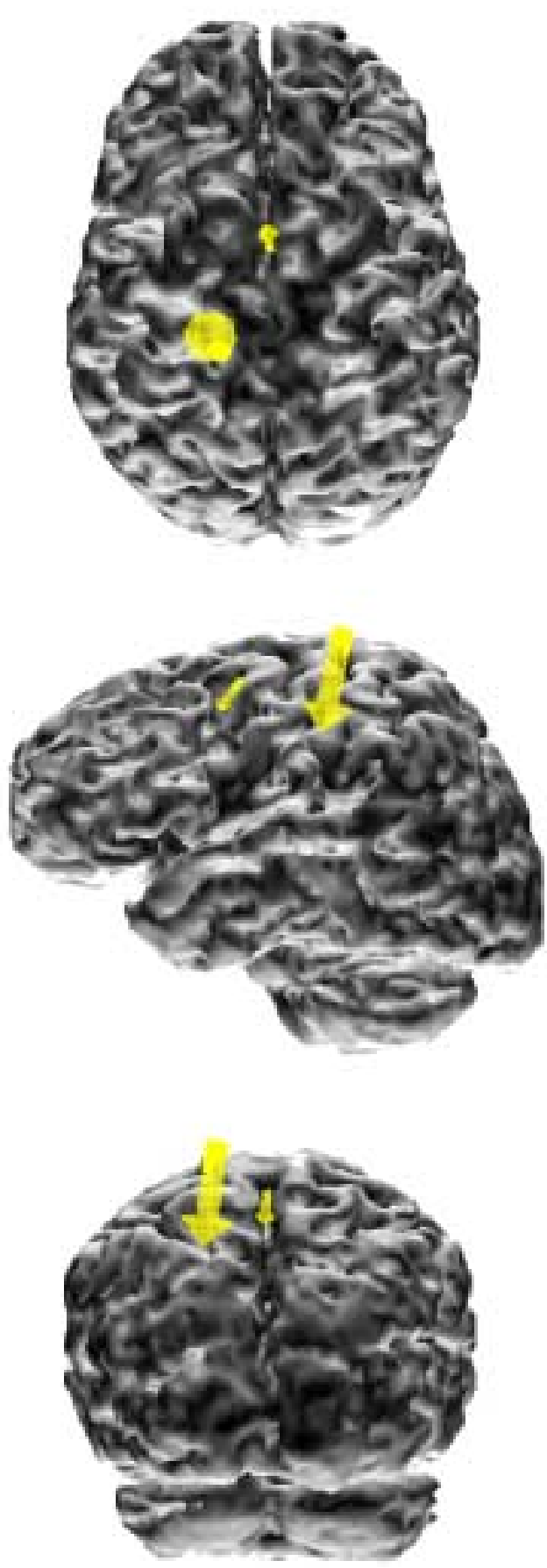

Figure 3.11. Source localization results with a segmented brain model - paraplegic patient, active movement test. 


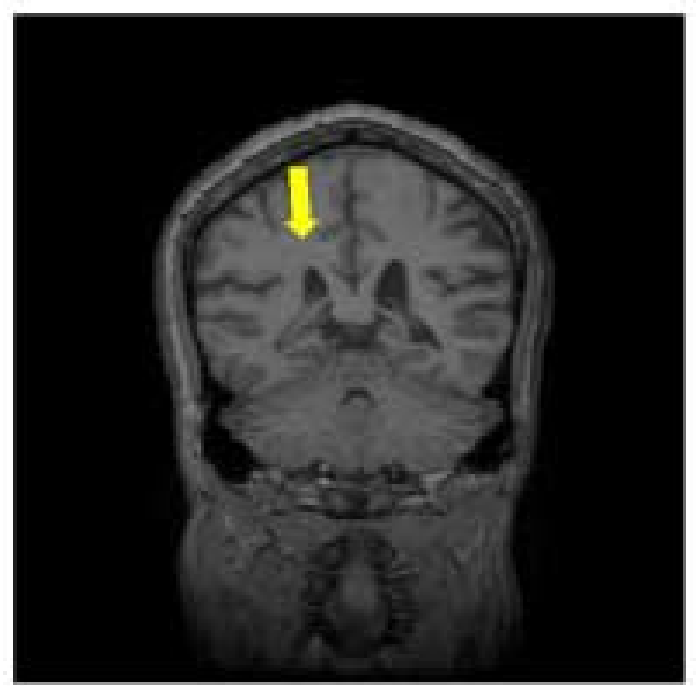

\section{Posterior view}

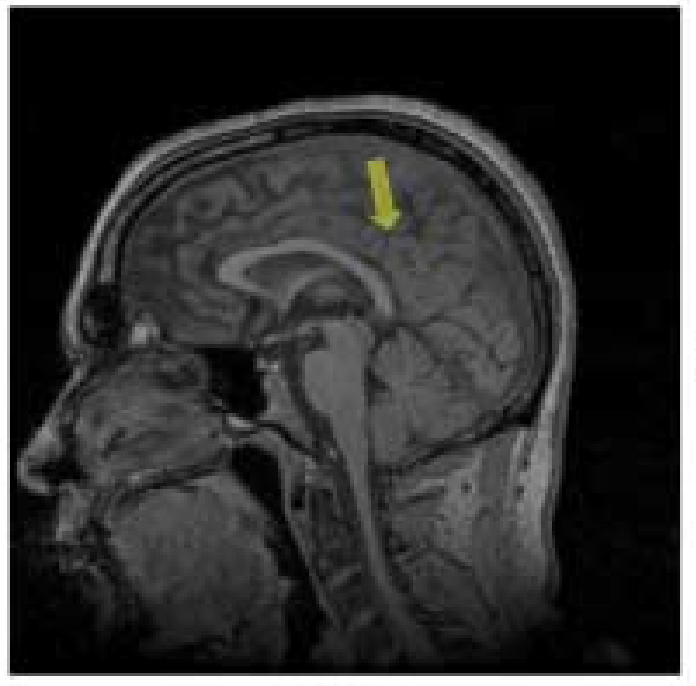

Sagittal view

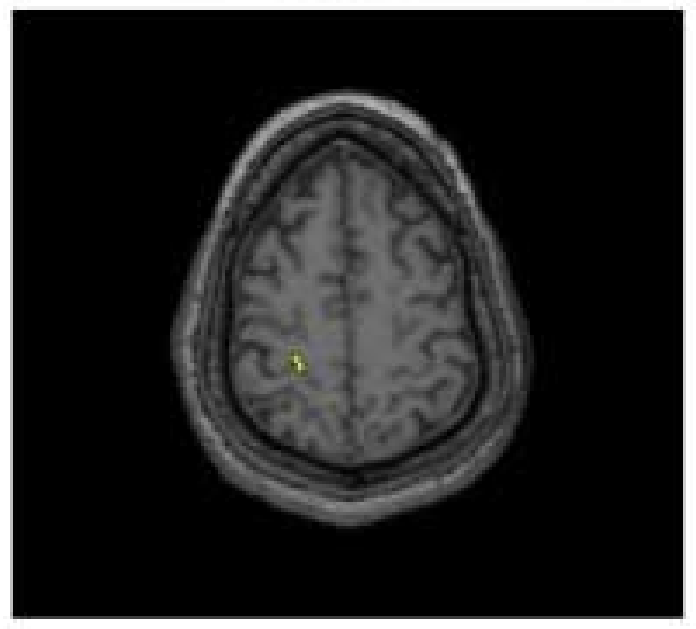

Superior view

Figure 3.12. Sectional views of the source localization results in a paraplegic patient, passive movement test. 

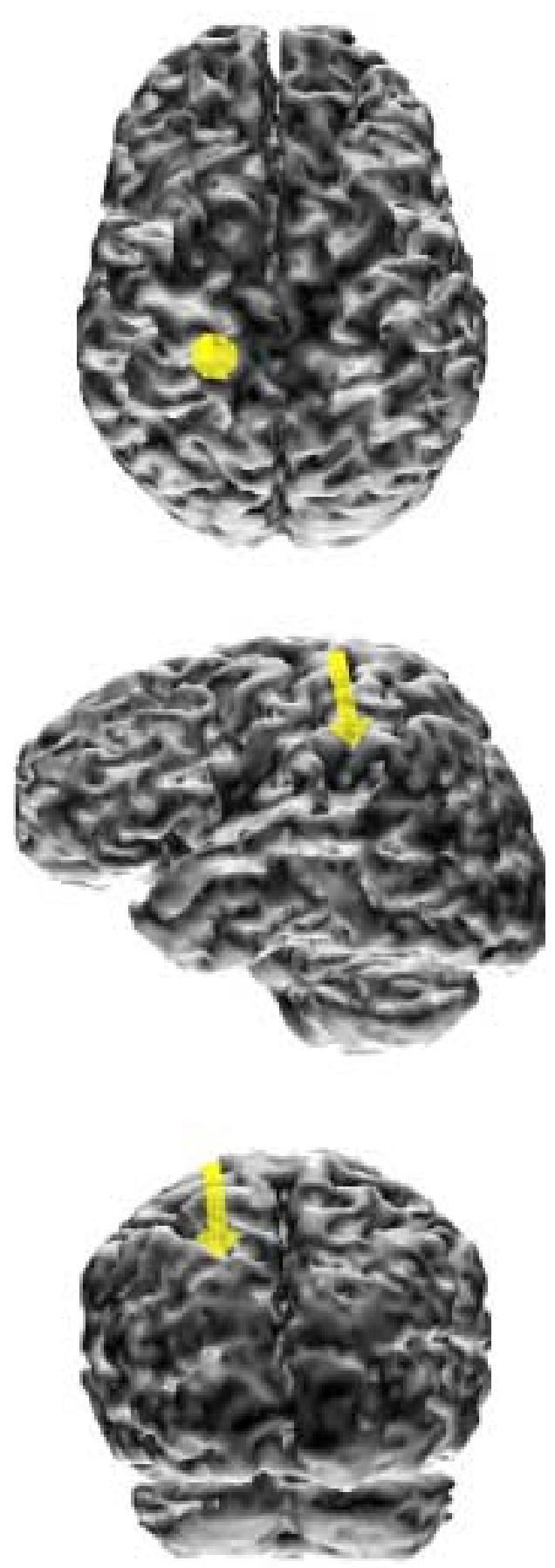

Figure 3.13. Source localization results with a segmented brain model - paraplegic patient, passive movement test. 
Table 3.13. Control subjects: Normalized coordinates and angle values in the source localization study.

\begin{tabular}{|c|c|c|c|c|c|c|c|c|c|c|c|c|c|}
\hline & \multirow[t]{2}{*}{ Controls } & \multicolumn{2}{|c|}{ LFSP } & \multicolumn{2}{|c|}{ LFPM } & \multicolumn{2}{|c|}{ RFSP } & \multicolumn{2}{|c|}{ RFPM } & \multicolumn{4}{|c|}{ Angle from the positive $y$-axis, degrees } \\
\hline & & abscissa & ordinate & abscissa & ordinate & abscissa & abscissa & abscissa & abscissa & LFSP & LFPM & RFSP & RFPM \\
\hline 1 & B. M. & & & & & -0.34 & 0.341 & -0.383 & 0.301 & & & -44.9159 & -51.8362 \\
\hline 2 & D. I. & 0.307 & 0.072 & 0.428 & 0.06 & -0.352 & 0.054 & -0.245 & 0.029 & 76.8011 & 82.01989 & -81.2783 & -83.2495 \\
\hline 3 & G. A. & 0.416 & 0.045 & & & -0.34 & 0.048 & & & 83.8261 & & -81.9643 & \\
\hline 4 & M. A. & 0.277 & 0.111 & 0.438 & 0.093 & & & & & 68.1629 & 78.01249 & & \\
\hline 5 & S. L. & 0.257 & 0.111 & 0.205 & 0.199 & -0.396 & 0.105 & -0.379 & 0.056 & 66.6402 & 45.85086 & -75.1497 & -81.5949 \\
\hline 6 & V. A. & 0.468 & 0.159 & 0.392 & 0.152 & -0.262 & 0.038 & -0.303 & 0.081 & 71.2351 & 68.80594 & -81.7475 & -75.0333 \\
\hline 7 & B. A. & & & & & & & -0.338 & 0.252 & & & & -53.2932 \\
\hline 8 & W. J. F. & & & & & -0.12 & 0.173 & -0.248 & 0.159 & & & -34.7468 & -57.3349 \\
\hline 9 & S. D. & & & & & -0.269 & 0.275 & -0.308 & 0.256 & & & -44.3681 & -50.2677 \\
\hline 10 & K. D. & & & & & -0.186 & 0.266 & -0.352 & 0.195 & & & -34.9631 & -61.0145 \\
\hline & Mean & 0.345 & 0.0996 & 0.36575 & 0.126 & -0.28313 & 0.1625 & -0.3195 & 0.166125 & 73.3331 & 68.6723 & -59.8917 & -64.203 \\
\hline & Std deviation & 0.092171 & 0.043391 & 0.108972 & 0.061779 & 0.093077 & 0.118942 & 0.053503 & 0.101914 & 7.03666 & 16.18881 & 21.95458 & 13.6593 \\
\hline
\end{tabular}


Table 3.14. SCI patients: Normalized coordinates and angle values in the source localization study.

\begin{tabular}{|c|c|c|c|c|c|c|c|c|c|c|c|c|c|}
\hline \multirow{2}{*}{\multicolumn{2}{|c|}{ SCI }} & \multicolumn{2}{|c|}{ LFSP } & \multicolumn{2}{|c|}{ LFPM } & \multicolumn{2}{|c|}{ RFSP } & \multicolumn{2}{|c|}{ RFPM } & \multicolumn{4}{|c|}{ Angle from the positive $y$-axis, degrees } \\
\hline & & abscissa & ordinate & abscissa & ordinate & abscissa & ordinate & abscissa & ordinate & LFSP & LFPM & RFSP & RFPM \\
\hline 1 & B. T./para & 0.218 & -0.101 & 0.379 & -0.098 & -0.227 & 0.039 & -0.125 & 0.017 & 114.858 & 104.4977 & -80.2514 & -82.2553 \\
\hline 2 & B. D. /para & 0.28 & -0.149 & & & -0.277 & -0.073 & -0.315 & -0.145 & 118.019 & & -104.764 & -114.717 \\
\hline 3 & H. R. /para & & & & & -0.35 & -0.123 & -0.269 & -0.277 & & & -109.363 & -135.839 \\
\hline 5 & H. R. /para & 0.213 & -0.118 & 0.331 & -0.191 & -0.154 & -0.056 & -0.19 & -0.126 & 118.986 & 119.9866 & -109.983 & -123.551 \\
\hline 6 & J. R. /para & 0.178 & -0.31 & 0.513 & -0.123 & & & & & 150.136 & 103.4831 & & \\
\hline 7 & J. R. /tetra & & & 0.369 & -0.205 & -0.177 & -0.137 & -0.189 & -0.203 & & 119.0546 & -127.74 & -137.045 \\
\hline 8 & M. B. /para & & & & & -0.285 & -0.186 & -0.262 & -0.086 & & & -123.13 & -108.172 \\
\hline 11 & W. B. /para & 0.157 & -0.284 & 0.285 & -0.169 & -0.265 & -0.292 & -0.416 & -0.216 & 151.065 & 120.6672 & -137.775 & -117.44 \\
\hline 12 & W. J./tetra & & & 0.242 & -0.357 & -0.248 & -0.084 & -0.333 & -0.113 & & 145.8678 & -108.712 & -108.744 \\
\hline & Mean & 0.243571 & -0.18643 & 0.3555 & -0.2035 & -0.2898 & -0.1132 & -0.28822 & -0.141 & 127.755 & 119.5879 & -110.857 & -114.599 \\
\hline & Std deviation & 0.096745 & 0.092716 & 0.079742 & 0.108778 & 0.109419 & 0.086811 & 0.116725 & 0.08472 & 19.1936 & 15.5418 & 15.82115 & 16.90155 \\
\hline
\end{tabular}




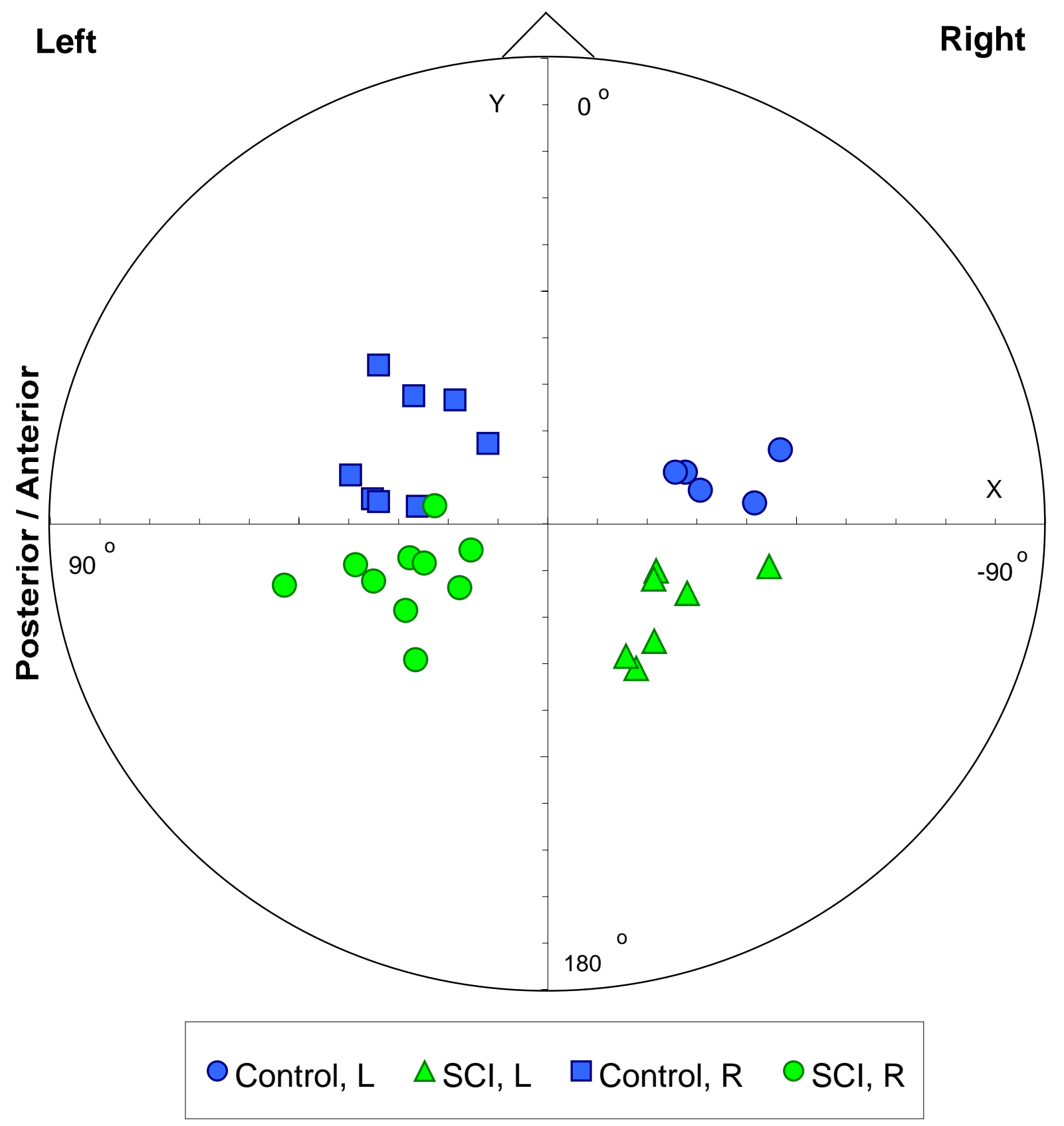

Figure 3.14. Dipole source localization in left and right finger self-paced movements. 


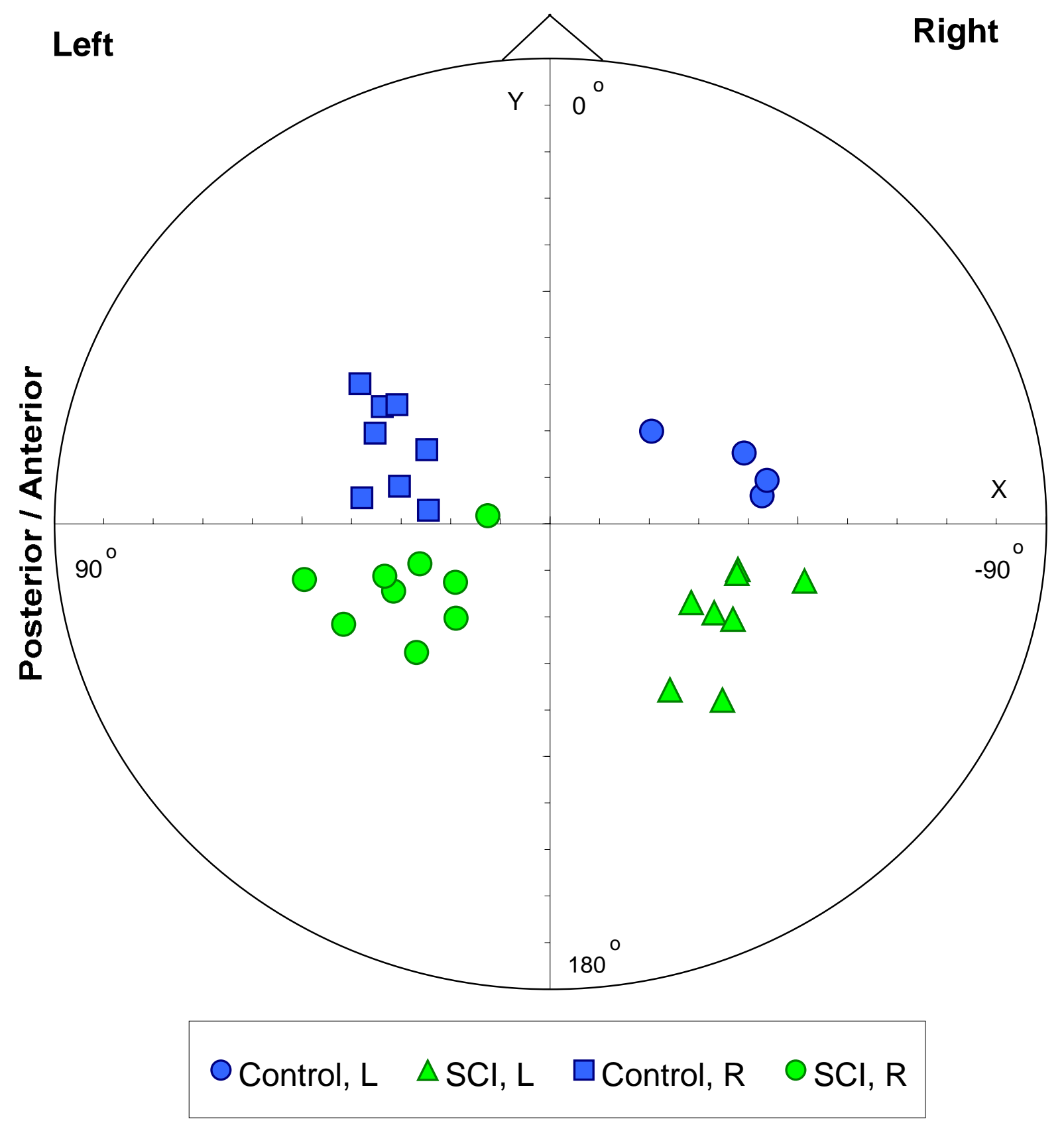

Figure 3.15. Dipole source localization in left and right finger passive movements. 
Table 3.15. Controls and SCI patients: $F$ test for the equality of two variances in source localization study.

\begin{tabular}{|lcccc|}
\hline & LFSP & RFSP & LFPM & RFPM \\
\hline Critical value: upper limit & $\mathrm{F}_{4,6,975}=6.23$ & $\mathrm{~F}_{7,9,975}=4.2$ & $\mathrm{~F}_{3,7,975}=5.89$ & $\mathrm{~F}_{7,8,975}=4.53$ \\
Critical value: lower limit & $\mathrm{F}_{4,6,025}=0.1087$ & $\mathrm{~F}_{7,9,025}=0.2066$ & $\mathrm{~F}_{3,7,025}=0.0684$ & $\mathrm{~F}_{7,8,025}=0.2041$ \\
Variance norm, $\mathrm{x}$ & & & & \\
Variance SCI, $\mathrm{x}$ & 0.008495 & 0.008663 & 0.011875 & 0.002863 \\
F statistic & 0.00936 & 0.011973 & 0.006359 & 0.013625 \\
Hypothesis Ho & 0.907676 & 0.72359 & 1.867461 & 0.210102 \\
& accepted & accepted & accepted & accepted \\
Variance norm, y & & & & \\
Variance SCI, y & 0.001883 & 0.014147 & 0.003817 & 0.010386 \\
F statistic & 0.008596 & 0.007536 & 0.011833 & 0.007178 \\
Hypothesis Ho & 0.219025 & 1.877231 & 0.322556 & 1.447079 \\
& accepted & accepted & accepted & accepted \\
Variance norm, angle & & & & \\
Variance SCI, angle & 49.51461 & 482.0037 & 262.0776 & 186.5764 \\
F statistic & 368.3949 & 250.3088 & 241.5476 & 285.6623 \\
Hypothesis Ho & 0.134406 & 1.925637 & 1.084994 & 0.653136 \\
\hline
\end{tabular}




\subsubsection{Source localization: the t-test}

The results of the $F$ test show that we can proceed to testing for the equality of means assuming equal variances. Table 3.16 shows the results of the hypothesis testing. It contains the critical values of the confidence interval for each test considered, the mean values, test statistics, $p$-values, and the test results with respect to the null hypothesis. It can be observed that in the two groups being compared, normal control subjects and SCI patients, the mean values of the spatial distribution of sources are not statistically different along the $\mathrm{x}$-axis in any of the four tests. The angles and y-components of the source coordinates, however, show strong correlation between condition and source location. This can be seen from the rejection of the null hypothesis for these tests as well as from the $p$-values being much less than 0.05 , the probability of a type I error.

\subsection{Discussion and Conclusion}

In order to achieve the goals set forth at the beginning of the project, the technology of high-resolution EEG co-registered with MRI was applied to non-invasively investigate the brain's movement control network in both SCI and normal subjects. A new device that triggers the acquisition computer in passive movement tests has been developed and successfully tested. The use of this device has increased the accuracy of measuring the beginning of each passive movement. This results in a higher signal-to-noise ratio (SNR) of the averaged EEG epochs and, as a consequence, the accuracy in localizing the activation. 
Table 3.16. Controls and SCI patients: $t$-test for the equality of means in source localization study.

\begin{tabular}{|lllll|}
\hline & \multicolumn{1}{c}{ LFSP } & \multicolumn{1}{c}{ RFSP } & \multicolumn{1}{c|}{ LFPM } & RFPM \\
\hline Critical value (equal variance) & $\mathrm{t}_{10,975}=2.22814$ & $\mathrm{t}_{16,975}=2.11990$ & $\mathrm{t}_{10,975}=2.22814$ & $\mathrm{t}_{15,975}=2.13145$ \\
Mean norm, $\mathrm{x}$ & & & & \\
Mean SCI, $\mathrm{x}$ & 0.345 & -0.28313 & 0.36575 & -0.3195 \\
Test statistic & 0.243571429 & -0.2898 & 0.3555 & -0.28822 \\
$p$-value & 1.824507358 & 0.137168 & 0.186979 & -0.69401 \\
Hypothesis Ho & 0.098052232 & 0.892609 & 0.855416 & 0.498281 \\
& accepted & accepted & accepted & accepted \\
Mean norm, y & & & & \\
Mean SCI, y & 0.0996 & 0.1625 & 0.126 & 0.166125 \\
Test statistic & -0.186428571 & -0.1132 & -0.2035 & -0.141 \\
$p$-value & 6.35369444 & 5.69161 & 5.541593 & 6.786134 \\
Hypothesis Ho & $8.31218 \mathrm{E}-05$ & $3.34 \mathrm{E}-05$ & 0.000247 & $6.14 \mathrm{E}-06$ \\
& rejected & rejected & rejected & rejected \\
Mean norm, angle & & & & \\
Mean SCI, angle & 73.33309607 & -59.8917 & 68.6723 & -64.203 \\
Test statistic & 127.7548983 & -110.857 & 119.5879 & -114.599 \\
$p$-value & -5.988933406 & 5.729464 & -5.28282 & 6.70279 \\
Hypothesis Ho & 0.000134074 & $3.1 \mathrm{E}-05$ & 0.000356 & $7.07 \mathrm{E}-06$ \\
\hline
\end{tabular}

The results of statistical analysis of the motor potential distribution data show strong correlation between the condition and location of the sites of activation in movement-related tasks. No statistically significant difference has been observed in the location of active areas along the medial-lateral direction in any two groups of subjects compared. However, the difference in the distribution of active sites along the anterior-posterior direction was found to be statistically significant in both voluntary and passive movement tests with the $p$-values much less than 0.05 . This difference was more pronounced in the tetraplegic group of patients than in the paraplegic group, as indicated by the corresponding $p$-values (Tables 3.9 and 3.10). 
These results were also corroborated using the dipole source analysis technique, which allowed localization of putative current sources in the brain responsible for the motor potential patterns observed in the EEG recordings. The DSA method makes use of the three-dimensional position of each EEG electrode overlaid on the subject's individual anatomy of the head derived from their MR images. Knowing the time course of each EEG channel and possessing a segmented individual model of the brain, we found current sources for each subject. The results of the statistical analysis confirmed the results of the motor potential localization study. A consistent posterior shift of current sources was found in the SCI group compared with the source locations in the normal control group. The $p$-values allow a numerical estimation of the degree of source relocations. Of the four movement-related tests performed, the maximal $p$-value was 0.000247 for the comparison of the source distributions along the anterior-posterior direction (Table 3.16). The lower $p$-values obtained in the DSA study demonstrate that this method not only confirms the results of the motor potential localization study, but also proves to be superior in showing the difference in EEG data.

An explanation of the posterior shift of motor potentials has been proposed by Green $[6,8]$. Because of the greater loss of axons in the primary motor area, the primary sensory cortex (S1) contribution to the generation of the MP is relatively increased, causing the origin of the potential to move behind the central sulcus. Peak MP latencies are longer than normal, suggestive of a change in pathway. This interpretation is based on the hypothesis that there is a relative sparing of the $\mathrm{S} 1$ outflow to the spinal cord after SCI. It is known that the S1 axons have a more medial and posterior course [27], which may render them less vulnerable to trauma.

Results of the passive movement tests show that moving a subject's finger also produces motor potentials, which are similar in location to those produced by voluntary movement. We 
believe that the MP, being part of the Movement-Related Cortical Potential complex, is generated by the sensorimotor cortex and assists in the generation of the contralateral discharge down the pyramidal tract. The "trigger" requires activation of M1 and the SMA, the latter being active only in voluntary movement (Figures 3.6, 3.7, 3.10 and 3.11). This may indicate that activation of the SMA may be the triggering mechanism for the generation of active movement.

The conclusions of this project are based on the results of statistical analysis of data obtained from samples that are finite and not, strictly speaking, heterogeneous, which is typical for a cross-sectional study. It should also be noted that since there are many variables involved, such as age, gender, ethnic origin, time from injury, and variations in individual anatomy and physiology, the interpretation of the results should be made from the probabilistic standpoint, thus emphasizing the idea that SCI patients as a group have shown consistent posterior relocation of motor potentials.

Future work can involve pursuits to make localization of current sources descriptive, in which case the co-planar stereotaxic atlas of the human brain [28] can be used. This would allow identification of brain regions of interest, in this context the regions where current sources are localized.

The results of the project can contribute to the understanding of how the brain reorganizes itself after SCI, enabling maximum utilization of the surviving connections, and improve patient recovery. Also, understanding the neuronal activity and its topography in the brain is important in view of recent success in experiments with owl monkeys. Inputs from invasively implanted EEG electrodes were processed and enabled the monkeys to control prosthetic devices [9]. This suggests that EEG may someday be used to produce an interface between the human brain and computer-driven prostheses and external devices. 


\section{LIST OF REFERENCES}




\section{LIST OF REFERENCES}

1. Stover SL, Delisa JA, Whiteneck GG. Spinal Cord Injury: Clinical Outcomes from the Model Systems. New York: Aspen Publishers, Inc., 1995. ISBN: 0834206978.

2. Tortora GJ, Grabowski SR. Principles of anatomy and physiology. $8^{\text {th }}$ ed. New York, NY: HarperCollins Publishers Inc., 1996.

3. Ono M, Kubik S, Abernathey CD. Atlas of the cerebral sulci. New York: Thieme Medical Publishers, 1990:36-41.

4. Nii Y, Uematsu S, Lesser RP, Gordon B. Does the central sulcus divide motor and sensory functions? Neurology 1996; 46:360-367.

5. Duffau H, Sichez JP, Lehericy S. Intraoperative unmasking of brain redundant motor sites during resection of a precentral angioma: evidence using direct cortical stimulation. Ann Neurol 2000; 47:132-135.

6. Green JB, Sora E, Bialy Y, Ricamato A, Thatcher RW. Cortical motor reorganization after paraplegia: an EEG study. Neurology 1999; 53(4):736-743.

7. Tarkka IM, Hallett M. Topography of scalp-recorded motor potentials in human finger movements. J Clin Neurophysiol 1991; 8(3):331-341.

8. Green JB, Sora E, Bialy Y, Ricamato A, Thatcher RW. Cortical sensorimotor reorganization after spinal cord injury: an electroencephalographic study. Neurology 1998; 50(4):1115-1121.

9. Wessberg J, Stambaugh CR, Kralik JD, Beck PD, Laubach M, Chapin JK, Kim J, Biggs SJ, Srinivasan MA, Nicolelis MAL. Real-time prediction of hand trajectory by ensembles of cortical neurons in primates. Nature 2000; 408:361-365.

10. Schmidt EM. Single neuron recording from motor cortex as a possible source of signals for control of external devices. Ann Biomed Eng 1980; 8:339-349.

11. Kornhuber HH und Deecke L. Hirnpotentialanderungen bei Willkurbewegungen und passiven Bewegungen des Mensehen: Bereitschaftspotential und reafferente Potentiale. Pflugers Arch Ges Physiol 1965; 284:1-17.

12. Neshige R, Luders H, Shibasaki H. Recording of movement-related potentials from scalp and cortex in man. Brain 1988; 111 (Pt 3):719-736.

13. Toro C, Matsumoto J, Deuschl G, Roth BJ, Hallett M. Source analysis of scalp-recorded movement-related electrical potentials. Electroencephalogr Clin Neurophysiol 1993; 86(3):167175. 
14. Cheyne D, Weinberg H. Neuromagnetic fields accompanying unilateral finger movements: pre-movement and movement-evoked fields. Exp Brain Res 1989; 78(3):604-612.

15. Kristeva R, Cheyne D, Deecke L. Neuromagnetic fields accompanying unilateral and bilateral voluntary movements: topography and analysis of cortical sources. Electroencephalogr Clin Neurophysiol 1991; 81(4):284-298.

16. Turner JA, Lee JS, Martinez O, Medlin AL, Schandler SL, Cohen MJ. Somatotopy of the motor cortex after long-term spinal cord injury or amputation. IEEE Trans Neural Syst Rehabil Eng 2001; 9(2):154-160.

17. Shibasaki H, Barrett G, Halliday E, Halliday AM. Cortical potentials following voluntary and passive finger movements. Electroenceph Clin Neurophysiol 1980; 50(3-4):201-213.

18. Weiller C, Juptner M, Fellows S, Rijntjes M, Leonhardt G, Kiebel S, Muller S, Diener HC, Thilmann AF. Brain representation of active and passive movements. Neuroimage 1996; 4(2):105-110.

19. Weiller C, Leonhardt G, Rijntjes M, Dettmers Ch, Muller S, Juptner M. Early sensory reorganization predicts recovery of lost motor function after stroke - a clinical PET study. Neuroimage 1997; 5:s28.

20. Alary F, Doyon B, Loubinoux I, Carel C, Boulanouar K, Ranjeva JP, Celsis P, Chollet F. Event-related potentials elicited by passive movements in humans: characterization, source analysis, and comparison to fMRI. Neuroimage 1998; 8(4):377-390.

21. Nelles G, Spiekramann G, Jueptner M, Leonhardt G, Muller S, Gerhard H, Diener HC. Evolution of functional reorganization in hemiplegic stroke: a serial positron emission tomographic activation study. Ann Neurol 1999; 46(6):901-909.

22. Mima T, Terada K. Discrimination of brain structures related to "pure" motor and sensory feedback component of voluntary finger movement. Electroenceph Clin Neurophysiol 1995; 4(s):s97.

23. Philips Electronics N.V. Current reconstruction and imaging. EEG software version 3.0. El Paso, TX: Neuroscan, 1998.

24. Fuchs M, Drenckhahn R, Wischman HA, Wagner M. An improved boundary element model for realistic volume conductor modeling. IEEE Trans Biomed Eng 1998 Aug;45(8):980-997.

25. Fuchs M, Wagner M, Kastner J. Boundary element method volume conductor models for EEG source reconstruction. Clin Neurophysiol 2001; 112(8):1400-1407.

26. Jackson J.D. Classical Electrodynamics. $3^{\text {rd }}$ ed. New York, NY: John Wiley \& Sons, 1998. 
27. Ghez C. The control of movement. In: Kandel ER, Schwartz JH, Lessel TM, eds. Principles of neural science. $3^{\text {rd }}$ ed. Norwalk, CT: Appleton and Lange, 1991:534-547.

28. Talairach J, Tournoux P. Co-Planar Stereotaxic Atlas of the Human Brain. New York: Thieme Medical Publishers, 1988. 
APPENDIX 


\section{APPENDIX}

// Coordinates.java

// The program calculates the coordinates of electrode(s) of interest import javax.swing.JOptionPane;

import java.text.DecimalFormat;

public class Coordinates \{

static double $\mathrm{x} \_$PAR, $\mathrm{x}$ _PAL, y_Nas;

static double tri_center_x, tri_center_y;

public static void main( String args[] )

\{

DecimalFormat precision3 = new DecimalFormat( "0.000" );

String s1 = JOptionPane.showInputDialog(

"Enter the x-coordinate of PAR" );

String s2 = JOptionPane.showInputDialog(

"Enter the X-coordinate of PAL" );

String s3 = JOptionPane.showInputDialog(

"Enter the y-coordinate of Nasion" );

x_PAR $=$ Double.parseDouble( s1 );

$\mathrm{x} \_\mathrm{PAL}=$ Double.parseDouble( s2 );

y_Nas = Double.parseDouble( $\mathrm{s} 3$ );

String s4 = JOptionPane.showInputDialog(

"How many electrodes are to be enetered $(1,2,3)$ ?" );

int num = Integer.parseInt ( s4 );

switch( num ) \{

case 1:

String s5 = JOptionPane.showInputDialog(

"Enter the electrode x-coordinate" );

String s6 = JOptionPane.showInputDialog(

"Enter the electrode y-coordinate" );

double x1_1 = Double.parseDouble( s5 );

double y1_1 = Double.parseDouble( s6 );

double norm_X_1 = normalizeX $\left(\mathrm{x} 1 \_1\right)$;

double norm_Y_1 = normalizeY $\left(\mathrm{y}_{-} 1\right.$ );

JOptionPane.showMessageDialog(

null,"The normalized coordinates for one electrode are ("+ precision3.format(norm_X_1) + "; " + 
precision3.format( norm_Y_1 ) + ")",

"Results", JOptionPane.INFORMATION_MESSAGE );

break;

case 2:

String s7 = JOptionPane.showInputDialog(

"Enter the first electrode x-coordinate" );

String s8 = JOptionPane.showInputDialog(

"Enter the first electrode y-coordinate" );

String s9 = JOptionPane.showInputDialog(

"Enter the second electrode x-coordinate" );

String s10 = JOptionPane.showInputDialog (

"Enter the second electrode y-coordinate" );

double x2_1 = Double.parseDouble( s7 );

double y2_1 = Double.parseDouble( s8);

double x2_2 = Double.parseDouble( s9);

double y2_2 = Double.parseDouble( s10);

double norm_X_two $=$ normalizeX_two $\left(\mathrm{x} 2 \_1, \mathrm{x} 2 \_2\right)$;

double norm_Y_two $=$ normalizeY_two $\left(y 2 \_1, y 2 \_2\right)$;

JOptionPane.showMessageDialog(

null,"The normalized coordinates for two electrodes are ("+

precision3.format (norm_X_two ) + "; " +

precision3.format ( norm_Y_two ) + ")",

"Results", JOptionPane.INFORMATION_MESSAGE );

break;

case 3:

String s11 = JOptionPane. showInputDialog(

"Enter the first electrode x-coordinate" );

String s12 = JOptionPane.showInputDialog(

"Enter the first electrode y-coordinate" );

String s13 = JOptionPane. showInputDialog(

"Enter the second electrode x-coordinate" );

String s14 = JOptionPane.showInputDialog(

"Enter the second electrode y-coordinate" );

String s15 = JOptionPane.showInputDialog(

"Enter the third electrode x-coordinate" );

String s16 = JOptionPane. showInputDialog(

"Enter the third electrode y-coordinate" );

double x3_1 = Double.parseDouble( s11 );

double y3_1 = Double.parseDouble( s12);

double x3_2 = Double.parseDouble( s13);

double y3_2 = Double.parseDouble( s14);

double x3_3 = Double.parseDouble( s15);

double y3_3= Double.parseDouble( s16 ); 


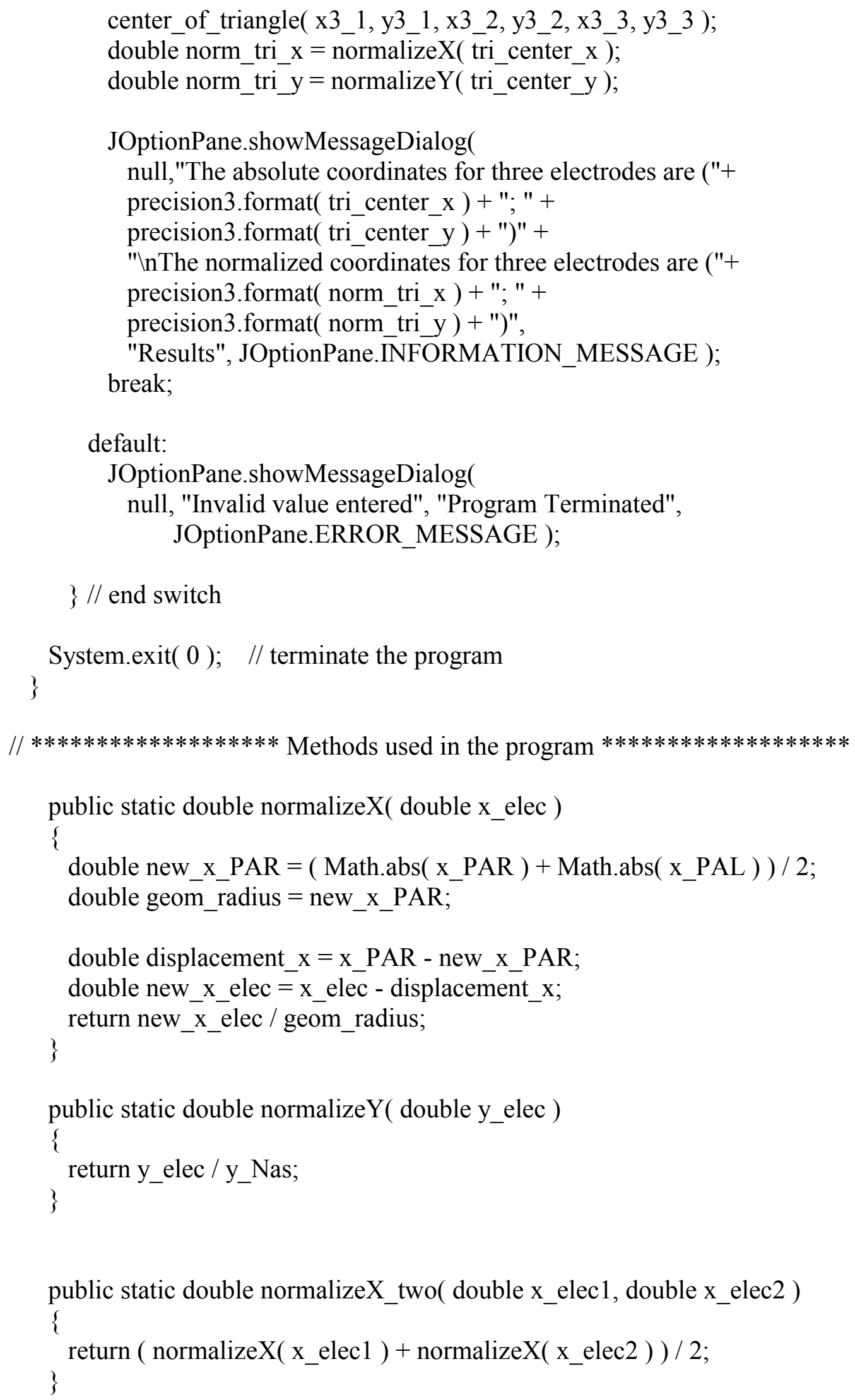




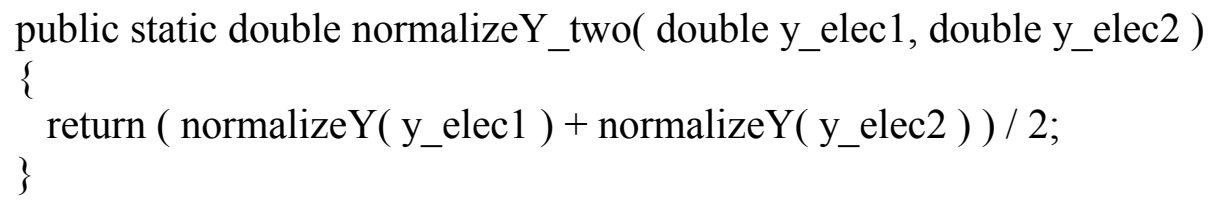




\section{VITA}

Leonid I. Rozhkov was born in the USSR in 1973. He received the degree of Engineer with the specialization in Biotechnical and Medical Apparatuses and Systems from Ternopol Instrument Making Institute, Ternopol, Ukraine. He joined the graduate program in Biomedical Engineering in August 1998 at The University of Tennessee, Memphis and The University of Memphis. He worked for Dr. Herbert D. Zeman from August 1998 till August 1999. In August 1999, he started working for Dr. Joseph B. Green in the Spinal Cord Injury Research Lab at the Memphis VA Medical Center. 